\title{
GEOMETRIC CONSTRUCTION OF MODULAR FUNCTORS FROM CONFORMAL FIELD THEORY
}

\author{
JØRGEN ELLEGAARD ANDERSEN AND KENJI UENO
}

\begin{abstract}
We give a geometric construct of a modular functor for any simple Lie-algebra and any level by twisting the constructions in [16 and [19] by a certain fractional power of the abelian theory first considered in 13 , and further studied in [2].
\end{abstract}

\section{Contents}

1. Introduction

2. The axioms for a modular functor

3. Teichmüller space and families of pointed Riemann Surfaces with formal neighbourhoods

4. The space of vacua associated to a labeled marked Riemann Surface

4.1. Affine Lie algebras and integrable highest weight modules

4.2. The Segal-Sugawara construction

4.3. The space of vacua

4.4. Propagation of vacua

4.5. Change of formal neighbourhoods

4.6. The definition of the space of vacua associated to a labeled marked Riemann surface.

5. The bundle of vacua over Teichmüller space

5.1. Definition of the sheaf of vacua

5.2. Properties of the sheaf of vacua.

5.3. The definition of the bundle of vacua over Teichmüller space

6. The connection in the bundle of vacua over Teichmüller space

6.1. Twisted first order differential operators acting on the sheaf of vacua

6.2. The projectively flat connections on the on the bundle of vacua

6.3. The definition of the connection in the bundle of vacua over Teichmüller space

7. Definition of the space of abelian vacua associated to a Riemann surface.

7.1. Fermion Fock space

This research was conducted partly by the first author for the Clay Mathematics Institute at University of California, Berkeley and for MaPhySto - A Network for Mathematical Physics and Stochastics, funded by The Danish National Research Foundation. The second author is partially supported by Grant in Aid for Scientific Research NO. 14102001 of JSPS. 
7.2. Abelian Vacua

7.3. The space of abelian vacua associated to a Riemann surface

8. Definition of the line bundle of abelian vacua over Teichmüller space

8.1. Sheaf of abelian vacua

8.2. The line bundle of abelian vacua over Teichmüller space

9. The connection in the line bundle of abelian vacua over Teichmüller space.

10. The preferred non-vanishing section of the bundle of abelian vacua.

11. The geometric construction of the modular functor.

12. Sheaf of vacua and gluing.

13. Verification of the axioms

14. Appendix. Families of stable curves and glueing

14.1. Stable curves

14.2. Sheaf of vacua for a family of pointed stable curves

14.3. The sheaf of abelian vacua associated to families of pointed stable curves

14.4. The glueing of vacua construction

References

\section{INTRODUCTION}

This is the second paper in a series of three papers ([2] and [3]) in which we provide a geometric construction of modular functors and topological quantum field theories from conformal field theory building on the constructions in [16] and [19] and [13. In this paper we provide the geometric construction of a modular functor $V_{\ell}^{\mathfrak{g}}$ for each simple Lie algebra $\mathfrak{g}$ and a positive integer $\ell$ (the level). In our third paper 3 in this series we give an explicit isomorphism of the modular functor underlying the Reshetikhin-Turaev TQFT for $U_{q}(\mathrm{sl}(n))$ and the one constructed in this paper for the Lie algebra $\mathrm{sI}(n)$. This uses the Skein theory approach to the Reshetikhin-Turaev TQFT of Blanchet, Habegger, Masbaum and Vogel [7, [8] and [6]. In particular we use Blanchet's [6] constructions of the Hecke-category and its associated modular tensor categories. This construction is really a generation of the BHMV-construction of the $U_{q}\left(s l_{2}(\mathbb{C})\right)$-Reshetikhin-Turaev TQFT 8$]$ to the $U_{q}\left(s l_{n}(\mathbb{C})\right)$-case. As a consequence of this, we construct a duality and a unitary structure on our modular functor in the case of $\mathfrak{g}=\mathrm{sl}(n)$.

By a very general construction any modular functor with duality induces a topological quantum field theory in dimension $2+1$ by the work of Kontsevich [14] and Walker [20] and also Grove [11. By applying this to the modular functor $V_{\ell}^{\mathfrak{g}}$, for $\mathfrak{g}=\mathrm{sl}(n)$, we get a TQFT for each $\ell$. We also prove in [3] that this TQFT is isomorphic to the Reshetikhin-Turaev TQFT for $U_{q}(\mathrm{sI}(n))$ at level $\ell$.

Let us now describe our construction. Fix a simple Lie algebra $\mathfrak{g}$ and normalize the invariant inner product on it by requiring the highest root to have length squared equal to 2 . Let $\ell$ be a 
positive integer and consider the finite (label) set $P_{\ell}$ of integrable highest weight representations at level $\ell$ of the affine Lie algebra of $\mathfrak{g}$. By the usual highest weight vector representations, this finite set $P_{\ell}$ is naturally identified with a subset of the dominant integrable weights of $\mathfrak{g}$ (see formula (6) ).

The main idea is to construct a modular functor $V_{\ell}^{\mathfrak{g}}$ by associating to each labeled marked surface the space of vacua using the given labels for the Lie algebra $\mathfrak{g}$ at level $\ell$ for some complex structure on the marked surface, as defined in [16] [18] and [19. In order to make this construction independent of the complex structure it must be understood in terms of bundles with connections over the hole of Teichmüller space of the surface, relying on parallel transport to provide the required identifications between the different spaces of vacua. The consistencies of these identifications translates to flatness requirements on these connections. However, the sheaf of vacua construction in [16] and [19] gives a bundles with a connections, which is only projectively flat, over Teichmüller space of the surface. By tensoring this bundle with a line bundle with a connection with the opposite curvature, we get a flat bundle over Teichmüller space and the vector space we associate to the labeled marked surface is the vector space of covariant constant sections of this resulting bundle. This line bundle is constructed as a fractional power of a certain rank 1 abelian sheaf of vacua, which we considered in the first paper in this series 2] from the same point of view as [16] and [19. The extraction of this fractional power brings in central extensions of mapping classes as the natural morphisms on which the resulting functor is defined.

The construction and properties of this flat bundle primarily rely on the complex algebraic constructions and results of [16] and [19] on the the sheaf of vacua construction yielding a conformal field theory for each simple Lie algebra $\mathfrak{g}$ and level $\ell$. For the 1-dimensional correction theory, we draw on the work [2, which in turn relies on [13].

The definition of the functor $V_{\ell}^{\mathfrak{g}}$ requires only considerations of smooth families of Riemann surfaces (with some extra structure which is specified in section 3) over smooth complex manifolds. This is the setting for section 3 through to section 11 However, in order to define the glueing isomorphism, which a modular functor is required to have, we need to discuss certain very simple families of stable curves, which contains so call nodal curves. These are described and considered in section 12 and in the Appendix to this paper.

The paper is organized as follows. In section 2 we give the axioms for a modular functor. We introduce the notion of a marked surface, which is a closed smooth oriented surface, with a finite subset of points with projective tangent vectors and a Lagrangian subspace of the first integer homology of the surface. These form a category on which there is the operation of disjoint union and the operation of orientation reversal. There is also the process of glueing on this category. If we have a finite set, we can label the finite set of points on a marked surface by elements from this finite label set and get the category of labeled marked surfaces. A modular functor based on some finite label set, is a functor from the category of labeled marked surfaces to the category of finite dimensional complex vector spaces, which takes the disjoint union operation to the tensor product 
operation and which takes the glueing process to a certain direct sum construction, such that some compatibility holds, as described in details in Definition 2.11. A modular functor is said to be with duality if further the operation of orientation reversal is taken to the operation of taking the dual vector space.

In sections 3 to 6 we describe in detail how any simple Lie algebra and a level $\ell$, via the sheaf of vacua constructions in [19] yields a holomorphic vector bundles with a projectively flat connection over Teichmüller spaces of pointed surfaces equipped with symplectic basis of the first homology. In sections 7 to 10 we describe in detail how the abelian sheaf of vacua constructions in [2] yields a holomorphic line bundles with a projectively flat connection and a preferred non-vanishing section over Teichmüller spaces of pointed surfaces equipped with a symplectic basis of the first homology.

In section 11 we describe our global geometric construction of a modular functor for any simple Lie algebra and a level $\ell$. Theorem 11.1 and 11.2 summarizes the constructions from sections 3 to 10 . The preferred section of the abelian theory allows us to construct a certain fractional power of this line bundle as stated in Theorem 11.3, which we tensor onto this holomorphic vector bundle, so as to obtain a holomorphic vector bundle with a flat connection over Teichmüller space. The modular functor is then defined (Definition 11.3) by taking covariant constant sections of this flat bundle. The section ends with the construction of the disjoint union isomorphism. The glueing isomorphism is constructed in section 12, where we also prove the needed properties of glueing.

In section 13 we establish all the axioms of a modular functor is satisfied based on the main results of the preceding sections.

We have included an Appendix, which recalls the nessessary definitions regarding nodal curves, families of stable curves and the glueing construction.

\section{THE AXIOMS FOR A MODULAR FUNCTOR}

We shall in this section give the axioms for a modular functor. These are due to G. Segal and appeared first in [15]. We present them here in a topological form, which is due to K. Walker [20]. See also [1]. We note that similar, but different, axioms for a modular functor are given in [17] and in [5]. It is however not clear if these definitions of a modular functor is equivalent to ours.

Let us start by fixing a bit of notation. By a closed surface we mean a smooth real two dimensional manifold. For a closed oriented surface $\Sigma$ of genus $g$ we have the non-degenerate skew-symmetric intersection pairing

$$
(\cdot, \cdot): H_{1}(\Sigma, \mathbb{Z}) \times H_{1}(\Sigma, \mathbb{Z}) \rightarrow \mathbb{Z}
$$

Suppose $\Sigma$ is connected. In this case a Lagrangian subspace $L \subset H_{1}(\Sigma, \mathbb{Z})$ is by definition a subspace, which is maximally isotropic with respect to the intersection pairing. - A $\mathbb{Z}$-basis $(\vec{\alpha}, \vec{\beta})=$ $\left(\alpha_{1}, \ldots, \alpha_{g}, \beta_{1}, \ldots \beta_{g}\right)$ for $H_{1}(\Sigma, \mathbb{Z})$ is called a symplectic basis if

$$
\left(\alpha_{i}, \beta_{j}\right)=\delta_{i j}, \quad\left(\alpha_{i}, \alpha_{j}\right)=\left(\beta_{i}, \beta_{j}\right)=0,
$$


for all $i, j=1, \ldots, g$.

If $\Sigma$ is not connected, then $H_{1}(\Sigma, \mathbb{Z})=\oplus_{i} H_{1}\left(\Sigma_{i}, \mathbb{Z}\right)$, where $\Sigma_{i}$ are the connected components of $\Sigma$. By definition a Lagrangian subspace is in this paper a subspace of the form $L=\oplus_{i} L_{i}$, where $L_{i} \subset H_{1}\left(\Sigma_{i}, \mathbb{Z}\right)$ is Lagrangian. Likewise a symplectic basis for $H_{1}(\Sigma, \mathbb{Z})$ is a $\mathbb{Z}$-basis of the form $\left(\left(\vec{\alpha}^{i}, \vec{\beta}^{i}\right)\right)$, where $\left(\vec{\alpha}^{i}, \vec{\beta}^{i}\right)$ is a symplectic basis for $H_{1}\left(\Sigma_{i}, \mathbb{Z}\right)$.

For any real vector space $V$, we define $P V=(V-\{0\}) / \mathbb{R}_{+}$.

Definition 2.1. A pointed surface $(\Sigma, P)$ is an oriented closed surface $\Sigma$ with a finite set $P \subset \Sigma$ of points. A pointed surface is called stable if the Euler characteristic of each component of the complement of the points $P$ is negative. A pointed surface is called saturated if each component of $\Sigma$ contains at least one point from $P$.

Definition 2.2. A morphism of pointed surfaces $f:\left(\Sigma_{1}, P_{1}\right) \rightarrow\left(\Sigma_{2}, P_{2}\right)$ is an isotopy class of orientation preserving diffeomorphisms which maps $P_{1}$ to $P_{2}$. Here the isotopy is required not to change the induced map of the first order Jet at $P_{1}$ to the first order Jet at $P_{2}$.

Definition 2.3. A marked surface $\boldsymbol{\Sigma}=(\Sigma, P, V, L)$ is an oriented closed smooth surface $\Sigma$ with a finite subset $P \subset \Sigma$ of points with projective tangent vectors $V \in \sqcup_{p \in P} P T_{p} \Sigma$ and a Lagrangian subspace $L \subset H_{1}(\Sigma, \mathbb{Z})$.

Remark 2.1. The notions of stable and saturated marked surfaces are defined just like for pointed surfaces.

Definition 2.4. A morphism $\mathbf{f}: \boldsymbol{\Sigma}_{1} \rightarrow \boldsymbol{\Sigma}_{2}$ of marked surfaces $\boldsymbol{\Sigma}_{i}=\left(\Sigma_{i}, P_{i}, V_{i}, L_{i}\right)$ is an isotopy class of orientation preserving diffeomorphisms $f: \Sigma_{1} \rightarrow \Sigma_{2}$ that maps $\left(P_{1}, V_{1}\right)$ to $\left(P_{2}, V_{2}\right)$ together with an integer $s$. Hence we write $\mathbf{f}=(f, s)$.

Remark 2.2. Any marked surface has an underlying pointed surface, but a morphism of marked surfaces does not quit induce a morphism of pointed surfaces, since we only require that the isotopies preserve the induced maps on the projective tangent spaces.

Let $\sigma$ be Wall's signature cocycle for triples of Lagrangian subspaces of $H_{1}(\Sigma, \mathbb{R})$ (See [21).

Definition 2.5. Let $\mathbf{f}_{1}=\left(f_{1}, s_{1}\right): \boldsymbol{\Sigma}_{1} \rightarrow \boldsymbol{\Sigma}_{2}$ and $\mathbf{f}_{2}=\left(f_{2}, s_{2}\right): \boldsymbol{\Sigma}_{2} \rightarrow \boldsymbol{\Sigma}_{3}$ be morphisms of marked surfaces $\boldsymbol{\Sigma}_{i}=\left(\Sigma_{i}, P_{i}, V_{i}, L_{i}\right)$ then the composition of $\mathbf{f}_{1}$ and $\mathbf{f}_{2}$ is

$$
\mathbf{f}_{2} \mathbf{f}_{1}=\left(f_{2} f_{1}, s_{2}+s_{1}-\sigma\left(\left(f_{2} f_{1}\right)_{*} L_{1}, f_{2 *} L_{2}, L_{3}\right)\right) .
$$

With the objects being marked surfaces and the morphism and their composition being defined as in the above definition, we have constructed the category of marked surfaces.

The mapping class group $\Gamma(\boldsymbol{\Sigma})$ of a marked surface $\boldsymbol{\Sigma}=(\Sigma, L)$ is the group of automorphisms of $\boldsymbol{\Sigma}$. One can prove that $\Gamma(\boldsymbol{\Sigma})$ is a central extension of the mapping class group $\Gamma(\Sigma)$ of the surface $\Sigma$ defined by the 2-cocycle $c: \Gamma(\boldsymbol{\Sigma}) \rightarrow \mathbb{Z}, c\left(f_{1}, f_{2}\right)=\sigma\left(\left(f_{1} f_{2}\right)_{*} L, f_{1 *} L, L\right)$. One can also prove that 
this cocycle is equivalent to the cocycle obtained by considering two-framings on mapping cylinders (see [4] and [1]).

Notice also that for any morphism $(f, s): \boldsymbol{\Sigma}_{1} \rightarrow \boldsymbol{\Sigma}_{2}$, one can factor

$$
\begin{aligned}
(f, s) & =\left(\left(\mathrm{Id}, s^{\prime}\right): \boldsymbol{\Sigma}_{2} \rightarrow \boldsymbol{\Sigma}_{2}\right) \circ\left(f, s-s^{\prime}\right) \\
& =\left(f, s-s^{\prime}\right) \circ\left(\left(\mathrm{Id}, s^{\prime}\right): \boldsymbol{\Sigma}_{1} \rightarrow \boldsymbol{\Sigma}_{1}\right) .
\end{aligned}
$$

In particular $(\mathrm{Id}, s): \boldsymbol{\Sigma} \rightarrow \boldsymbol{\Sigma}$ is $(\mathrm{Id}, 1)^{s}$.

Definition 2.6. The operation of disjoint union of marked surfaces is

$$
\left(\Sigma_{1}, P_{1}, V_{1}, L_{1}\right) \sqcup\left(\Sigma_{2}, P_{2}, V_{2}, L_{2}\right)=\left(\Sigma_{1} \sqcup \Sigma_{2}, P_{1} \sqcup P_{2}, V_{1} \sqcup V_{2}, L_{1} \oplus L_{2}\right) .
$$

Morphisms on disjoint unions are accordingly $\left(f_{1}, s_{1}\right) \sqcup\left(f_{2}, s_{2}\right)=\left(f_{1} \sqcup f_{2}, s_{1}+s_{2}\right)$.

We see that disjoint union is an operation on the category of marked surfaces.

Definition 2.7. Let $\boldsymbol{\Sigma}$ be a marked surface. We denote by $-\boldsymbol{\Sigma}$ the marked surface obtained from $\boldsymbol{\Sigma}$ by the operation of reversal of the orientation. For a morphism $\mathbf{f}=(f, s): \boldsymbol{\Sigma}_{1} \rightarrow \boldsymbol{\Sigma}_{2}$ we let the orientation reversed morphism be given by $-\mathbf{f}=(f,-s):-\boldsymbol{\Sigma}_{1} \rightarrow-\boldsymbol{\Sigma}_{2}$.

We also see that orientation reversal is an operation on the category of marked surfaces. Let us now consider glueing of marked surfaces.

Let $\left(\Sigma,\left\{p_{-}, p_{+}\right\} \sqcup P,\left\{v_{-}, v_{+}\right\} \sqcup V, L\right)$ be a marked surface, where we have selected an ordered pair of marked points with projective tangent vectors $\left(\left(p_{-}, v_{-}\right),\left(p_{+}, v_{+}\right)\right)$, at which we will perform the glueing.

Let $c: P\left(T_{p_{-}} \Sigma\right) \rightarrow P\left(T_{p_{+}} \Sigma\right)$ be an orientation reversing projective linear isomorphism such that $c\left(v_{-}\right)=v_{+}$. Such a $c$ is called a glueing map for $\Sigma$. Let $\tilde{\Sigma}$ be the oriented surface with boundary obtained from $\Sigma$ by blowing up $p_{-}$and $p_{+}$, i.e.

$$
\tilde{\Sigma}=\left(\Sigma-\left\{p_{-}, p_{+}\right\}\right) \sqcup P\left(T_{p_{-}} \Sigma\right) \sqcup P\left(T_{p_{+}} \Sigma\right),
$$

with the natural smooth structure induced from $\Sigma$. Let now $\Sigma_{c}$ be the closed oriented surface obtained from $\tilde{\Sigma}$ by using $c$ to glue the boundary components of $\tilde{\Sigma}$. We call $\Sigma_{c}$ the glueing of $\Sigma$ at the ordered pair $\left(\left(p_{-}, v_{-}\right),\left(p_{+}, v_{+}\right)\right)$with respect to $c$.

Let now $\Sigma^{\prime}$ be the topological space obtained from $\Sigma$ by identifying $p_{-}$and $p_{+}$. We then have natural continuous maps $q: \Sigma_{c} \rightarrow \Sigma^{\prime}$ and $n: \Sigma \rightarrow \Sigma^{\prime}$. On the first homology group $n$ induces an injection and $q$ a surjection, so we can define a Lagrangian subspace $L_{c} \subset H_{1}\left(\Sigma_{c}, \mathbb{Z}\right)$ by $L_{c}=$ $q_{*}^{-1}\left(n_{*}(L)\right)$. We note that the image of $P\left(T_{p_{-}} \Sigma\right)$ (with the orientation induced from $\tilde{\Sigma}$ ) induces naturally an element in $H_{1}\left(\Sigma_{c}, \mathbb{Z}\right)$ and as such it is contained in $L_{c}$.

Remark 2.3. If we have two glueing maps $c_{i}: P\left(T_{p_{-}} \Sigma\right) \rightarrow P\left(T_{p_{+}} \Sigma\right), i=1,2$, we note that there is a diffeomorphism $f$ of $\Sigma$ inducing the identity on $\left(p_{-}, v_{-}\right) \sqcup\left(p_{+}, v_{+}\right) \sqcup(P, V)$ which is isotopic to the identity among such maps, such that $\left(d f_{p_{+}}\right)^{-1} c_{2} d f_{p_{-}}=c_{1}$. In particular $f$ induces a diffeomorphism 
$f: \Sigma_{c_{1}} \rightarrow \Sigma_{c_{2}}$ compatible with $f: \Sigma \rightarrow \Sigma$, which maps $L_{c_{1}}$ to $L_{c_{2}}$. Any two such diffeomorphisms of $\Sigma$ induces isotopic diffeomorphisms from $\Sigma_{1}$ to $\Sigma_{2}$.

Definition 2.8. Let $\boldsymbol{\Sigma}=\left(\Sigma,\left\{p_{-}, p_{+}\right\} \sqcup P,\left\{v_{-}, v_{+}\right\} \sqcup V, L\right)$ be a marked surface. Let

$$
c: P\left(T_{p_{-}} \Sigma\right) \rightarrow P\left(T_{p_{+}} \Sigma\right)
$$

be a glueing map and $\Sigma_{c}$ the glueing of $\Sigma$ at the ordered pair $\left(\left(p_{-}, v_{-}\right),\left(p_{+}, v_{+}\right)\right)$with respect to $c$. Let $L_{c} \subset H_{1}\left(\Sigma_{c}, \mathbb{Z}\right)$ be the Lagrangian subspace constructed above from $L$. Then the marked surface $\boldsymbol{\Sigma}_{c}=\left(\Sigma_{c}, P, V, L_{c}\right)$ is defined to be the glueing of $\boldsymbol{\Sigma}$ at the ordered pair $\left(\left(p_{-}, v_{-}\right),\left(p_{+}, v_{+}\right)\right)$ with respect to $c$.

We observe that glueing also extends to morphisms of marked surfaces which preserves the ordered pair $\left(\left(p_{-}, v_{-}\right),\left(p_{+}, v_{+}\right)\right)$, by using glueing maps which are compatible with the morphism in question.

We can now give the axioms for a 2 dimensional modular functor.

Definition 2.9. A label set $\Lambda$ is a finite set furnished with an involution $\lambda \mapsto \hat{\lambda}$ and a trivial element 1 such that $\hat{1}=1$.

Definition 2.10. Let $\Lambda$ be a label set. The category of $\Lambda$-labeled marked surfaces consists of marked surfaces with an element of $\Lambda$ assigned to each of the marked point and morphisms of labeled marked surfaces are required to preserve the labelings. An assignment of elements of $\Lambda$ to the marked points of $\boldsymbol{\Sigma}$ is called a labeling of $\boldsymbol{\Sigma}$ and we denote the labeled marked surface by $(\boldsymbol{\Sigma}, \lambda)$, where $\lambda$ is the labeling.

We define a labeled pointed surface similarly.

Remark 2.4. The operation of disjoint union clearly extends to labeled marked surfaces. When we extend the operation of orientation reversal to labeled marked surfaces, we also apply the involution $\hat{\imath}$ to all the labels.

Definition 2.11. A modular functor based on the label set $\Lambda$ is a functor $V$ from the category of labeled marked surfaces to the category of finite dimensional complex vector spaces satisfying the axioms MF1 to MF5 below.

MF1. Disjoint union axiom: The operation of disjoint union of labeled marked surfaces is taken to the operation of tensor product, i.e. for any pair of labeled marked surfaces there is an isomorphism

$$
\left.V\left(\left(\boldsymbol{\Sigma}_{1}, \lambda_{1}\right) \sqcup\left(\boldsymbol{\Sigma}_{2}, \lambda_{2}\right)\right)\right) \cong V\left(\boldsymbol{\Sigma}_{1}, \lambda_{1}\right) \otimes V\left(\boldsymbol{\Sigma}_{2}, \lambda_{2}\right) .
$$

The identification is associative. 
MF2. Glueing axiom: Let $\boldsymbol{\Sigma}$ and $\boldsymbol{\Sigma}_{c}$ be marked surfaces such that $\boldsymbol{\Sigma}_{c}$ is obtained from $\boldsymbol{\Sigma}$ by glueing at an ordered pair of points and projective tangent vectors with respect to a glueing map $c$. Then there is an isomorphism

$$
V\left(\boldsymbol{\Sigma}_{c}, \lambda\right) \cong \bigoplus_{\mu \in \Lambda} V(\boldsymbol{\Sigma}, \mu, \hat{\mu}, \lambda)
$$

which is associative, compatible with glueing of morphisms, disjoint unions and it is independent of the choice of the glueing map in the obvious way (see remark 2.3).

MF3. Empty surface axiom: Let $\emptyset$ denote the empty labeled marked surface. Then

$$
\operatorname{dim} V(\emptyset)=1
$$

MF4. Once punctured sphere axiom: Let $\boldsymbol{\Sigma}=\left(S^{2},\{p\},\{v\}, 0\right)$ be a marked sphere with one marked point. Then

$$
\operatorname{dim} V(\boldsymbol{\Sigma}, \lambda)= \begin{cases}1, & \lambda=1 \\ 0, & \lambda \neq 1\end{cases}
$$

MF5. Twice punctured sphere axiom: Let $\boldsymbol{\Sigma}=\left(S^{2},\left\{p_{1}, p_{2}\right\},\left\{v_{1}, v_{2}\right\},\{0\}\right)$ be a marked sphere with two marked points. Then

$$
\operatorname{dim} V(\boldsymbol{\Sigma},(\lambda, \mu))= \begin{cases}1, & \lambda=\hat{\mu} \\ 0, & \lambda \neq \hat{\mu} .\end{cases}
$$

In addition to the above axioms one may has extra properties, namely

$M F-D$. Orientation reversal axiom: The operation of orientation reversal of labeled marked surfaces is taken to the operation of taking the dual vector space, i.e for any labeled marked surface $(\boldsymbol{\Sigma}, \lambda)$ there is a pairing

$$
\langle\cdot, \cdot\rangle: V(\boldsymbol{\Sigma}, \lambda) \otimes V(-\boldsymbol{\Sigma}, \hat{\lambda}) \rightarrow \mathbb{C}
$$

compatible with disjoint unions, glueings and orientation reversals (in the sense that the induced isomorphisms $V(\boldsymbol{\Sigma}, \lambda) \cong V(-\boldsymbol{\Sigma}, \hat{\lambda})^{*}$ and $V(-\boldsymbol{\Sigma}, \hat{\lambda}) \cong V(\boldsymbol{\Sigma}, \lambda)^{*}$ are adjoints).

and

$M F-U$. Unitarity axiom Every vector space $V(\boldsymbol{\Sigma}, \lambda)$ is furnished with a hermitian inner product

$$
(\cdot, \cdot): V(\boldsymbol{\Sigma}, \lambda) \otimes \overline{V(\boldsymbol{\Sigma}, \lambda)} \rightarrow \mathbb{C}
$$

so that morphisms induces unitary transformation. The hermitian structure must be compatible with disjoint union and glueing. If we have the orientation reversal property, then compatibility 
with the unitary structure means that we have a commutative diagrams

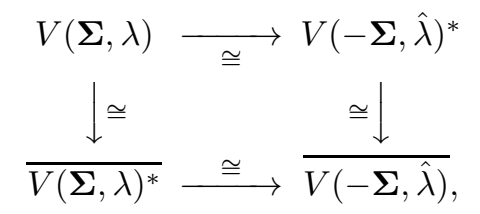

where the vertical identifications come from the hermitian structure and the horizontal from the duality.

The rest of the paper is concerned with the detailed geometric construction of modular functors using conformal field theory. However, we shall assume the reader is familiar with [19] and [2] and freely use the notations of these two papers in this paper.

\section{Teichmüller space and families of pointed Riemann Surfaces With formal NEIGHBOURHOODS}

Let us first review some basic Teichmüller theory. Let $\Sigma$ be a closed oriented smooth surface and let $P$ be finite set of points on $\Sigma$.

Definition 3.1. A marked Riemann surface $\mathbf{C}$ is a Riemann surface $C$ with a finite set of marked points $Q$ and non-zero tangent vectors $W \in T_{Q} C=\bigsqcup_{q \in Q} T_{q} C$.

Definition 3.2. A morphism between marked Riemann surface is a biholomorphism of the underlying Riemann surface which induces a bijection between the two sets of marked points and tangent vectors at the marked points.

The notions of stable and saturated is defined just like for pointed surfaces.

Definition 3.3. A complex structure on $(\Sigma, P)$ is a marked Riemann surface $\mathbf{C}=(C, Q, W)$ together with an orientation preserving diffeomorphism $\phi: \Sigma \rightarrow C$ mapping the points $P$ onto the points $Q$. Two such complex structures $\phi_{j}:(\Sigma, P) \rightarrow \mathbf{C}_{j}=\left(C_{j}, Q_{j}, W_{j}\right)$ are equivalent if there exists a morphism of marked Riemann surfaces

$$
\Phi: \mathbf{C}_{1} \rightarrow \mathbf{C}_{2}
$$

such that $\phi_{2}^{-1} \Phi \phi_{1}:(\Sigma, P) \rightarrow(\Sigma, P)$ is isotopic to the identity through maps inducing the identity on the first order neighbourhood of $P$.

We shall often in our notation suppress the diffeomorphism, when we denote a complex structure on a surface.

Definition 3.4. The Teichmüller space $\mathcal{T}_{(\Sigma, P)}$ of the pointed surface $(\Sigma, P)$ is by definition the set of equivalence classes of complex structures on $(\Sigma, P)$.

We note there is a natural projection map from $\mathcal{T}_{(\Sigma, P)}$ to $T_{P} \Sigma=\sqcup_{p \in P} T_{p} \Sigma$, which we call $\pi_{P}$. 
Theorem 3.1 (Bers). There is a natural structure of a finite dimensional complex analytic manifold on Teichmüller space $\mathcal{T}_{(\Sigma, P)}$. Associated to any morphism of pointed surfaces $f:\left(\Sigma_{1}, P_{1}\right) \rightarrow\left(\Sigma_{2}, P_{2}\right)$ there is a biholomorphism $f^{*}: \mathcal{T}_{\left(\Sigma_{1}, P_{1}\right)} \rightarrow \mathcal{T}_{\left(\Sigma_{2}, P_{2}\right)}$ which is induced by mapping a complex structure $\mathbf{C}=(C, Q, W), \phi: \Sigma_{1} \rightarrow C$ to $\phi \circ f^{-1}: \Sigma_{2} \rightarrow C$. Moreover, compositions of morphisms go to compositions of induced biholomorphisms.

There is an action of $\mathbb{R}_{+}^{\mathrm{P}}$ on $\mathcal{T}_{(\Sigma, P)}$ given by scaling the tangent vectors. This action is free and the quotient $\mathcal{T}^{(\mathrm{r})}{ }_{(\Sigma, P)}=\mathcal{T}_{(\Sigma, P)} / \mathbb{R}_{+}^{\mathrm{P}}$ is a smooth manifold, which we call the reduced Teichmüller space of the pointed surface $(\Sigma, P)$. Moreover the projection map $\pi_{P}$ descend to a smooth projection map from $\mathcal{T}^{(\mathrm{r})}{ }_{(\Sigma, P)}$ to $\sqcup_{p \in P} P\left(T_{p} \Sigma\right)$, which we denote $\pi_{P}^{(r)}$. We denote the fiber of this map over $V \in \sqcup_{p \in P} P\left(T_{p} \Sigma\right)$ by $\mathcal{T}_{(\Sigma, P, V)}$. Teichmüller space of a marked surface $\boldsymbol{\Sigma}=(\Sigma, P, V, L)$ is by definition $\mathcal{T}_{\boldsymbol{\Sigma}}=\mathcal{T}_{(\Sigma, P, V)}$, which we call the Teichmüller space of the marked surface. Morphisms of marked surfaces induce diffeomorphism of the corresponding Teichmüller spaces of marked surfaces, which of course also behaves well under composition. We observe that the self-morphism (Id, $s$ ) of a marked surface acts trivially on the associated Teichmüller space for all integers $s$. General Teichmüller theory implies that

Theorem 3.2. The Teichmüller space $\mathcal{T}_{\mathbf{\Sigma}}$ of any marked surface $\boldsymbol{\Sigma}$ is contractible.

Now let us recall the definition of a formal neighbourhood of a point on a Riemann surface.

Definition 3.5. Let $C$ be a Riemann surface and $q$ a point on $C$. Let $\mathcal{O}_{C, q}$ be the stalk of $\mathcal{O}_{C}$ at $q$ and let $\mathfrak{m}_{q}$ the maximal ideal in $\mathcal{O}_{C, q}$. We note that $\mathfrak{m}_{q}^{n}, n=0,1,2, \ldots$, gives a filtration of $\mathcal{O}_{C, q}$. A formal $n$ 'th-order neighbourhood at $q$ is a filtration preserving isomorphism

$$
\mathcal{O}_{C, q} / \mathfrak{m}_{q}^{n+1} \cong \mathbb{C}[[\xi]] /\left(\xi^{n+1}\right) .
$$

Let $\hat{\mathcal{O}}_{C, q}=\lim _{n \rightarrow \infty} \mathcal{O}_{C, q} / \mathfrak{m}_{q}^{n}$ be the completion of $\mathcal{O}_{C, q}$ with respect to the filtration. A formal neighbourhood (or formal coordinate) at $q$ is a filtration preserving isomorphism

$$
\eta: \hat{\mathcal{O}}_{C, q} \cong \mathbb{C}[[\xi]]
$$

We note that we have a canonical isomorphism

$$
\begin{aligned}
\mathcal{O}_{C, q} / \mathfrak{m}_{q}^{2} & \simeq \mathbb{C} \oplus T_{q}^{*} C, \\
f & \mapsto\left(f(q), d f_{q}\right) .
\end{aligned}
$$

Hence a formal 1'st order neighbourhood induces and is determined by an isomorphism of $T_{q}^{*} C$ with $\mathbb{C}$. Hence a formal 1'st order neighbourhood determines and is determined by a non-zero vector in $T_{q}^{*} C$, specified by the property that it maps to $1 \in \mathbb{C}$ or equivalently a vector in $T_{q} C$ pairing to unity with this vector. 
Definition 3.6. A pointed Riemann surface with formal neighbourhoods

$$
\mathfrak{X}=\left(C ; q_{1}, \ldots, q_{N} ; \eta_{1}, \ldots, \eta_{N}\right)
$$

is the following data: A Riemann surface $C$, an ordered $N$-tuple of $N$ distinct points $\left(q_{1}, \ldots, q_{N}\right)$ on $C$ together with formal neighbourhoods

$$
\eta_{j}: \hat{\mathcal{O}}_{C, q_{j}} \cong \mathbb{C}\left[\left[\xi_{j}\right]\right]
$$

for $j=1, \ldots, N$.

We remark that a pointed Riemann surface with formal neighbourhoods is an " $N$-pointed smooth curve with formal neighbourhoods" in the sense of Definition 1.1.3. of [19].

Definition 3.7. For a pointed Riemann surface with formal neighbourhoods $\mathfrak{X}$, we denote by $c(\mathfrak{X})$ the underlying marked Riemann surface. For a labeled pointed Riemann surface with formal neighbourhoods $(\mathfrak{X}, \vec{\lambda})$, we denote by $c(\mathfrak{X}, \vec{\lambda})=(c(\mathfrak{X}), \lambda)$ the underlying labeled marked Riemann surface. Here $\lambda$ denotes the labeling of the marked points of $c(\mathfrak{X})$ induced by $\vec{\lambda}$.

Definition 3.8. A family of pointed Riemann Surfaces with formal neighbourhoods

$$
\mathfrak{F}=(\pi: \mathcal{C} \rightarrow \mathcal{B} ; \vec{s} ; \vec{\eta})
$$

is the following date:

- Connected complex manifolds $\mathcal{C}$ and $\mathcal{B}$, such that $\operatorname{dim}_{\mathbb{C}} C=\operatorname{dim}_{\mathbb{C}} \mathcal{B}+1$.

- A holomorphic submersion $\pi: \mathcal{C} \rightarrow \mathcal{B}$.

- Holomorphic sections $s_{j}, j=1, \ldots, N$ of $\pi$.

- Filtered $\mathcal{O}_{\mathcal{B}}$-algebra isomorphisms

$$
\eta_{j}: \widehat{\mathcal{O}}_{/ s_{j}}=\lim _{n \rightarrow \infty} \mathcal{O}_{Y} / I_{j}^{n} \simeq \mathcal{O}_{\mathcal{B}}[[\xi]],
$$

where $I_{j}$ is the defining ideal of $s_{j}(\mathcal{B})$ in $\mathcal{C}, j=1, \ldots, N$.

Note that a family of pointed Riemann Surfaces with formal neighbourhoods is a "Family of $N$-pointed smooth curves with formal neighbourhoods" as in Definition 1.2.1 in [19]. See also the Appendix at the end of this paper.

Let $\Sigma$ be a closed oriented smooth surface and let $P$ be finite set of $N$ marked points on $\Sigma$, i.e. $(\Sigma, P)$ is a pointed surface.

For a connected smooth complex manifold $\mathcal{B}$ let $Y=\Sigma \times \mathcal{B}$.

Let $\mathfrak{F}=(\pi: \mathcal{C} \rightarrow \mathcal{B} ; \vec{s} ; \vec{\eta})$ be a family of pointed Riemann surfaces with formal neighbourhoods and assume we have a smooth fiber preserving diffeomorphism $\Phi_{\mathfrak{F}}$ from $Y$ to $\mathcal{C}$ taking the marked points to the sections $\vec{s}$ and inducing the identity on $\mathcal{B}$. This data induces a unique holomorphic map $\Psi_{\mathfrak{F}}$ from $\mathcal{B}$ to the Teichmüller space $\mathcal{T}_{(\Sigma, P)}$ of the surface $(\Sigma, P)$ by the universal property of Teichmüller space. 
Definition 3.9. The pair $\left(\mathfrak{F}, \Phi_{\mathfrak{F}}\right)$ is called a family of pointed Riemann surfaces with formal neighbourhoods on $(\Sigma, P)$. If $P^{\prime} \subset P$ is a strict subset, we say that $\left(\mathfrak{F}, \Phi_{\mathfrak{F}}\right)$ is called a family of pointed Riemann surfaces with formal neighbourhoods over $\left(\Sigma, P^{\prime}\right)$.

Often we will suppress $\Phi_{\mathfrak{F}}$ in our notation and just write $\mathfrak{F}$ is a family of pointed Riemann surfaces with formal neighbourhoods on $(\Sigma, P)$.

Definition 3.10. If a family $\mathfrak{F}=(\pi: \mathcal{C} \rightarrow \mathcal{B} ; \vec{s} ; \vec{\eta})$ of pointed Riemann surfaces with formal neighbourhoods on $(\Sigma, P)$, as above, has the properties, that the base $\mathcal{B}$ is biholomorphic to an open ball and that the induced map $\Psi_{\mathfrak{F}}$ is a biholomorphism onto an open subset of Teichmüller space $\mathcal{T}_{(\Sigma, P)}$ then the family is said to be good.

Note that if a family of pointed Riemann surfaces with formal neighbourhoods on $(\Sigma, P)$ is versal around some point $b \in B$, in the sense of Definition 1.2.2 in [19], then there is a open ball around $b$ in $B$, such that the restriction of the family to this neighbourhood is good.

Proposition 3.1. For a stable and saturated pointed surface $(\Sigma, P)$ the Teichmüller space $\mathcal{T}_{(\Sigma, P)}$ can be covered by images of such good families.

This follows from Theorem 1.2.9 in [19].

Suppose now that we have two stable and saturated families $\mathfrak{F}_{i}, i=1,2$ with the property that they have the same image $\Psi_{\mathfrak{F}_{1}}\left(\mathcal{B}_{1}\right)=\Psi_{\mathfrak{F}_{2}}\left(\mathcal{B}_{2}\right)$ in Teichmüller space $\mathcal{T}_{(\Sigma, P)}$ and that $\mathfrak{F}_{2}$ is a good family.

Proposition 3.2. For such a pair of families there exists a unique fiber preserving biholomorphism $\Phi: \mathcal{C}_{1} \rightarrow \mathcal{C}_{2}$ covering $\Psi_{\mathfrak{F}_{2}}^{-1} \Psi_{\mathfrak{F}_{1}}$ such that $\Phi_{\mathfrak{F}_{2}}^{-1} \Phi \Phi_{\mathfrak{F}_{1}}:(Y, P) \rightarrow(Y, P)$ is isotopic to $\Psi_{\mathfrak{F}_{2}}^{-1} \Psi_{\mathfrak{F}_{1}} \times \operatorname{Id}$ through such fiber preserving maps inducing the identity on the first order neighbourhood of $P$.

This follows from uniqueness of the $\Phi$ in Definition 3.3

We note that there is some permutation $S$ of $\{1, \ldots, N\}$ such that $\left(S \Phi^{*}\left(\vec{\eta}_{2}\right)\right)^{(1)}=\left(\vec{\eta}_{1}\right)^{(1)}$, i.e. $S \Phi^{*}\left(\vec{\eta}_{2}\right)$ induce the same first order formal neighbourhoods as $\vec{\eta}_{1}$ does.

Suppose now $f$ is an orientation preserving diffeomorphism from $\left(\Sigma_{1}, P_{1}\right)$ to $\left(\Sigma_{2}, P_{2}\right)$. Let $\mathfrak{F}_{1}$ be a family of pointed Riemann surfaces with formal neighbourhoods of $\left(\Sigma_{1}, P_{1}\right)$. By composing $\Phi_{\mathfrak{F}_{1}}$ with $f^{-1} \times$ Id we get a family of pointed Riemann surfaces with formal neighbourhoods of $\left(\Sigma_{2}, P_{2}\right)$. We note that $\Psi_{\mathfrak{F}_{2}}=f^{*} \circ \Psi_{\mathfrak{F}_{1}}$, where $f^{*}$ is the induced map between the Teichmüller spaces. This operation on families clearly behaves well under compositions of diffeomorphisms.

\section{The space of Vacua associated to a labeled marked Riemann Surface}

4.1. Affine Lie algebras and integrable highest weight modules. In this section we recall the basic facts about integrable highest weight representations of affine Lie algebras. For the details 
of integrable highest weight representations of affine Lie algebras we refer the reader to Kac's book $[\mathrm{Ka}]$.

Let $\mathfrak{g}$ be a simple Lie algebra over the complex numbers $\mathbb{C}$, which we fix throughout the paper. Let $\mathfrak{h}$ be its Cartan subalgebra. By $\Delta$ we denote the root system of $(\mathfrak{g}, \mathfrak{h})$. We have the root space decomposition

$$
\mathfrak{g}=\mathfrak{h} \oplus \sum_{\alpha \in \Delta} \mathfrak{g}_{\alpha} .
$$

Let $\mathfrak{h}_{\mathbf{R}}^{*}$ be the linear span of $\Delta$ over $\mathbf{R}$. Fix a choice of positive roots $\Delta_{+}$.

Let $(, \quad)$ be a constant multiple of the Cartan-Killing form of the simple Lie algebra $\mathfrak{g}$. For each element of $\lambda \in \mathfrak{h}$, there exists a unique element $H_{\lambda} \in \mathfrak{h}^{*}$ such that

$$
\lambda(H)=\left(H_{\lambda}, H\right)
$$

for all $H \in \mathfrak{h}$. For $\alpha \in \Delta, H_{\alpha}$ is called the root vector corresponding to the root $\alpha$.

On $\mathfrak{h}^{*}$ we introduce an inner product by

$$
(\lambda, \mu)=\left(H_{\lambda}, H_{\mu}\right) .
$$

Let us normalize the inner product $(, \quad$ ) by requiring that $\theta$, the highest (or longest) root, has length squared

$$
(\theta, \theta)=2 .
$$

Let $V_{\lambda}$ be the irreducible left $\mathfrak{g}$-module of highest weight $\lambda$. It is well-known that a finite dimensional irreducible left $\mathfrak{g}$-module is a highest weight module and two irreducible left $\mathfrak{g}$-modules are isomorphic if and only if they have the same highest weight. A weight $\lambda \in \mathfrak{h}_{\mathbf{R}}^{*}$ is called an integral weight, if

$$
2(\lambda, \alpha) /(\alpha, \alpha) \in \mathbf{Z}
$$

for any $\alpha \in \Delta$. A weight $\lambda \in \mathfrak{h}_{\mathbf{R}}^{*}$ is called a dominant weight, if

$$
w(\lambda) \leq \lambda
$$

for any element $w$ of the Weyl group $W$ of $\mathfrak{g}$. By $P_{+}$we denote the set of dominant integral weights of $\mathfrak{g}$. A weight $\lambda$ is the highest weight of an irreducible left $\mathfrak{g}$-module if and only if $\lambda \in P_{+}$.

Let $w$ be longest element of $W$. Then we define an involution $\dagger$ on $P_{+}$by

$$
\lambda^{\dagger}=-w(\lambda) \text {. }
$$

One has that the opposite of dual of the left-g-module $V_{\lambda}$ is isomorphic to left-g-module $V_{\lambda^{\dagger}}$, meaning there exists a non-degenerate $\mathfrak{g}$-invariant perfect pairing

$$
(, \quad): V_{\lambda} \otimes V_{\lambda^{\dagger}} \rightarrow \mathbb{C} .
$$

As mentioned in the introduction, we will need to fix $|0\rangle \in V_{0} \backslash\{0\}$ and

$$
\left|0_{\lambda, \lambda^{\dagger}}\right\rangle \in\left(V_{\lambda} \otimes V_{\lambda^{\dagger}}\right)^{\mathfrak{g}} \backslash\{0\},
$$


where we put $\left|0_{\lambda \lambda^{\dagger}}\right\rangle=|0\rangle \otimes|0\rangle$ for $\lambda=0$. Fixing such a vector is of course equivalent to fixing the above mentioned pairing.

By $\mathbf{C}[[\xi]]$ and $\mathbf{C}((\xi))$ we mean the ring of formal power series in $\xi$ and the field of formal Laurent power series in $\xi$, respectively.

Definition 4.1. The affine Lie algebra $\widehat{\mathfrak{g}}$ over $\mathbf{C}((\xi))$ associated with $\mathfrak{g}$ is defined to be

$$
\widehat{\mathfrak{g}}=\mathfrak{g} \otimes \mathbf{C}((\xi)) \oplus \mathbf{C} c
$$

where $c$ is an element of the center of $\widehat{\mathfrak{g}}$ and the Lie algebra structure is given by

$$
[X \otimes f(\xi), Y \otimes g(\xi)]=[X, Y] \otimes f(\xi) g(\xi)+c \cdot(X, Y) \underset{\xi=0}{\operatorname{Res}}(g(\xi) d f(\xi))
$$

for

$$
X, Y \in \mathfrak{g}, f(\xi), g(\xi) \in \mathbf{C}((\xi))
$$

Put

$$
\widehat{\mathfrak{g}}_{+}=\mathfrak{g} \otimes \mathbf{C}[[\xi]] \xi, \quad \widehat{\mathfrak{g}}_{-}=\mathfrak{g} \otimes \mathbf{C}\left[\xi^{-1}\right] \xi^{-1} .
$$

We regard $\widehat{\mathfrak{g}}_{+}$and $\widehat{\mathfrak{g}}_{-}$as Lie subalgebras of $\widehat{\mathfrak{g}}$. We have a decomposition

$$
\widehat{\mathfrak{g}}=\widehat{\mathfrak{g}}_{+} \oplus \mathfrak{g} \oplus \mathbf{C} c \oplus \widehat{\mathfrak{g}}_{-} .
$$

Let us fix a positive integer $\ell$ (called the level) and put

$$
P_{\ell}=\left\{\lambda \in P_{+} \mid 0 \leq(\theta, \lambda) \leq \ell\right\} .
$$

For all levels $\ell$ we observe that $\dagger$ takes $P_{\ell}$ to it self.

For each element $\lambda \in P_{\ell}$ we shall define the Verma module $\mathcal{M}_{\lambda}$ as follows. Put

$$
\widehat{\mathfrak{p}}_{+}:=\widehat{\mathfrak{g}}_{+} \oplus \mathfrak{g} \oplus \mathbf{C} \cdot c .
$$

Then $\widehat{\mathfrak{p}}_{+}$is a Lie subalgebra of $\widehat{\mathfrak{g}}$. Let $V_{\lambda}$ is the irreducible left $\mathfrak{g}$-module of highest weight $\lambda$. The action of $\widehat{\mathfrak{p}}_{+}$on $V_{\lambda}$ is defined as

$$
\begin{aligned}
& c v=\ell v \text { for all } v \in V_{\lambda} \\
& a v=0 \text { for all } a \in \widehat{\mathfrak{g}}_{+} \text {and } v \in V_{\lambda}
\end{aligned}
$$

Put

$$
\mathcal{M}_{\lambda}:=U(\widehat{\mathfrak{g}}) \otimes_{\widehat{\mathfrak{p}}_{+}} V_{\lambda}
$$

Then $\mathcal{M}_{\lambda}$ is a left $\widehat{\mathfrak{g}}$-module and is called a Verma module. The Verma module $\mathcal{M}_{\lambda}$ is not irreducible and contains the maximal proper submodule $\mathcal{J}_{\lambda}$. The quotient module $\mathcal{H}_{\lambda}:=\mathcal{M}_{\lambda} / \mathcal{J}_{\lambda}$ has the following properties. 
Theorem 4.1. For each $\lambda \in P_{\ell}$, the left $\widehat{\mathfrak{g}}$-module $\mathcal{H}_{\lambda}$ is the unique left $\widehat{\mathfrak{g}}$-module (called the integrable highest weight $\widehat{\mathfrak{g}}$-module) satisfying the following properties.

- $\left.V_{\lambda}=\left\{|v\rangle \in \mathcal{H}_{\lambda}\left|\widehat{\mathfrak{g}}_{+}\right| v\right\rangle=0\right\}$ is the irreducible left $\mathfrak{g}$-module with highest weight $\lambda$.

- The central element $c$ acts on $\mathcal{H}_{\lambda}$ as $\ell \cdot \mathrm{id}$.

- $\mathcal{H}_{\lambda}$ is generated by $V_{\lambda}$ over $\widehat{\mathfrak{g}}_{-}$with only one relation

$$
\left(X_{\theta} \otimes \xi^{-1}\right)^{\ell-(\theta, \lambda)+1}|\lambda\rangle=0
$$

where $X_{\theta} \in \mathfrak{g}$ is the element corresponding to the maximal root $\theta$ and $|\lambda\rangle \in V_{\lambda}$ is a highest weight vector.

The theorem says that the maximal proper submodule $\mathcal{J}_{\lambda}$ is given by

$$
\mathcal{J}_{\lambda}=U\left(\widehat{\mathfrak{p}}_{-}\right)\left|J_{\lambda}\right\rangle
$$

where we put

$$
\left|J_{\lambda}\right\rangle=\left(X_{\theta} \otimes \xi^{-1}\right)^{\ell-(\theta, \lambda)+1}|\lambda\rangle .
$$

For the details see (10.4.6) in [12.

Similarly we have the integrable lowest weight right $\widehat{\mathfrak{g}}$-module $\mathcal{H}_{\lambda}^{\dagger}$ which will be discussed below.

4.2. The Segal-Sugawara construction. We use the following notation

$$
\begin{aligned}
X(n) & =X \otimes \xi^{n}, \quad X \in \mathfrak{g} \\
X(z) & =\sum_{n \in \mathbf{Z}} X(n) z^{-n-1}
\end{aligned}
$$

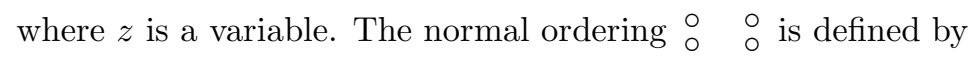

$$
\stackrel{\circ}{\circ}(n) Y(m) \stackrel{\circ}{\circ}= \begin{cases}X(n) Y(m), & n<m, \\ \frac{1}{2}(X(n) Y(m)+Y(m) X(n)) & n=m, \\ Y(m) X(n) & n>m .\end{cases}
$$

Note that, if $n>m$ and $X=Y$, we have

$$
{ }_{\circ}^{\circ} X(n) X(m) \stackrel{\circ}{\circ}=X(n) X(m)-n \delta_{n+m, 0}(X, X) \cdot c .
$$

Definition 4.2. The energy-momentum tensor $T(z)$ of level $\ell$ is defined by

$$
T(z)=\frac{1}{2\left(g^{*}+\ell\right)} \sum_{a=1}^{\operatorname{dim} \mathfrak{g}} \circ J^{a}(z) J^{a}(z) \stackrel{\circ}{\circ}
$$

where $\left\{J^{1}, J^{2}, \ldots, J^{\operatorname{dim} \mathfrak{g}}\right\}$ is an orthonormal basis of $\mathfrak{g}$ with respect to the Cartan-Killing form $(, \quad)$ and $g^{*}$ is the dual Coxeter number of $\mathfrak{g}$. 
Put

$$
L_{n}=\frac{1}{2\left(g^{*}+\ell\right)} \sum_{m \in \mathbf{Z}} \sum_{a=1}^{\operatorname{dim} \mathfrak{g}} \circ J^{a}(m) J^{a}(n-m)_{\circ}^{\circ} .
$$

Then we have the expansion

$$
T(z)=\sum_{n \in \mathbf{Z}} L_{n} z^{-n-2} .
$$

The operator $L_{n}$ is called the $n$ 'th Virasoro operator and it acts on $\mathcal{H}_{\lambda}$.

For $X \in \mathfrak{g}, f=f(z) \in \mathbb{C}((z))$ and $\underline{\ell}=\ell(z) \frac{d}{d z} \in \mathbb{C}((z)) \frac{d}{d z}$ we use the following notation.

$$
\begin{aligned}
X[f] & =\operatorname{Res}_{z=0}(X(z) f(z) d z) \\
T[\underline{\ell} & =\operatorname{Res}_{z=0}(T(z) \ell(z) d z) .
\end{aligned}
$$

In particular, we have that

$$
L_{0}=T\left[\xi \frac{d}{d \xi}\right]
$$

To define a filtration $\left\{F_{\bullet}\right\}$ on $\mathcal{H}_{\lambda}$, we first define the subspace $\mathcal{H}_{\lambda}(d)$ of $\mathcal{H}_{\lambda}$ for a non-negative integer $d$ by

$$
\left.\mathcal{H}_{\lambda}(d)=\left\{|v\rangle \in \mathcal{H}_{\lambda}\left|\quad L_{0}\right| v\right\rangle=\left(d+\Delta_{\lambda}\right)|v\rangle\right\}
$$

where

$$
\Delta_{\lambda}=\frac{(\lambda, \lambda)+2(\lambda, \rho)}{2\left(g^{*}+\ell\right)}, \quad \rho=\frac{1}{2} \sum_{\alpha \in \Delta_{+}} \alpha .
$$

The subspaces $\mathcal{H}_{\lambda}(d)$ are finite dimensional vector space and one has that

$$
\mathcal{H}_{\lambda}=\bigoplus_{d=0}^{\infty} \mathcal{H}_{\lambda}(d)
$$

Now we define the filtration $\left\{F_{p} \mathcal{H}_{\lambda}\right\}$ by

$$
F_{p} \mathcal{H}_{\lambda}=\sum_{d=0}^{p} \mathcal{H}_{\lambda}(d)
$$

Put

$$
\mathcal{H}_{\lambda}^{\dagger}(d)=\operatorname{Hom}_{\mathbf{C}}\left(\mathcal{H}_{\lambda}(d), \mathbb{C}\right)
$$

Then the dual space $\mathcal{H}_{\lambda}^{\dagger}$ of $\mathcal{H}_{\lambda}$ is defined to be

$$
\mathcal{H}_{\lambda}^{\dagger}=\operatorname{Hom}_{\mathbb{C}}\left(\mathcal{H}_{\lambda}, \mathbb{C}\right)=\prod_{d=0}^{\infty} \mathcal{H}_{\lambda}^{\dagger}(d) .
$$


By our definition $\mathcal{H}_{\lambda}^{\dagger}$ is a right $\widehat{\mathfrak{g}}$-module. A decreasing filtration $\left\{F^{p} \mathcal{H}_{\lambda}^{\dagger}\right\}$ is defined by

$$
F^{p} \mathcal{H}_{\lambda}^{\dagger}=\prod_{d \geq p} \mathcal{H}_{\lambda}^{\dagger}(d) .
$$

There is a unique canonical perfect bilinear pairing

$$
\langle\mid\rangle: \mathcal{H}_{\lambda}^{\dagger} \times \mathcal{H}_{\lambda} \longrightarrow \mathbb{C}
$$

given on $V_{\lambda}^{\dagger} \otimes V_{\lambda}$ by evaluation and which satisfies the following equality for each $a \in \widehat{\mathfrak{g}}$.

$$
\langle u \mid a v\rangle=\langle u a \mid v\rangle, \quad \text { for all }\langle u| \in \mathcal{H}_{\lambda}^{\dagger} \text { and }|v\rangle \in \mathcal{H}_{\lambda}
$$

Put

$$
V_{\lambda}^{\dagger}=\left\{\left\langle v\left|\in \mathcal{H}_{\lambda}^{\dagger}\right|\langle v| \widehat{\mathfrak{g}}_{-}=0\right\} .\right.
$$

It is easy to show that $V_{\lambda}^{\dagger}=\mathcal{H}_{\lambda}^{\dagger}(0)$ and $V_{\lambda}^{\dagger}$ is the irreducible right $\mathfrak{g}$-module with lowest weight $\lambda$. The integrable highest weight right $\widehat{\mathfrak{g}}$-module with lowest weight $\lambda$ is generated by $V_{\lambda}^{\dagger}$ over $\widehat{\mathfrak{g}}_{+}$with only one relation

$$
\langle\lambda|\left(X_{-\theta} \otimes \xi\right)^{\ell-(\theta, \lambda)+1}=0 .
$$

Now let us introduce the left $\mathfrak{g}$-module structure on $\mathcal{H}_{\lambda}^{\dagger}$ by

$$
X(n)\langle\Phi|:=-\langle\Phi| X(-n)
$$

It is easy to check that this indeed defines the left $\mathfrak{g}$-module structure on $\mathcal{H}_{\lambda}^{\dagger}$.

Now we give the relationship of the left $\mathfrak{g}$-module $\mathcal{H}_{\lambda}^{\dagger}$ and $\mathcal{H}_{\lambda^{\dagger}}$.

Lemma 4.1. There exists a unique canonical bilinear pairing

$$
(\mid): \mathcal{H}_{\lambda} \times \mathcal{H}_{\lambda^{\dagger}} \rightarrow \mathbf{C}
$$

such that we have

$$
(X(n) u \mid v)+(u \mid X(-n) v)=0
$$

for any $X \in \mathfrak{g}, n \in \mathbf{Z},|u\rangle \in \mathcal{H}_{\lambda},|v\rangle \in \mathcal{H}_{\lambda^{\dagger}}$, the pairing is zero on $\mathcal{H}_{\lambda}(d) \times \mathcal{H}_{\lambda^{\dagger}}\left(d^{\prime}\right)$, if $d \neq d^{\prime}$ and it evaluates to 1 on $\left|0_{\lambda, \lambda^{\dagger}}\right\rangle$.

Corollary 4.1. This pairing induces a canonical left $\mathfrak{g}$-module isomorphism

$$
\mathcal{H}_{\lambda}^{\dagger} \simeq \widehat{\mathcal{H}}_{\lambda^{\dagger}}
$$

where $\widehat{\mathcal{H}}_{\lambda^{\dagger}}$ is the completion of $\mathcal{H}_{\lambda^{\dagger}}$ with respect to the filtration $\left\{F_{p}\right\}$. 


\subsection{The space of vacua.}

Definition 4.3. The Lie algebra $\widehat{\mathfrak{g}}_{N}$ is defined as

$$
\widehat{\mathfrak{g}}_{N}=\bigoplus_{j=1}^{N} \mathfrak{g} \otimes_{\mathbb{C}} \mathbb{C}\left(\left(\xi_{j}\right)\right) \oplus \mathbb{C} c
$$

with the following commutation relations.

$$
\left[\left(X_{j} \otimes f_{j}\right),\left(Y_{j} \otimes g_{j}\right)\right]=\left(\left[X_{j}, Y_{j}\right] \otimes f_{j} g_{j}\right)+c \sum_{j=1}^{N}\left(X_{j}, Y_{j}\right) \underset{\xi_{j}=0}{\operatorname{Res}}\left(g_{j} d f_{j}\right)
$$

where $\left(a_{j}\right)$ means $\left(a_{1}, a_{2}, \ldots, a_{N}\right)$ and $c$ belongs to the center of $\widehat{\mathfrak{g}}_{N}$.

Let $\mathfrak{X}=\left(C ; q_{1}, q_{2}, \ldots, q_{N} ; \eta_{1}, \eta_{2}, \ldots, \eta_{N}\right)$ be a pointed saturated Riemann Surface with formal neighbourhoods and define

$$
\widehat{\mathfrak{g}}(\mathfrak{X})=\mathfrak{g} \otimes_{\mathbb{C}} H^{0}\left(C, \mathcal{O}_{C}\left(* \sum_{j=1}^{N} q_{j}\right)\right) .
$$

We have the natural embedding

$$
t=\oplus t_{i}: H^{0}\left(C, \mathcal{O}_{C}\left(* \sum_{j=1}^{N} q_{j}\right)\right) \hookrightarrow \bigoplus_{j=1}^{N} \mathbb{C}\left(\left(\xi_{j}\right)\right)
$$

given by Laurent expansion using the formal neighbourhoods. In the following we often regard $H^{0}\left(C, \mathcal{O}_{C}\left(* \sum_{j=1}^{N} q_{j}\right)\right)$ as a subspace of $\bigoplus_{j=1}^{N} \mathbb{C}\left(\left(\xi_{j}\right)\right)$. One has by lemma 1.1 .15 in [19], that $\widehat{\mathfrak{g}}(\mathfrak{X})$ is a Lie subalgebra of $\widehat{\mathfrak{g}}_{N}$.

Let us fix a non-negative integer $\ell$. For each $\vec{\lambda}=\left(\lambda_{1}, \ldots, \lambda_{N}\right) \in\left(P_{\ell}\right)^{N}$, the left $\widehat{\mathfrak{g}}_{N}$-module $\mathcal{H}_{\vec{\lambda}}$ and a right $\widehat{\mathfrak{g}}_{N}$-module $\mathcal{H}_{\vec{\lambda}}^{\dagger}$ are defined by

$$
\begin{aligned}
\mathcal{H}_{\vec{\lambda}} & =\mathcal{H}_{\lambda_{1}} \otimes_{\mathbb{C}} \cdots \otimes_{\mathbb{C}} \mathcal{H}_{\lambda_{N}} \\
\mathcal{H}_{\vec{\lambda}}^{\dagger} & =\mathcal{H}_{\lambda_{1}}^{\dagger} \widehat{\otimes}_{\mathbb{C}} \cdots \widehat{\otimes}_{\mathbb{C}} \mathcal{H}_{\lambda_{N}}^{\dagger} .
\end{aligned}
$$

The hats over the tensor product means that the algebraic tensor product has been completed with respect to the induced filtration.

For each element $X_{j} \in \mathfrak{g}, f\left(\xi_{j}\right) \in \mathbb{C}\left(\left(\xi_{j}\right)\right)$, the action $\rho_{j}$ of $X_{j}\left[f_{j}\right]$ on $\mathcal{H}_{\vec{\lambda}}$ is given by

$$
\rho_{j}\left(X_{j}\left[f_{j}\right]\right)\left|v_{1} \otimes \cdots \otimes v_{N}\right\rangle=\left|v_{1} \otimes \cdots \otimes v_{j-1} \otimes\left(X_{j}\left[f_{j}\right]\right) v_{j} \otimes v_{j+1} \otimes \cdots v_{N}\right\rangle
$$

where $\left|v_{1} \otimes \cdots \otimes v_{N}\right\rangle$ means $\left|v_{1}\right\rangle \otimes \cdots \otimes\left|v_{N}\right\rangle,\left|v_{j}\right\rangle \in \mathcal{H}_{\lambda_{j}}$. The left $\widehat{\mathfrak{g}}_{N}$-action is given by

$$
\left(X_{1} \otimes f_{1}, \ldots, X_{N} \otimes f_{N}\right)\left|v_{1} \otimes \cdots v_{N}\right\rangle=\sum_{j=1}^{N} \rho_{j}\left(X_{j}\left[f_{j}\right]\right)\left|v_{1} \otimes \cdots v_{N}\right\rangle .
$$


Similarly, the right $\widehat{\mathfrak{g}}_{N}$-action on $\mathcal{H}_{\vec{\lambda}}^{\dagger}$ is defined by

$$
\left\langle u_{1} \otimes \cdots u_{N}\right|\left(X_{1} \otimes f_{1}, \ldots, X_{N} \otimes f_{N}\right)=\sum_{j=1}^{N}\left\langle u_{1} \otimes \cdots u_{N}\right| \rho_{j}\left(X_{j}\left[f_{j}\right]\right) .
$$

As a Lie subalgebra, $\widehat{\mathfrak{g}}(\mathfrak{X})$ operates on $\mathcal{H}_{\vec{\lambda}}$ and $\mathcal{H}_{\vec{\lambda}}^{\dagger}$ as

$$
(X \otimes f)\left|v_{1} \otimes \cdots \otimes v_{N}\right\rangle=\sum_{j=1}^{N} \rho_{j}\left(X \otimes t_{j}(f)\right)\left|v_{1} \otimes \cdots v_{N}\right\rangle
$$

and as

$$
\left\langle u_{1} \otimes \cdots \otimes u_{N}\right|(X \otimes f)=\sum_{j=1}^{N}\left\langle u_{1} \otimes \cdots \otimes u_{N}\right| \rho_{j}\left(X \otimes t_{j}(f)\right) .
$$

The pairing $\langle\mid\rangle$ introduced in 18 induces a perfect bilinear pairing

$$
\langle\mid\rangle: \mathcal{H}_{\vec{\lambda}}^{\dagger} \times \mathcal{H}_{\vec{\lambda}} \rightarrow \mathbb{C}
$$

given by

$$
\left(\left\langle u_{1} \otimes \ldots \otimes u_{N}|,| v_{1} \otimes \ldots \otimes u_{N}\right\rangle\right) \rightarrow\left\langle u_{1} \mid v_{1}\right\rangle\left\langle u_{2} \mid v_{2}\right\rangle \cdots\left\langle u_{N} \mid v_{N}\right\rangle
$$

which is $\widehat{\mathfrak{g}}_{N}$-invariant:

$$
\left\langle\Psi\left(X_{j} \otimes f_{j}\right) \mid \Phi\right\rangle=\left\langle\Psi \mid\left(X_{j} \otimes f_{j}\right) \Phi\right\rangle .
$$

Now we are ready to define the space of vacua attached to $\mathfrak{X}$.

Definition 4.4. Assume that $\mathfrak{X}$ is saturated. Put

$$
\mathcal{V}_{\vec{\lambda}}(\mathfrak{X})=\mathcal{H}_{\vec{\lambda}} / \widehat{\mathfrak{g}}(\mathfrak{X}) \mathcal{H}_{\vec{\lambda}} .
$$

The vector space $\mathcal{V}_{\vec{\lambda}}(\mathfrak{X})$ is called the space of covacua attached to $\mathfrak{X}$. The space of vacua attached to $\mathfrak{X}$ is defined as

$$
\mathcal{V}_{\vec{\lambda}}^{\dagger}(\mathfrak{X})=\operatorname{Hom}_{\mathbb{C}}\left(\mathcal{V}_{\vec{\lambda}}(\mathfrak{X}), \mathbb{C}\right)
$$

One gets that

$$
\mathcal{V}_{\vec{\lambda}}^{\dagger}(\mathfrak{X})=\left\{\left\langle\Psi\left|\in \mathcal{H}_{\vec{\lambda}}^{\dagger}\right|\langle\Psi| \widehat{\mathfrak{g}}(\mathfrak{X})=0\right\} .\right.
$$

Moreover, the pairing (28) induces a perfect pairing

$$
\langle\mid\rangle: \mathcal{V}_{\vec{\lambda}}^{\dagger}(\mathfrak{X}) \times \mathcal{V}_{\vec{\lambda}}(\mathfrak{X}) \rightarrow \mathbb{C}
$$

The following theorem is proved in [19].

Theorem 4.2. The vector spaces $\mathcal{V}_{\vec{\lambda}}(\mathfrak{X})$ and $\mathcal{V}_{\vec{\lambda}}^{\dagger}(\mathfrak{X})$ are finite-dimensional. 
4.4. Propagation of vacua. For a pointed Riemann Surface with formal neighbourhoods $\mathfrak{X}=$ $\left(C ; q_{1}, \ldots, q_{N} ; \eta_{1}, \ldots, \eta_{N}\right)$ let $q_{N+1}$ be a point on $C \backslash\left\{q_{1}, \ldots, q_{N}\right\}$ and $\eta_{N+1}$ a formal neighbourhood of $C$ at $q_{N+1}$. Put

$$
\widetilde{\mathfrak{X}}=\left(C ; q_{1}, \ldots, q_{N}, q_{N+1} ; \eta_{1}, \ldots, \eta_{N}, \eta_{N+1}\right) .
$$

Since there is a canonical inclusion

$$
\begin{aligned}
\mathcal{H}_{\vec{\lambda}} & \longrightarrow \mathcal{H}_{\vec{\lambda}} \otimes \mathcal{H}_{0} \\
|v\rangle & \longrightarrow|v\rangle \otimes|0\rangle
\end{aligned}
$$

we have a canonical surjection

$$
\widehat{\iota}^{*}: \mathcal{H}_{\vec{\lambda}}^{\dagger} \widehat{\otimes} \mathcal{H}_{0}^{\dagger} \longrightarrow \mathcal{H}_{\vec{\lambda}}^{\dagger}
$$

Theorem 4.3. The canonical surjection $\widehat{\iota}^{*}$ induces a canonical Propagation of vacua isomorphism

$$
P_{\widetilde{\mathfrak{X}}, \mathfrak{X}}: \mathcal{V}_{\vec{\lambda}, 0}^{\dagger}(\widetilde{\mathfrak{X}}) \rightarrow \mathcal{V}_{\vec{\lambda}}^{\dagger}(\mathfrak{X})
$$

4.5. Change of formal neighbourhoods. We let $\mathcal{D}$ be the automorphism group Aut $\mathbb{C}((\xi))$ of the field $\mathbb{C}((\xi))$ of formal Laurent series as a $\mathbb{C}$-algebra. There is a natural isomorphism

$$
\begin{aligned}
\mathcal{D} & \simeq\left\{\sum_{n=0}^{\infty} a_{n} \xi^{n+1} \mid a_{0} \neq 0\right\} \\
h & \mapsto h(\xi)
\end{aligned}
$$

where the composition $h \circ g$ of $h, g \in \mathcal{D}$ corresponds to the formal power series $h(g(\xi))$.

Put

$$
\mathcal{D}^{p}=\left\{h \in \mathcal{D} \mid h(\xi)=\xi+a_{p} \xi^{p+1}+\cdots\right\}
$$

for a positive integer $p$. Then we have a filtration

$$
\mathcal{D}=\mathcal{D}^{0} \supset \mathcal{D}^{1} \supset \mathcal{D}^{2} \supset \ldots
$$

Also let

$$
\begin{aligned}
\underline{d} & =\mathbb{C}[[\xi]] \xi \frac{d}{d \xi} \\
\underline{d}^{p} & =\mathbb{C}[[\xi]] \xi^{p+1} \frac{d}{d \xi} \quad p=0,1,2, \ldots
\end{aligned}
$$

Then, we have a filtration

$$
\underline{d}=\underline{d}^{0} \supset \underline{d}^{1} \supset \underline{d}^{2} \supset \cdots
$$

For any $\underline{l} \in \underline{d}$ and $f(\xi) \in \mathbb{C}[[\xi]]$ define $\exp (\underline{l})(f(\xi))$ by

$$
\exp (\underline{l})(f(\xi))=\sum_{k=0}^{\infty} \frac{1}{k !}\left(\underline{l}^{k} f(\xi)\right) .
$$


Set

$$
\begin{aligned}
& \mathcal{D}_{+}^{0}=\left\{h \in \mathcal{D} \mid h(\xi)=a \xi+a_{1} \xi^{2}+\cdots, \quad a>0\right\} \\
& \underline{d}_{+}^{0}=\left\{l(\xi) \frac{d}{d \xi} \mid l(\xi)=\alpha \xi+\alpha_{1} \xi^{2}+\cdots, \quad \alpha \in \mathbb{R}\right\}
\end{aligned}
$$

Then, we have the following result.

Lemma 4.2. The exponential map

$$
\begin{aligned}
\exp : \underline{d} & \rightarrow \mathcal{D} \\
\underline{l} & \mapsto \exp (\underline{l})
\end{aligned}
$$

is surjective. Moreover, the exponential map induces an isomorphism

$$
\exp : \underline{d}_{+}^{0} \simeq \mathcal{D}_{+}^{0} .
$$

Since, for any integer $n$, we have

$$
\exp \left(2 \pi n \sqrt{-1} \xi \frac{d}{d \xi}\right)=i d
$$

the exponential mapping is not injective on $\underline{d}$.

For any element $\underline{l} \in \mathcal{D}_{+}^{0}$ we $\operatorname{define} \exp (T[\underline{l}])$ by

$$
\exp (T[\underline{l}])=\sum_{k=0}^{\infty} \frac{1}{k !} T\left[\underline{l}^{k} .\right.
$$

Then, $\exp (T[\underline{l}])$ operates on $\mathcal{H}_{\lambda}$ from the left and on $\mathcal{H}_{\lambda}^{\dagger}$ from the right.

By Lemma 4.2 for any automorphism $h \in \mathcal{D}_{+}^{0}$, there exist a unique $\underline{l} \in \underline{d}_{+}^{0}$ with $\exp (\underline{l})=h$. Now for $h \in \mathcal{D}_{+}^{0}$ define the operator $G[h]$ by

$$
G[h]=\exp (-T[\underline{l}])
$$

where $\exp (\underline{l})=h$. Then, $G[h]$ operates on $\mathcal{H}_{\lambda}$ from the left and on $\mathcal{H}_{\lambda}^{\dagger}$ from the right. Then the following important theorems hold.

Theorem 4.4 ([19, Theorem 3.2.4]). For any $h \in \mathcal{D}_{+}^{0}, f(\xi) d \xi \in \mathbf{C}((\xi)) d \xi, g(\xi) \in \mathbf{C}((\xi))$ and $\underline{l}=l(\xi) \frac{d}{d \xi} \in \mathbf{C}((\xi)) \frac{d}{d \xi}$, we have the following equalities as operators on $\mathcal{F}$ and $\mathcal{F}^{\dagger}$.

(1) $\quad G[h](\psi[f(\xi) d \xi]) G[h]^{-1}=\psi\left[h^{*}(f(\xi) d \xi)\right]=\psi\left[f(h(\xi)) h^{\prime}(\xi) d \xi\right]$

(2) $\quad G[h](\bar{\psi}[g(\xi)]) G[h]^{-1}=\bar{\psi}\left[h^{*}(g(\xi))\right]=\bar{\psi}[g(h(\xi))]$

(3) $\quad G\left[h_{1} \circ h_{2}\right]=G\left[h_{1}\right] G\left[h_{2}\right]$

(4) $G[h] T[\underline{l}] G[h]^{-1}=T[\operatorname{ad}(h)(\underline{l})]+\frac{1}{6} \underset{\xi=0}{\operatorname{Res}}(\{h(\xi) ; \xi\} l(\xi) d \xi)$.

where $\{f(\xi) ; \xi\}$ is the Schwarzian derivative. 
Theorem 4.5 ([19, Theorem 3.2.5]). For any $h_{j} \in \mathcal{D}_{+}^{0}, j=1,2, \ldots, N$ and a pointed Riemann surface with formal neighbourhoods

$$
\mathfrak{X}=\left(C ; q_{1}, q_{2}, \ldots, q_{N} ; \xi_{1}, \xi_{2}, \ldots, \xi_{N}\right)
$$

put

$$
\mathfrak{X}_{(h)}=\left(C ; q_{1}, q_{2}, \ldots, q_{N} ; h_{1}\left(\xi_{1}\right), h_{2}\left(\xi_{2}\right), \ldots, h_{N}\left(\xi_{N}\right)\right) .
$$

Then, the isomorphism $G\left[h_{1}\right] \widehat{\otimes} \cdots \widehat{\otimes} G\left[h_{N}\right]$

$$
\begin{aligned}
\mathcal{H}_{\vec{\lambda}}^{\dagger} & \rightarrow \mathcal{H}_{\vec{\lambda}}^{\dagger} \\
\left\langle\phi_{1} \widehat{\otimes} \cdots \widehat{\otimes} \phi_{N}\right| & \mapsto\left\langle\phi_{1} G\left[h_{1}\right] \widehat{\otimes} \cdots \widehat{\otimes} \phi_{N} G\left[h_{N}\right]\right|
\end{aligned}
$$

induces the canonical isomorphism

$$
G[\vec{h}]=G\left[h_{1}\right] \widehat{\otimes} \cdots \widehat{\otimes} G\left[h_{N}\right]: \mathcal{V}_{\vec{\lambda}}^{\dagger}(\mathfrak{X}) \rightarrow \mathcal{V}_{\vec{\lambda}}^{\dagger}\left(\mathfrak{X}_{(h)}\right)
$$

Let $\mathfrak{X}=(C ; \vec{Q} ; \vec{\eta})$ be a Riemann surface with formal neighbourhoods and let $q_{N+1}$ be a further point on the curve $C$ and $\eta_{N+1}$ a formal neighbourhood of $C$ at $q_{N+1}$. Put $\overrightarrow{\widetilde{Q}}=\left(q_{1}, \ldots, q_{N}, q_{N+1}\right)$ and $\overrightarrow{\widetilde{\eta}}=\left(\eta_{1}, \ldots, \eta_{N}, \eta_{N+1}\right)$. Let

$$
\widetilde{\mathfrak{X}}=(C ; \overrightarrow{\widetilde{Q}} ; \overrightarrow{\tilde{\eta}}) \text {. }
$$

We have the canonical isomorphism $P_{\widetilde{\mathfrak{X}}, \mathfrak{X}}$ from $\mathcal{V}_{\vec{\lambda}, 0}^{\dagger}(\widetilde{\mathfrak{X}})$ to $\mathcal{V}_{\vec{\lambda}}^{\dagger}(\mathfrak{X})$ as given in Theorem4.3, Suppose now $\vec{\xi}$ is another formal neighbourhood at $\vec{Q}$ and that $\xi_{N+1}$ is a formal neighbourhood at $q_{N+1}$. Let then $\overrightarrow{\widetilde{\xi}}=\left(\xi, \xi_{N+1}\right), \mathfrak{X}^{\prime}=(C ; \vec{Q} ; \vec{\xi})$ and $\widetilde{\mathfrak{X}}=(C ; \overrightarrow{\widetilde{Q}} ; \overrightarrow{\widetilde{\xi}})$. Let $\vec{h}$ be the formal coordinate change $\vec{\xi}=\vec{h}(\vec{\eta})$ and $\widetilde{\vec{h}}$ the formal coordinate change $\overrightarrow{\widetilde{\xi}}=\overrightarrow{\widetilde{h}}(\overrightarrow{\widetilde{\eta}})$. Assume now $c(\mathfrak{X}, \vec{\lambda})=c\left(\mathfrak{X}^{\prime}, \vec{\lambda}\right)$ and $c(\widetilde{\mathfrak{X}}, \vec{\lambda}, 0)=c\left(\widetilde{\mathfrak{X}^{\prime}}, \vec{\lambda}, 0\right)$. Then $h_{j} \in \mathcal{D}^{p_{j}}, p_{j} \geq 1$ and $\widetilde{h}_{j} \in \mathcal{D}^{\widetilde{p}_{j}}, \widetilde{p}_{j} \geq 1$. We then get the following diagram:

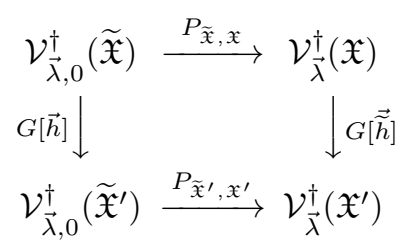

Proposition 4.1. The diagram (33) is commutative.

Proof. A simple explicit calculation shows that

$$
L_{k}|0\rangle=0
$$

if $k>0$ and

$$
L_{0}|0\rangle=\Delta_{0}|0\rangle \text {. }
$$


But by (15) $\Delta_{0}=0$. From this we immediately get that

$$
G\left[\tilde{h}_{N+1}\right]|0\rangle=|0\rangle,
$$

which by the very construction of the propagation of vacua isomorphism makes the above diagram commute.

4.6. The definition of the space of vacua associated to a labeled marked Riemann surface. Let $\mathbf{C}=(C, Q, W)$ be a pointed Riemann surface and let $\lambda$ be a labeling of $\mathbf{C}$. We shall now define the space of vacua attached to the pair $(\mathbf{C}, \lambda)$. We do this by providing canonical isomorphisms between the spaces of vacua associated to all correspondingly label pointed Riemann surfaces with formal neighbourhoods over $\mathbf{C}$. First we treat the case where $\mathbf{C}$ is saturated.

We notice that the definition of the space of vacua $\mathcal{V}_{\vec{\lambda}}^{\dagger}(\mathfrak{X})$ associated to a pointed Riemann surface with formal neighbourhoods $\mathfrak{X}=(C, \vec{Q}, \vec{\eta})$ depends on the ordering of the marked points $\vec{Q}=\left(q_{1}, \ldots, q_{N}\right)$. Let $S$ be a permutation of $\{1, \ldots, N\}$. We then define $\mathfrak{X}_{S}=(C, S(v Q), S(\vec{\eta}))$. The permutation $S$ acting from $\mathcal{H}_{\vec{\lambda}}$ to $\mathcal{H}_{S(\vec{\lambda})}$, induces an isomorphism

$$
S: \mathcal{V}_{\vec{\lambda}}^{\dagger}(\mathfrak{X}) \rightarrow \mathcal{V}_{S(\vec{\lambda})}^{\dagger}\left(\mathfrak{X}_{S}\right)
$$

Clearly, compositions of permutations go to compositions of isomorphisms.

Let $\mathfrak{X}^{\prime}=(C, \vec{Q}, \vec{\eta})$ and $\mathfrak{X}^{\prime}=\left(C, \vec{Q}^{\prime}, \vec{\eta}^{\prime}\right)$ be two pointed Riemann surface with formal neighbourhoods such that $c(\mathfrak{X})=\mathbf{C}=c\left(\mathfrak{X}^{\prime}\right)$. Let $S$ be such that $S(\vec{Q})=\vec{Q}^{\prime}$. Let $\vec{h}$ be the formal change of coordinates from $S(\vec{\eta})$ to $\vec{\eta}^{\prime}$. Then as discussed above we have the isomorphism

$$
G[\vec{h}] S: \mathcal{V}_{\vec{\lambda}}^{\dagger}(\mathfrak{X}) \rightarrow \mathcal{V}_{S(\vec{\lambda})}^{\dagger}\left(\mathfrak{X}^{\prime}\right)
$$

to identify the two spaces of vacua with.

Definition 4.5. Let $\mathbf{C}=(C, Q, W)$ be a saturated marked Riemann surface. Let $\lambda$ be a labeling of $\mathbf{C}$ using the set $P_{\ell}$. The space of vacua associated to the labeled marked curve $(\mathbf{C}, \lambda)$ is by definition

$$
\mathcal{V}_{\lambda}^{\dagger}(\mathbf{C})=\coprod_{c(\mathfrak{X}, \vec{\lambda})=(\mathbf{C}, \lambda)} \mathcal{V}_{\vec{\lambda}}^{\dagger}(\mathfrak{X}) / \sim,
$$

where the disjoint union is over all labeled curves with formal neighbourhoods with $(\mathbf{C}, \lambda)$ as the underlying labeled marked curve, $\vec{\lambda}$ is compatible with the labeling $\lambda$ and $\sim$ is the equivalence relation generated by the preferred isomorphisms (34).

That the relation $\sim$ is an equivalence relation follows from (1) and (2) in Theorem 4.4, Further it is clear that

Proposition 4.2. The natural quotient map from $\mathcal{V}_{\vec{\lambda}}^{\dagger}(\mathfrak{X})$ to $\mathcal{V}_{\lambda}^{\dagger}(\mathbf{C})$ is an isomorphism for all labeled Riemann surfaces with formal neighbourhoods $(\mathfrak{X}, \vec{\lambda})$ with $c(\mathfrak{X}, \vec{\lambda})=(\mathbf{C}, \lambda)$. 
Suppose $\left(\mathbf{C}_{i}, \lambda_{i}\right)$ are labeled marked Riemann surfaces and $\Phi:\left(\mathbf{C}_{1}, \lambda_{1}\right) \rightarrow\left(\mathbf{C}_{2}, \lambda_{2}\right)$ is a morphism of labeled marked Riemann Surfaces. Let $\left(\mathfrak{X}_{2}, \vec{\lambda}\right)$ be a labeled pointed Riemann Surface with formal neighbourhoods such that $c\left(\mathfrak{X}_{2}, \vec{\lambda}_{2}\right)=\left(\mathbf{C}, \lambda_{2}\right)$. Let $\Phi^{*} \mathfrak{X}_{2}=\mathfrak{X}_{1}$. Then $\Phi$ is a morphism of labeled marked Riemann surfaces with formal neighbourhoods. We obviously have that

Proposition 4.3. The identity map on $\mathcal{H}_{\vec{\lambda}}^{\dagger}$ induces a linear isomorphism from $\mathcal{V}_{\vec{\lambda}}^{\dagger}\left(\mathfrak{X}_{1}\right)$ to $\mathcal{V}_{\vec{\lambda}}^{\dagger}\left(\mathfrak{X}_{2}\right)$, which induces a well defined linear isomorphism $\mathcal{V}^{\dagger}(\Phi)$ from $\mathcal{V}_{\lambda_{1}}^{\dagger}\left(\mathbf{C}_{1}\right)$ to $\mathcal{V}_{\lambda_{2}}^{\dagger}\left(\mathbf{C}_{2}\right)$. Compositions of morphisms of labeled marked Riemann Surfaces go to compositions of the induced linear isomorphisms.

Let $(\mathbf{C}, \lambda)$ be a labeled marked Riemann surface which might not be saturated.

Consider all saturated labeled marked Riemann surfaces $\left(\mathbf{C}^{\prime}, \lambda^{\prime}\right)$ obtained from $(\mathbf{C}, \lambda)$ by adding points labeled with the trivial label $0 \in P_{\ell}$.

Definition 4.6. The space of vacua associated to the labeled marked Riemann surface $(\mathbf{C}, \lambda)$ is by definition

$$
\mathcal{V}_{\lambda}^{\dagger}(\mathbf{C})=\coprod_{\left(\mathbf{C}^{\prime}, \lambda^{\prime}\right)} \mathcal{V}_{\lambda^{\prime}}^{\dagger}\left(\mathbf{C}^{\prime}\right) / \sim,
$$

where the disjoint union is over all labeled marked Riemann surfaces $\left(\mathbf{C}^{\prime}, \lambda^{\prime}\right)$ discussed above and $\sim$ is the equivalence relation generated by the propagation of vacua isomorphisms given in Theorem 4.3 . the permutations of the order of the marked points and the change of formal coordinate isomorphisms given in Theorem 4.5

By Theorem 4.4, Proposition4.1 and 2, this $\sim$ is also an equivalence relation. We also remark that if we apply definition 4.6 to a labeled marked Riemann surface $(\mathbf{C}, \lambda)$ which is already saturated, then we obtain a space of vacua which is naturally isomorphic to the space of vacua definition 4.5 produces.

\section{The Bundle of Vacua over Teichmüller SPaCe}

5.1. Definition of the sheaf of vacua. Let $\mathfrak{F}=\left(\pi: \mathcal{C} \rightarrow \mathcal{B} ; s_{1}, \ldots, s_{N} ; \eta_{1}, \ldots, \eta_{N}\right)$ be a family of pointed saturated Riemann Surfaces of genus $g$ with formal neighbourhoods.

The sheaf $\widehat{\mathfrak{g}}_{N}(\mathcal{B})$ of affine Lie algebra over $\mathcal{B}$ is the sheaf of $\mathcal{O}_{\mathcal{B}}$-module

$$
\widehat{\mathfrak{g}}_{N}(\mathcal{B})=\mathfrak{g} \otimes_{\mathbb{C}}\left(\bigoplus_{j=1}^{N} \mathcal{O}_{\mathcal{B}}\left(\left(\xi_{j}\right)\right)\right) \oplus \mathcal{O}_{\mathcal{B}} \cdot c
$$

with the following commutation relation, which is $\mathcal{O}_{\mathcal{B}}$-bilinear.

$$
\begin{aligned}
& {\left[\left(X_{1} \otimes f_{1}, \ldots, X_{N} \otimes f_{N}\right),\left(Y_{1} \otimes g_{1}, \ldots, Y_{N} \otimes g_{N}\right)\right]} \\
& \quad=\left(\left[X_{1}, Y_{1}\right] \otimes\left(f_{1} g_{1}\right), \ldots,\left[X_{N}, Y_{N}\right] \otimes\left(f_{N} g_{N}\right)\right) \oplus c \cdot \sum_{j=1}^{N}\left(X_{j}, Y_{j}\right) \underset{\xi_{j}=0}{\operatorname{Res}}\left(g_{j} d f_{j}\right)
\end{aligned}
$$


where $X_{j}, Y_{j} \in \mathfrak{g}, \quad f_{j}, g_{j} \in \mathcal{O}_{\mathcal{B}}\left(\left(\xi_{j}\right)\right)$ and we require $c$ to be central. Put

$$
\widehat{\mathfrak{g}}(\mathfrak{F})=\mathfrak{g} \otimes_{\mathbb{C}} \pi_{*}\left(\mathcal{O}_{\mathcal{C}}(* S)\right)
$$

where we define

$$
\begin{aligned}
S & =\sum_{j=1}^{N} s_{j}(\mathcal{B}) \\
\pi_{*}\left(\mathcal{O}_{\mathcal{C}}(* S)\right) & =\underset{k}{\stackrel{\operatorname{Lim}}{\longleftarrow}} \pi_{*}\left(\mathcal{O}_{\mathcal{C}}(k S)\right) .
\end{aligned}
$$

Laurent expansion using the formal neighbourhoods $\eta_{j}$ 's gives an inclusion:

$$
\tilde{t}: \pi_{*}\left(\mathcal{O}_{\mathcal{B}}(* S)\right) \rightarrow \bigoplus_{j=1}^{N} \mathcal{O}_{\mathcal{B}}\left(\left(\xi_{j}\right)\right)
$$

and we may regard $\widehat{\mathfrak{g}}(\mathfrak{F})$ as a Lie subalgebra of $\widehat{\mathfrak{g}}_{N}(\mathcal{B})$. For any $\vec{\lambda}=\left(\lambda_{1}, \ldots, \lambda_{N}\right) \in\left(P_{\ell}\right)^{N}$, put

$$
\begin{aligned}
& \mathcal{H}_{\vec{\lambda}}(\mathcal{B})=\mathcal{O}_{\mathcal{B}} \otimes_{\mathbb{C}} \mathcal{H}_{\vec{\lambda}}, \\
& \mathcal{H}_{\vec{\lambda}}^{\dagger}(\mathcal{B})=\underline{\operatorname{Hom}}_{\mathcal{O}_{\mathcal{B}}}\left(\mathcal{H}_{\vec{\lambda}}(\mathcal{B}), \mathcal{O}_{\mathcal{B}}\right)=\mathcal{O}_{\mathcal{B}} \otimes_{\mathbb{C}} \mathcal{H}_{\vec{\lambda}}^{\dagger}
\end{aligned}
$$

The pairing (28) induces an $\mathcal{O}_{\mathcal{B}}$-bilinear pairing

$$
\langle\mid\rangle: \mathcal{H}_{\vec{\lambda}}^{\dagger}(\mathcal{B}) \times \mathcal{H}_{\vec{\lambda}}(\mathcal{B}) \rightarrow \mathcal{O}_{\mathcal{B}} .
$$

The sheaf of affine Lie algebra $\widehat{\mathfrak{g}}_{N}(\mathcal{B})$ acts on $\mathcal{H}_{\vec{\lambda}}(\mathcal{B})$ and $\mathcal{H}_{\vec{\lambda}}^{\dagger}(\mathcal{B})$ by

$$
\begin{aligned}
\left(\left(X_{1} \otimes \sum_{n \in \mathbf{Z}} a_{n}^{(1)} \xi_{1}^{n}\right), \ldots,\left(X_{N} \otimes \sum_{n \in \mathbf{Z}} a_{n}^{(N)} \xi_{N}^{n}\right)\right)(F \otimes|\Psi\rangle) \\
=\sum_{j=1}^{N} \sum_{n \in \mathbf{Z}}\left(a_{n}^{(j)} F\right) \otimes \rho_{j}\left(X_{j}(n)\right)|\Psi\rangle
\end{aligned}
$$

The action of $\widehat{\mathfrak{g}}_{N}(\mathcal{B})$ on $\mathcal{H}_{\vec{\lambda}}^{\dagger}(\mathcal{B})$ is the dual action of $\mathcal{H}_{\vec{\lambda}}(\mathcal{B})$, that is,

$$
\langle\Psi a \mid \Phi\rangle=\langle\Psi \mid a \Phi\rangle \quad \text { for any } a \in \widehat{\mathfrak{g}}_{N} .
$$

Definition 5.1. For the family $\mathfrak{F}$ of pointed Riemann surfaces with formal neighbourhoods, we define the sheaves of $\mathcal{O}_{\mathcal{B}}$-modules on $\mathcal{B}$

$$
\begin{aligned}
& \mathcal{V}_{\vec{\lambda}}(\mathfrak{F})=\mathcal{H}_{\vec{\lambda}}(\mathcal{B}) / \widehat{\mathfrak{g}}(\mathfrak{F}) \mathcal{H}_{\vec{\lambda}}(\mathcal{B}) \\
& \mathcal{V}_{\vec{\lambda}}^{\dagger}(\mathfrak{F})=\underline{\operatorname{Hom}}_{\mathcal{O}_{\mathcal{B}}}\left(\mathcal{V}_{\vec{\lambda}}(\mathcal{B}), \mathcal{O}_{\mathcal{B}}\right) .
\end{aligned}
$$

These are the sheaf of covacua and the sheaf of vacua attached to the family $\mathfrak{F}$. 
Note that we have

$$
\mathcal{V}_{\vec{\lambda}}^{\dagger}(\mathfrak{F})=\left\{\left\langle\Psi\left|\in \mathcal{H}_{\vec{\lambda}}^{\dagger}(\mathfrak{F})\right|\langle\Psi| a=0 \quad \text { for any } a \in \widehat{\mathfrak{g}}(\mathfrak{F})\right\}\right.
$$

The pairing (35) ) induces an $\mathcal{O}_{\mathcal{B}}$-bilinear pairing

$$
\langle\mid\rangle: \mathcal{V}_{\vec{\lambda}}^{\dagger}(\mathfrak{F}) \times \mathcal{V}_{\vec{\lambda}}(\mathfrak{F}) \rightarrow \mathcal{O}_{\mathcal{B}}
$$

By Theorem 4.1.6 in [19] and Corollary 4.2.4 in [19] we have the following highly non-trivial theorem. Note that the theorem is true even for a family of nodal curves (see Theorem 14.1).

Theorem 5.1. The sheaves $\mathcal{V}_{\vec{\lambda}}^{\dagger}(\mathfrak{F})$ and $\mathcal{V}_{\vec{\lambda}}(\mathfrak{F})$ are locally free sheaves of $\mathcal{O}_{\mathcal{B}}$-modules of finite rank over $\mathcal{B}$. They are dual to each other.

Hence $\mathcal{V}_{\vec{\lambda}}^{\dagger}(\mathfrak{F})$ is a holomorphic vector bundles over $\mathcal{B}$.

5.2. Properties of the sheaf of vacua. To construct the bundle of vacua over Teichmüller space, we further need the following obvious property of the sheaf of vacua construction.

Lemma 5.1. Let $\mathfrak{F}_{i}$ be two families of saturated pointed Riemann surfaces with formal neighbourhoods over the same base $\mathcal{B}$. Let $\Phi:\left(\mathfrak{F}_{1}, \lambda_{1}\right) \rightarrow\left(\mathfrak{F}_{2}, \lambda_{2}\right)$ be an isomorphism of labeled families, which induces the identity map on the base. Then the identity map on $\mathcal{H}_{\vec{\lambda}}^{\dagger}(\mathcal{B})$ induces a canonical isomorphism

$$
\mathcal{V}^{\dagger}(\Phi): \mathcal{V}_{\vec{\lambda}_{1}}^{\dagger}\left(\mathfrak{F}_{1}\right) \rightarrow \mathcal{V}_{\vec{\lambda}_{2}}^{\dagger}\left(\mathfrak{F}_{2}\right)
$$

Suppose that we have two families of pointed Riemann surfaces with formal neighbourhoods $\mathfrak{F}_{i}$, $i=1,2$ on a pointed saturated and stable surface $(\Sigma, P)$, with the property that they have the same image $\Psi_{\mathfrak{F}_{1}}\left(\mathcal{B}_{1}\right)=\Psi_{\mathfrak{F}_{2}}\left(\mathcal{B}_{2}\right)$ in Teichmüller space $\mathcal{T}_{(\Sigma, P)}$ and that $\mathfrak{F}_{2}$ is a good family. For such a pair of families there exists by Proposition 3.2 a unique fiber preserving biholomorphism $\Phi_{12}: \mathcal{C}_{1} \rightarrow \mathcal{C}_{2}$ covering $\Psi_{\mathfrak{F}_{2}}^{-1} \Psi_{\mathfrak{F}_{1}}$ such that $\Phi_{\mathfrak{F}_{2}}^{-1} \Phi_{12} \Phi_{\mathfrak{F}_{1}}:(Y, P) \rightarrow(Y, P)$ is isotopic to $\Psi_{\mathfrak{F}_{2}}^{-1} \Psi_{\mathfrak{F}_{1}} \times$ Id through such fiber preserving maps inducing the identity on the first order neighbourhood of $P$.

We note that $\left(\Phi_{12}^{*}\left(\vec{\eta}_{2}\right)\right)^{(1)}=\left(S \vec{\eta}_{1}\right)^{(1)}$, where $S$ is some permutation of $\{1, \ldots, N\}$, i.e. $\Phi_{12}^{*}\left(\vec{\eta}_{2}\right)$ induce the same first order formal neighbourhoods as $S \vec{\eta}_{1}$ does. Let $\vec{h}$ be the formal change of coordinates from $S \vec{\eta}_{1}$ to $\Phi_{12}^{*}\left(\vec{\eta}_{2}\right)$. Let $\widetilde{\mathfrak{F}_{1}}=\Phi_{12}^{*}\left(\mathfrak{F}_{2}\right)$. Then $\Phi_{12}$ induces an isomorphism of families from $\widetilde{\mathfrak{F}_{1}}$ to $\left(\Psi_{\mathfrak{F}_{2}}^{-1} \Psi_{\mathfrak{F}_{1}}\right)^{*}\left(\mathfrak{F}_{2}\right)$. Choose labelings $\vec{\lambda}_{1}$ of $\mathfrak{F}_{1}$ and $\vec{\lambda}_{2}$ of $\mathfrak{F}_{2}$, which are compatible under the above isomorphism.

Proposition 5.1. The action of $S$ on $\mathcal{H}_{\vec{\lambda}_{1}}^{\dagger}\left(\mathcal{B}_{1}\right)$ induces an isomorphism from $\mathcal{V}_{\vec{\lambda}_{1}}^{\dagger}\left(\mathfrak{F}_{1}\right)$ to $\mathcal{V}_{\vec{\lambda}_{2}}^{\dagger}\left(S \mathfrak{F}_{1}\right)$, where $S$ acts on the family $\mathfrak{F}_{1}$ by permuting the numbering of the formal neighbourhoods and the sections. Furthermore $G[\vec{h}]: \mathcal{H}_{\vec{\lambda}_{2}}^{\dagger}\left(\mathcal{B}_{1}\right) \rightarrow \mathcal{H}_{\vec{\lambda}_{2}}^{\dagger}\left(\mathcal{B}_{1}\right)$ induces an isomorphism from $\mathcal{V}_{\vec{\lambda}_{2}}^{\dagger}\left(S \mathfrak{F}_{1}\right)$ to $\mathcal{V}_{\vec{\lambda}_{2}}^{\dagger}\left(\widetilde{\mathfrak{F}_{1}}\right)$. The natural pull back isomorphisms provided by Lemma 4.1.3 in [19] and the families isomorphism 
(36) provided by Lemma [5.1] induces an isomorphism from $\mathcal{V}_{\vec{\lambda}_{2}}^{\dagger}\left(\widetilde{\mathfrak{F}_{1}}\right)$ to $\left(\Psi_{\mathfrak{F}_{2}}^{-1} \Psi_{\mathfrak{F}_{1}}\right)^{*} \mathcal{V}_{\vec{\lambda}_{2}}^{\dagger}\left(\mathfrak{F}_{2}\right)$. The composite of these three isomorphism gives the transformation isomorphism

$$
G_{12}: \mathcal{V}_{\vec{\lambda}_{1}}^{\dagger}\left(\mathfrak{F}_{1}\right) \rightarrow\left(\Psi_{\mathfrak{F}_{2}}^{-1} \Psi_{\mathfrak{F}_{1}}\right)^{*} \mathcal{V}_{\vec{\lambda}_{2}}^{\dagger}\left(\mathfrak{F}_{2}\right)
$$

The isomorphisms satisfies the cocycle condition

$$
G_{12}\left(\Psi_{\mathfrak{F}_{2}}^{-1} \Psi_{\mathfrak{F}_{1}}\right)^{*} G_{23}=G_{13} .
$$

Proof. The cocycle condition follows, since $G_{12}$ is induced from the permutation $S$ and the isomorphism $G[\vec{h}]$ on the $\mathcal{H}$-level, combined with formula (2) of Theorem 3.2 .4 in [19].

Let $\mathfrak{F}=\left(\pi: \mathcal{C} \rightarrow \mathcal{B} ; s_{1}, \ldots, s_{N} ; \eta_{1}, \ldots, \eta_{N}\right)$ be a family of pointed Riemann surfaces with formal neighbourhoods. Let $s_{N+1}$ be a further section of $\pi$ disjoint from the $s_{i}$ and $\eta_{N+1}$ a formal neighbourhood along $s_{N+1}$ and set $\widetilde{\mathfrak{F}}=\left(\pi: \mathcal{C} \rightarrow \mathcal{B} ; s_{1}, \ldots, s_{N+1} ; \eta_{1}, \ldots, \eta_{N+1}\right)$

Theorem 5.2. The inclusion

$$
\begin{aligned}
\mathcal{H}_{\vec{\lambda}}(\mathcal{B}) & \longrightarrow \mathcal{H}_{\vec{\lambda}}(\mathcal{B}) \otimes \mathcal{H}_{0}(\mathcal{B}) \\
|v\rangle & \longrightarrow|v\rangle \otimes|0\rangle
\end{aligned}
$$

induces the propagation of vacua isomorphism

$$
P_{\widetilde{F}, \mathfrak{F}}: \mathcal{V}_{\vec{\lambda}, 0}^{\dagger}(\widetilde{\mathfrak{F}}) \rightarrow \mathcal{V}_{\vec{\lambda}}^{\dagger}(\mathfrak{F})
$$

5.3. The definition of the bundle of vacua over Teichmüller space. Let $\Sigma$ be a closed oriented smooth surface and let $P$ be a finite set of marked points on $\Sigma$. Let $\lambda$ be a labeling of $(\Sigma, P)$ and assume $(\Sigma, P)$ is stable and saturated. We now define a holomorphic vector bundle $\mathcal{V}_{\lambda}^{\dagger}=\mathcal{V}_{\lambda}^{\dagger}(\Sigma, P)$ over Teichmüller space $\mathcal{T}_{(\Sigma, P)}$ using the cover $\left\{\Psi_{\mathfrak{F}}(\mathcal{B})\right\}$, where $\mathfrak{F}$ runs over the good families of complex structures on $(\Sigma, P)$.

Definition 5.2. A holomorphic vector bundle $\mathcal{V}_{\lambda}^{\dagger}=\mathcal{V}_{\lambda}^{\dagger}(\Sigma, P)$ over Teichmüller space $\mathcal{T}_{(\Sigma, P)}$ is specify to be the bundle $\left(\Psi_{\mathfrak{F}}^{-1}\right)^{*} \mathcal{V}_{\vec{\lambda}}^{\dagger}(\mathfrak{F})$ over $\Psi_{\mathfrak{F}}(\mathcal{B})$ for any good families of complex structures on $(\Sigma, P)$. On overlaps of the image of two good families, we use the glueing isomorphism (37) to glue the corresponding bundles together.

Proposition 5.1 implies that $\mathcal{V}_{\lambda}^{\dagger}(\Sigma, P)$ is a vector bundle over $\mathcal{T}_{(\Sigma, P)}$ with the following property.

Proposition 5.2. For any stable and saturated family $\tilde{\mathfrak{F}}$ of pointed curves with formal neighbourhoods on $(\Sigma, P)$ we have a preferred isomorphism

$$
\Upsilon_{\tilde{\mathfrak{F}}}: \mathcal{V}_{\vec{\lambda}}^{\dagger}(\tilde{\mathfrak{F}}) \rightarrow \Psi_{\tilde{\mathfrak{F}}}^{*} \mathcal{V}_{\lambda}^{\dagger}(\Sigma, P)
$$

induced by the transformation isomorphism between $\mathcal{V}_{\vec{\lambda}}^{\dagger}(\tilde{\mathfrak{F}})$ and $\mathcal{V}_{\vec{\lambda}}^{\dagger}(\mathfrak{F})$, for good families $\mathfrak{F}$ of complex structures on $(\Sigma, P)$ such that $\Psi_{\mathfrak{F}}(\mathcal{B})$ intersect $\Psi_{\tilde{\mathfrak{F}}}\left(\mathcal{B}^{\prime}\right)$ nonempty. 
Let $(\Sigma, P, \lambda)$ be a general labeled pointed surface, i.e. $(\Sigma, P)$ might not be stable nor saturated. Let $\left(\Sigma, P_{i}, \lambda_{i}\right), i=1,2$ be any labeled marked surfaces obtained from $(\Sigma, P, \lambda)$ by labeling further points by $0 \in P_{\ell}$. Assume that $\left(\Sigma, P_{i}\right)$ are stable and saturated pointed surfaces. Let $\bar{P}=P_{1} \cup P_{2}$ and $\bar{\lambda}$ be the induced labeling of $\bar{P}$. Note that $(\Sigma, \bar{P})$ is also stable and saturated.

We get holomorphic projection maps $\pi_{i}: \mathcal{T}_{(\Sigma, \bar{P})} \rightarrow \mathcal{T}_{\left(\Sigma, P_{i}\right)}$. As a direct consequence of Proposition 4.1 we get the following.

Proposition 5.3. Iterations of the propagation of vacua isomorphism given in Theorem5.2 induces natural isomorphisms of bundles

$$
\mathcal{V}_{\bar{\lambda}}^{\dagger}(\Sigma, \bar{P}) \cong \pi_{1}^{*} \mathcal{V}_{\lambda_{1}}^{\dagger}\left(\Sigma, P_{1}\right) \cong \pi_{2}^{*} \mathcal{V}_{\lambda_{2}}^{\dagger}\left(\Sigma, P_{2}\right)
$$

which satisfies associativity.

Suppose now $f:\left(\Sigma_{1}, P_{1}\right) \rightarrow\left(\Sigma_{2}, P_{2}\right)$ is a morphism of stable and saturated pointed surfaces. Then of course $f$ induces a morphism $f^{*}$ from $\mathcal{T}_{\left(\Sigma_{1}, P_{1}\right)}$ to $\mathcal{T}_{\left(\Sigma_{2}, P_{2}\right)}$. Let $\lambda_{1}$ be a labeling of $\left(\Sigma_{1}, P_{1}\right)$ and let $\lambda_{2}$ be the induced labeling on $\left(\Sigma_{2}, P_{2}\right)$ such that $f:\left(\Sigma_{1}, P_{1}, \lambda_{1}\right) \rightarrow\left(\Sigma_{2}, P_{2}, \lambda_{2}\right)$ is a morphism of labeled pointed surfaces. Let now $\mathfrak{F}_{1}$ be a good family of stable pointed curves with formal neighbourhoods of $\left(\Sigma_{1}, P_{1}\right)$. Then by composing with $f^{-1} \times$ Id we get a good family $\mathfrak{F}_{2}$ of stable pointed curves with formal neighbourhoods on $\left(\Sigma_{2}, P_{2}\right)$ over the same base $\mathcal{B}_{1}$. The identity morphism on $\mathcal{H}_{\vec{\lambda}}^{\dagger}\left(\mathcal{B}_{1}\right)$ then induces a morphism $\mathcal{V}^{\dagger}(f): \mathcal{V}_{\vec{\lambda}}^{\dagger}\left(\mathfrak{F}_{1}\right) \rightarrow \mathcal{V}_{\vec{\lambda}}^{\dagger}\left(\mathfrak{F}_{2}\right)$ which covers the identity on the base. This is precisely the morphism induced from the morphism of families $\Phi_{f}=f \times \operatorname{Id}: \mathfrak{F}_{1} \rightarrow \mathfrak{F}_{2}$ by Lemma 5.1. This intern then induces a morphism $\mathcal{V}^{\dagger}(f):\left(\Psi_{\mathfrak{F}_{1}}^{-1}\right)^{*}\left(\mathcal{V}_{\vec{\lambda}_{1}}^{\dagger}\left(\mathfrak{F}_{1}\right)\right) \rightarrow\left(\Psi_{\mathfrak{F}_{2}}^{-1}\right)^{*}\left(\mathcal{V}_{\vec{\lambda}_{2}}^{\dagger}\left(\mathfrak{F}_{2}\right)\right)$ which covers $f^{*}: \Psi_{\mathfrak{F}_{1}}\left(B_{1}\right) \rightarrow \Psi_{\mathfrak{F}_{2}}\left(B_{1}\right)$

Proposition 5.4. The above construction provides a well defined lift of $f^{*}: \mathcal{T}_{\left(\Sigma_{2}, P_{2}\right)} \rightarrow \mathcal{T}_{\left(\Sigma_{1}, P_{1}\right)}$ to a morphism $\mathcal{V}^{\dagger}(f): \mathcal{V}_{\lambda_{1}}^{\dagger}\left(\Sigma_{1}, P_{1}\right) \rightarrow \mathcal{V}_{\lambda_{2}}^{\dagger}\left(\Sigma_{2}, P_{2}\right)$ which behaves well under compositions.

Proof. Let $f^{\prime}$ be a diffeomorphism whose first order neighbourhood from $P_{1}$ to $P_{2}$ is the same as $f$ 's and such that $f^{\prime}$ is isotopic to $f$ among such. Let $\mathfrak{F}_{2^{\prime}}$ be obtained from $\mathfrak{F}_{1}$ by composing with $\left(f^{\prime}\right)^{-1} \times$ Id. Then

$$
\Phi=\Phi_{\mathfrak{F}_{1}} \circ\left(\left(\left(f^{\prime}\right)^{-1} \circ f\right) \times \mathrm{Id}\right) \circ \Phi_{\mathfrak{F}_{1}}^{-1}: \mathcal{C}_{1} \rightarrow \mathcal{C}_{1}
$$

is the unique biholomorphism from Proposition 3.2 . Hence we get a commutative diagram

$$
\begin{array}{cc}
\left(\Psi_{\mathfrak{F}_{1}}\right)^{*}\left(\mathcal{V}_{\vec{\lambda}_{1}}^{\dagger}\left(\mathfrak{F}_{1}\right)\right) \stackrel{\mathcal{V}^{\dagger}(f)}{\longrightarrow} & \left(\Psi_{\mathfrak{F}_{2}}\right)^{*}\left(\mathcal{V}_{\vec{\lambda}_{2}}^{\dagger}\left(\mathfrak{F}_{2}\right)\right) \\
=\downarrow & \downarrow G_{22^{\prime}} \\
\left(\Psi_{\mathfrak{F}_{1}}\right)^{*}\left(\mathcal{V}_{\vec{\lambda}_{1}}^{\dagger}\left(\mathfrak{F}_{1}\right)\right) \stackrel{\mathcal{V}^{\dagger}\left(f^{\prime}\right)}{\longrightarrow} & \left(\Psi_{\mathfrak{F}_{2^{\prime}}}\right)^{*}\left(\mathcal{V}_{\vec{\lambda}_{2}}^{\dagger}\left(\mathfrak{F}_{2^{\prime}}\right)\right)
\end{array}
$$

which shows that $\mathcal{V}^{\dagger}(f): \mathcal{V}_{\vec{\lambda}_{1}}^{\dagger}\left(\Sigma_{1}, P_{1}\right) \rightarrow \mathcal{V}_{\vec{\lambda}_{2}}^{\dagger}\left(\Sigma_{2}, P_{2}\right)$ is well defined. It is obvious that $\mathcal{V}^{\dagger}(f g)=$ $\mathcal{V}^{\dagger}(f) \mathcal{V}^{\dagger}(g)$. 
Assume that $\left(\Sigma_{i}, P_{i}, \lambda_{i}\right)$ are labeled pointed surfaces, which need not be neither stable nor saturated. Let $f:\left(\Sigma_{1}, P_{1}, \lambda_{1}\right) \rightarrow\left(\Sigma_{2}, P_{2}, \lambda_{2}\right)$ be an orientation preserving diffeomorphism of labeled pointed surfaces. Let $\left(\Sigma_{i}, P_{i}^{\prime}, \lambda_{i}^{\prime}\right)$ be labeled pointed surfaces obtained from $\left(\Sigma_{i}, P_{i}, \lambda_{i}\right)$ by labeling further points by $0 \in P_{\ell}$ such that $\left(\Sigma_{i}, P_{i}^{\prime}, \lambda_{i}^{\prime}\right)$ are stable and saturated labeled pointed surfaces such that $f:\left(\Sigma_{1}, P_{1}^{\prime}, \lambda_{1}^{\prime}\right) \rightarrow\left(\Sigma_{2}, P_{2}^{\prime}, \lambda_{2}^{\prime}\right)$ is a morphism of labeled pointed surfaces. We obviously have the following result.

Proposition 5.5. The lift of $f^{*}: \mathcal{T}_{\left(\Sigma_{1}, P_{1}^{\prime}\right)} \rightarrow \mathcal{T}_{\left(\Sigma_{2}, P_{2}^{\prime}\right)}$ to a morphism $\mathcal{V}^{\dagger}(f): \mathcal{V}_{\lambda_{1}^{\prime}}^{\dagger}\left(\Sigma_{1}, P_{1}^{\prime}\right) \rightarrow \mathcal{V}_{\lambda_{2}^{\prime}}^{\dagger}\left(\Sigma_{2}, P_{2}^{\prime}\right)$ as given by Theorem 5.4 is compatible with the isomorphisms given in Proposition 5.3.

\section{The Connection in the Bundle of Vacua over Teichmüller SPace}

6.1. Twisted first order differential operators acting on the sheaf of vacua. Let $\mathfrak{F}=(\pi$ : $\left.\mathcal{C} \rightarrow \mathcal{B} ; s_{1}, \ldots, s_{N} ; \eta_{1}, \ldots, \eta_{N}\right)$ is a family of saturated pointed Riemann surfaces of genus $g$ with formal neighbourhoods. We will assume that $\mathfrak{F}^{(0)}=\left(\pi: \mathcal{C} \rightarrow \mathcal{B} ; s_{1}, \ldots, s_{N}\right)$ be a versal family of pointed stable curves of genus $g$ in the sense of definition 1.2.2. in [19. We consider the divisors

$$
S_{j}=s_{j}(\mathcal{B}), \quad S=\sum_{j=1}^{N} S_{j} .
$$

There is an exact sequence

$$
0 \rightarrow \Theta_{\mathcal{C} / \mathcal{B}} \rightarrow \Theta_{\mathcal{C}} \stackrel{d \pi}{\rightarrow} \pi^{*} \Theta_{\mathcal{B}} \rightarrow 0
$$

where $\Theta_{\mathcal{C} / \mathcal{B}}$ is a sheaf of vector fields tangent to the fibres of $\pi$. Put

$$
\Theta_{\mathcal{C}, \pi}^{\prime}=d \pi^{-1}\left(\pi^{-1} \Theta_{\mathcal{B}}\right) .
$$

Hence, $\Theta_{\mathcal{C}, \pi}^{\prime}$ is a sheaf of vector field on $\mathcal{C}$ whose vertical components are constant along the fibers of $\pi$. That is, in a neighbourhood of a point of a fiber $\Theta_{\mathcal{C}, \pi}^{\prime}$ consists of germs of holomorphic vector fields of the form

$$
a(z, u) \frac{\partial}{\partial z}+\sum_{i=1}^{n} b_{i}(u) \frac{\partial}{\partial u_{i}}
$$

where $\left(z, u_{1}, \ldots, u_{n}\right)$ is a system of local coordinates such that the mapping $\pi$ is expressed as the projection

$$
\pi\left(z, u_{1}, \ldots, u_{n}\right)=\left(u_{1}, \ldots, u_{n}\right) .
$$

More generally, we can define a sheaf $\Theta_{\mathcal{C}}^{\prime}(m S)_{\pi}$ as the one consisting of germs of meromorphic vector fields of the form

$$
A(z, u) \frac{\partial}{\partial z}+\sum_{i=1}^{n} B_{i}(u) \frac{\partial}{\partial u_{i}}
$$

where $A(z, u)$ has the poles of order at most $m$ along $S$. 
We have an exact sequence

$$
0 \rightarrow \Theta_{\mathcal{C} / \mathcal{B}}(m S) \rightarrow \Theta_{\mathcal{C}}^{\prime}(m S)_{\pi} \stackrel{d \pi}{\rightarrow} \pi^{-1} \Theta_{\mathcal{B}} \rightarrow 0
$$

Note that $\Theta_{\mathcal{C}}^{\prime}(m S)_{\pi}$ has the structure of a sheaf of Lie algebras by the usual bracket operation on vector fields and the above exact sequence is one of sheaves of Lie algebras.

For $m>\frac{1}{N}(2 g-2)$ we have an exact sequence of $\mathcal{O}_{\mathcal{B}}$-modules.

$$
0 \rightarrow \pi_{*} \Theta_{\mathcal{C} / \mathcal{B}}(m S) \rightarrow \pi_{*} \Theta_{\mathcal{C}}^{\prime}(m S)_{\pi} \stackrel{d \pi}{\rightarrow} \Theta_{\mathcal{B}} \rightarrow 0
$$

which is also an exact sequence of sheaves of Lie algebras. Taking $m \rightarrow \infty$ we obtain the exact sequence

$$
0 \rightarrow \pi_{*} \Theta_{\mathcal{C} / \mathcal{B}}(* S) \rightarrow \pi_{*} \Theta_{\mathcal{C}}^{\prime}(* S)_{\pi} \stackrel{d \pi}{\rightarrow} \Theta_{\mathcal{B}} \rightarrow 0 .
$$

Recall that we have the following exact sequence of $\mathcal{O}_{\mathcal{B}}$-modules.

$$
\left.\left.0 \rightarrow \Theta_{\mathcal{C} / \mathcal{B}}(-S)\right) \rightarrow \Theta_{\mathcal{C} / \mathcal{B}}(m S)\right) \rightarrow \bigoplus_{j=1}^{N} \bigoplus_{k=0}^{m} \mathcal{O}_{\mathcal{B}} \xi_{j}^{-k} \frac{d}{d \xi_{j}} \rightarrow 0
$$

Which for any positive integer $m \geq 4 g-3$ gives the following exact sequence

$$
0 \rightarrow \pi_{*}\left(\Theta_{\mathcal{C} / \mathcal{B}}(m S)\right) \stackrel{b_{m}}{\rightarrow} \bigoplus_{j=1}^{N} \bigoplus_{k=0}^{m} \mathcal{O}_{\mathcal{B}} \xi_{j}^{-k} \frac{d}{d \xi_{j}} \stackrel{\vartheta_{m}}{\rightarrow} R^{1} \pi_{*} \Theta_{\mathcal{C} / \mathcal{B}}(-S) \rightarrow 0
$$

From which we deduce the following exact sequence of $\mathcal{O}_{\mathcal{B}}$-modules

$$
0 \rightarrow \pi_{*}\left(\Theta_{\mathcal{C} / \mathcal{B}}(* S)\right) \stackrel{b}{\rightarrow} \bigoplus_{j=1}^{N} \mathcal{O}_{\mathcal{B}}\left[\xi_{j}^{-1}\right] \frac{d}{d \xi_{j}} \stackrel{\vartheta}{\rightarrow} R^{1} \pi_{*} \Theta_{\mathcal{C} / \mathcal{B}}(-S) \rightarrow 0 .
$$

Note that the mappings $b$ and $b_{m}$ correspond to the Laurent expansions with respect to $\xi_{j}$ up to zero-th order.

By the Kodaira-Spencer theory (see [19] for details) we have the following commutative diagram.

$$
\begin{aligned}
& 0 \rightarrow \pi_{*} \Theta_{\mathcal{C} / \mathcal{B}}(* S) \quad \rightarrow \quad \pi_{*} \Theta_{\mathcal{C}}^{\prime}(* S)_{\pi} \quad \stackrel{d \pi}{\rightarrow} \quad \Theta_{\mathcal{B}} \quad \rightarrow 0 \\
& \| \quad \downarrow p \quad \downarrow \rho \\
& 0 \rightarrow \pi_{*} \Theta_{\mathcal{C} / \mathcal{B}}(* S) \quad \rightarrow \quad \bigoplus_{j=1}^{N} \mathcal{O}_{\mathcal{B}}\left[\xi_{j}^{-1}\right] \frac{d}{d \xi_{j}} \quad \stackrel{\vartheta}{\rightarrow} \quad R^{1} \pi_{*} \Theta_{\mathcal{C} / \mathcal{B}}(-S) \quad \rightarrow 0
\end{aligned}
$$

where $\rho$ is the Kodaira-Spencer mapping of the family $\mathfrak{F}^{(0)}$ and $p$ is given by taking the non-positive part of the $\frac{d}{d \xi_{j}}$ part of the Laurent expansions of the vector fields in $\pi_{*} \Theta_{\mathcal{C}}(m S)_{\pi}$ at $s_{j}(\mathcal{B})$. Since our 
family $\mathfrak{F}^{(0)}$ is versal, it follows from Proposition 1.2.6. in [19, that the Kodaira-Spencer mapping $\rho$ is an isomorphism of $\mathcal{O}_{\mathcal{B}}$-modules. Therefore, $p$ is an isomorphism. Put

$$
\mathcal{L}(\mathfrak{F}):=\bigoplus_{j=1}^{N} \mathcal{O}_{\mathcal{B}}\left[\xi_{j}^{-1}\right] \frac{d}{d \xi_{j}} .
$$

Then, we have the following exact sequence

$$
0 \rightarrow \pi_{*} \Theta_{\mathcal{C} / \mathcal{B}}(* S) \rightarrow \mathcal{L}(\mathfrak{F}) \stackrel{\theta}{\rightarrow} \Theta_{\mathcal{B}} \rightarrow 0
$$

of $\mathcal{O}_{\mathcal{B}}$-modules. The Lie bracket [ , $]_{d}$ on $\mathcal{L}(\mathfrak{F})$ is determined by requiring $p$ to be a Lie algebra isomorphism. Thus, for $\vec{\ell}, \vec{m} \in \mathcal{L}(\mathfrak{F})$ we have

$$
[\vec{\ell}, \vec{m}]_{d}=[\vec{\ell}, \vec{m}]_{0}+\theta(\vec{\ell})(\vec{m})-\theta(\vec{m})(\vec{\ell})
$$

where $[, \quad]_{0}$ is the usual bracket of formal vector fields and the action of $\theta(\vec{\ell})$ on

$$
\vec{m}=\left(m_{1} \frac{d}{d \xi_{1}}, \ldots, m_{N} \frac{d}{d \xi_{N}}\right)
$$

is defined by

$$
\theta(\vec{\ell})(\vec{m})=\left(\theta(\vec{\ell})\left(m_{1}\right) \frac{d}{d \xi_{1}}, \ldots, \theta(\vec{\ell})\left(m_{N}\right) \frac{d}{d \xi_{N}}\right) .
$$

Then, the exact sequence (38) is an exact sequence of sheaves of Lie algebras.

For $\vec{\ell}=\left(\underline{l}_{1}, \ldots, \underline{l}_{N}\right) \in \mathcal{L}(\mathfrak{F})$, the action $D(\vec{\ell})$ on $\mathcal{H}_{\vec{\lambda}}(\mathcal{B})$ is defined by

$$
D(\vec{\ell})(F \otimes|\Phi\rangle)=\theta(\vec{\ell})(F) \otimes|\Phi\rangle-F \cdot\left(\sum_{j=1}^{N} \rho_{j}\left(T\left[\underline{l}_{j}\right]\right)|\Phi\rangle\right.
$$

where

$$
F \in \mathcal{O}_{\mathcal{B}}, \quad|\Phi\rangle \in \mathcal{H}_{\vec{\lambda}}
$$

We have the following propositions.

Proposition 6.1 ([19, Proposition 4.2.2]). The action $D(\vec{\ell})$ of $\vec{\ell} \in \mathcal{L}(\mathfrak{F})$ on $\mathcal{H}_{\vec{\lambda}}(\mathcal{B})$ defined above has the following properties.

1) For any $f \in \mathcal{O}_{\mathcal{B}}$ we have

$$
D(f \vec{\ell})=f D(\vec{\ell})
$$

2) For $\vec{\ell}, \vec{m} \in \mathcal{L}(\mathfrak{F})$ we have

$$
[D(\vec{\ell}), D(\vec{m})]=D\left([\vec{\ell}, \vec{m}]_{d}\right)+\frac{c_{v}}{12} \sum_{j=1}^{N} \operatorname{Res}_{\xi_{j}=0}\left(\frac{d^{3} \ell_{j}}{d \xi_{j}^{3}} m_{j} d \xi_{j}\right) \cdot i d .
$$

3) For $f \in \mathcal{O}_{\mathcal{B}}$ and $|\phi\rangle \in \mathcal{H}_{\vec{\lambda}}(\mathcal{B})$ we have

$$
D(\vec{\ell})(f|\phi\rangle)=(\theta(\vec{\ell})(f))|\phi\rangle+f D(\vec{\ell})|\phi\rangle .
$$

Namely, $D(\vec{\ell})$ is a first order differential operator, if $\theta(\vec{\ell}) \neq 0$. 
We define the dual action of $\mathcal{L}(\mathfrak{F})$ on $\mathcal{H}_{\vec{\lambda}}^{\dagger}(\mathcal{B})$ by

$$
D(\vec{\ell})(F \otimes\langle\Psi|)=(\theta(\vec{\ell}) F) \otimes\langle\Psi|+\sum_{j=1}^{N} F \cdot\langle\Psi| \rho_{j}\left(T\left[\underline{l}_{j}\right]\right) .
$$

where

$$
F \in \mathcal{O}_{\mathcal{B}}, \quad\langle\Psi| \in \mathcal{H}_{\vec{\lambda}}^{\dagger}(\mathcal{B}) .
$$

Then, for any $|\widetilde{\Phi}\rangle \in \mathcal{H}_{\vec{\lambda}}(\mathcal{B})$ and $\langle\widetilde{\Psi}| \in \mathcal{H}_{\vec{\lambda}}^{\dagger}(\mathcal{B})$, we have

$$
\{D(\vec{\ell})\langle\widetilde{\Psi}|\}|\widetilde{\Phi}\rangle+\langle\widetilde{\Psi}|\{D(\vec{\ell})|\widetilde{\Phi}\rangle\}=\theta(\vec{\ell})\langle\widetilde{\Psi} \mid \widetilde{\Phi}\rangle .
$$

Proposition 6.2 ([19, Proposition 4.2.3]). For any $\vec{\ell} \in \mathcal{L}(\mathfrak{F})$ we have

$$
D(\vec{\ell})\left(\widehat{\mathfrak{g}}(\mathfrak{F}) \mathcal{H}_{\vec{\lambda}}(\mathcal{B})\right) \subset \widehat{\mathfrak{g}}(\mathfrak{F}) \mathcal{H}_{\vec{\lambda}}(\mathcal{B}) .
$$

Hence, $D(\vec{\ell})$ operates on $\mathcal{V}_{\vec{\lambda}}(\mathfrak{F})$. Moreover, it is a first order differential operator, if $\theta(\vec{\ell}) \neq 0$.

Proposition 6.3 ([19, Proposition 4.2.8]). For each element $\vec{\ell} \in \mathcal{L}(\mathfrak{F}), D(\vec{\ell})$ acts on $\mathcal{V}_{\vec{\lambda}}^{\dagger}(\mathfrak{F})$. Moreover, if $\theta(\vec{\ell}) \neq 0$, then $D(\vec{\ell})$ acts on $\mathcal{V}_{\vec{\lambda}}^{\dagger}(\mathfrak{F})$ as a first order differential operator.

Note that for the natural bilinear pairing $\langle\mid\rangle: \mathcal{V}_{\vec{\lambda}}^{\dagger}(\mathfrak{F}) \times \mathcal{V}_{\vec{\lambda}}(\mathfrak{F}) \rightarrow \mathcal{O}_{\mathcal{B}}$, we have the equality

$$
\{D(\vec{\ell})\langle\Psi|\}|\Phi\rangle+\langle\Psi|\{D(\vec{\ell})|\Phi\rangle\}=\theta(\vec{\ell})(\langle\Psi \mid \Phi\rangle) .
$$

6.2. The projectively flat connections on the on the bundle of vacua. Let $\Sigma$ be a closed oriented smooth surface and let $P$ be a finite set of marked points on $\Sigma$. Assume that $(\Sigma, P)$ is stable and saturated pointed surface.

Let $\mathfrak{F}=(\pi: \mathcal{C} \rightarrow \mathcal{B} ; \vec{s} ; \vec{\eta})$ be a family of pointed Riemann Surfaces with formal neighbourhoods on $(\Sigma, P)$. Recall the discussion of symmetric bidifferentials from section 1.4 in [19]. We now introduce the notion of a normalized symmetric bidifferential.

Definition 6.1. A symmetric bidifferential $\omega \in H^{0}\left(\mathcal{C} \times{ }_{\mathcal{B}} \mathcal{C}, \omega_{\mathcal{C} \times{ }_{\mathcal{B}} \mathcal{C} / \mathcal{B}}(2 \Delta)\right)$ with

$$
\omega=\left(\frac{1}{(x-y)^{2}}+\text { holomorphic }\right) d x d y
$$

in a neighbourhood of the diagonal of $\mathcal{C} \times{ }_{\mathcal{B}} \mathcal{C}$ is called a normalized symmetric bidifferential for the family $\mathfrak{F}$.

For a Riemann surface $R$ we let $(\vec{\alpha}, \vec{\beta})=\left(\alpha_{1}, \ldots, \alpha_{g}, \beta_{1}, \ldots, \beta_{g}\right)$ be a symplectic basis of $H_{1}(R, \mathbb{Z})$. We can find a basis $\left\{\omega_{1}, \ldots, \omega_{g}\right\}$ of holomorphic one forms of $R$ with

$$
\int_{\beta_{i}} \omega_{j}=\delta_{i j}, \quad 1 \leq i, j \leq g
$$

The matrix

$$
\tau=\left(\tau_{i j}\right), \quad \tau_{i j}=\int_{\alpha_{i}} \omega_{j}
$$


is then called the period matrix of the Riemann surface $R$. The complex torus

$$
J(R)=\mathbb{C}^{g} /\left(\tau, I_{g}\right)
$$

is called a Jacobian variety. If we chose a point $P$ on $R$ we can define a holomorphic mapping

$$
\begin{aligned}
j: R & \rightarrow J(R) \\
Q & \mapsto\left(\int_{P}^{Q} \omega_{1}, \ldots, \int_{P}^{Q} \omega_{g}\right) .
\end{aligned}
$$

If a family of pointed Riemann surfaces is given, we can construct a family of Jacobian varieties and a family of holomorphic mappings.

We have the following lemma as a consequence of the construction in Section 1.4 in [19].

Lemma 6.1. For any family of pointed Riemann surfaces with formal neighbourhoods $\mathfrak{F}$ on $(\Sigma, P)$ and any symplectic basis $(\vec{\alpha}, \vec{\beta})=\left(\alpha_{1}, \ldots, \alpha_{g}, \beta_{1}, \ldots, \beta_{g}\right)$ of $H_{1}(\Sigma, \mathbb{Z})$, there is a unique normalized symmetric bidifferential $\omega \in H^{0}\left(\mathcal{C} \times{ }_{\mathcal{B}} \mathcal{C}, \omega_{\mathcal{C} \times{ }_{\mathcal{B}} \mathcal{C} / \mathcal{B}}(2 \Delta)\right)$ determined by formula

$$
\omega(x, y) d x d y=\frac{\partial^{2} \log E(x, y)}{\partial x \partial y} d x d y
$$

where $E(x, y)(\sqrt{d x})^{-1}(\sqrt{d y})^{-1}$ is the prime form associated to the symplectic basis $(\vec{\alpha}, \vec{\beta})$ for each Riemann surface.

For a prime form see Chapter II of [9]. Please do note that $\alpha$ and $\beta$ play the reverse roles in [19], but the same as in [2].

Note that a prime form of a Riemann surface $R$ (hence, also a normalized symmetric bidifferential) is uniquely determined by a symplectic basis $(\vec{\alpha}, \vec{\beta})$ of $H_{1}(R, \mathbb{Z})$. If $(\overrightarrow{\widehat{\alpha}}, \overrightarrow{\widehat{\beta}})=\left(\widehat{\alpha}_{1}, \ldots, \widehat{\alpha}_{g}, \widehat{\beta}_{1}, \ldots, \widehat{\beta}_{g}\right)$ is another symplectic basis, there exists a symplectic matrix

$$
\Lambda=\left(\begin{array}{ll}
A & B \\
C & D
\end{array}\right) \in S p(g, \mathbb{Z})
$$

such that

$$
\left(\begin{array}{c}
\widehat{\alpha}_{1} \\
\vdots \\
\widehat{\alpha}_{g} \\
\widehat{\beta}_{1} \\
\vdots \\
\widehat{\beta}_{g}
\end{array}\right)=\left(\begin{array}{cc}
A & B \\
C & D
\end{array}\right)\left(\begin{array}{c}
\alpha_{1} \\
\vdots \\
\alpha_{g} \\
\beta_{1} \\
\vdots \\
\beta_{g}
\end{array}\right) .
$$

Also for any element $\Lambda \in S p(g, \mathbb{Z})$, by (43) we can define a new symplectic basis $\Lambda(\vec{\alpha}, \vec{\beta})=$ $\left(\widehat{\alpha}_{1}, \ldots, \widehat{\alpha}_{g}, \widehat{\beta}_{1}, \ldots, \widehat{\beta}_{g}\right)$. Then the normalized symmetric bidifferential $\widehat{\omega}(x, y) d x d y$ associated to the 
symplectic basis $\Lambda(\vec{\alpha}, \vec{\beta})$ and the normalized symmetric bidifferential $\omega(x, y) d x d y$ have a relation:

$$
\begin{aligned}
\widehat{\omega}(x, y) d x d y= & \omega(x, y) d x d y \\
& -\frac{1}{2} \sum_{i \leq j}\left\{v_{i}(x) v_{j}(y)+v_{j}(x) v_{i}(y)\right\} \frac{\partial}{\partial \tau_{i j}} \log \operatorname{det}(C \tau+D)
\end{aligned}
$$

where $\left\{\omega_{1}=v_{1}(x) d x, \ldots, \omega_{g}=v_{g}(x) d x\right\}$ is a basis of holomorphic one-forms on the Riemann surface $R$ which satisfies (40), $\tau_{i j}$ is defined by (41) and

$$
\Lambda=\left(\begin{array}{ll}
A & B \\
C & D
\end{array}\right) \text {. }
$$

For details see [9], Chapter II.

Let $\omega$ be a normalized symmetric bidifferential for $\mathfrak{F}$. In the formal neighbourhood $\eta_{j}$ we define the quadratic differential (projective connection)

$$
S_{\omega} d \eta_{j}^{2}=6 \lim _{\substack{\eta \rightarrow \eta_{j} \\ \xi \rightarrow \eta_{j}}}\left(\omega(\eta, \xi)-\frac{d \eta d \xi}{(\eta-\xi)^{2}}\right) .
$$

We define

$$
a_{\omega}: \mathcal{L}(\mathfrak{F}) \rightarrow \mathcal{O}_{\mathcal{B}}
$$

as an $\mathcal{O}_{\mathcal{B}}$-module homomorphism by

$$
a_{\omega}(\vec{\ell})=-\frac{c_{v}}{12} \sum_{j=1}^{N} \operatorname{Res}_{\eta_{j}=0}\left(\ell_{j}\left(\eta_{j}\right) S_{\omega}\left(\eta_{j}\right) d \eta_{j}\right)
$$

for all $\vec{\ell} \in \mathcal{L}(\mathfrak{F})$.

Definition 6.2. For each $b \in \mathcal{B}$ and each element $X \in\left(\Theta_{\mathcal{B}}\right)_{b}$, there is an element $\vec{\ell} \in \mathcal{L}(\mathfrak{F})_{b}$ with $\theta(\vec{\ell})=X$. Define an operator $\nabla_{X}^{(\omega)}$ acting on $\mathcal{V}_{\vec{\lambda}}^{\dagger}(\mathfrak{F})_{b}$ from the left by

$$
\nabla_{X}^{(\omega)}(\langle\Phi|)=D(\vec{\ell})\left([\langle\Phi|)+a_{\omega}(\vec{\ell})([\langle\Phi|) .\right.
$$

Theorem 6.1. $\nabla^{(\omega)}$ is a well-defined holomorphic connection in $\mathcal{V}_{\vec{\lambda}}^{\dagger}(\mathfrak{F})$.

In [19] we introduced the holomorphic connection on $\mathcal{V}_{\vec{\lambda}}(\mathfrak{F})_{b}$ and our connection $\nabla^{(\omega)}$ is its dual connection (see Proposition 5.1.4 in [19]).

Definition 6.3. We define a connection in the vector bundle $\mathcal{V}_{\vec{\lambda}}^{\dagger}(\mathfrak{F})$ over $\mathcal{B}$ by letting its $(1,0)$ part be given by the holomorphic connection $\nabla^{(\omega)}$ just defined and its $(0,1)$-part be given by the $\bar{\partial}$-operator determined by the holomorphic structure on $\mathcal{V}_{\vec{\lambda}}^{\dagger}(\mathfrak{F})$. We also denote this connection $\nabla^{(\omega)}$. 
Theorem 6.2. The curvature of the connection $\nabla^{(\omega)}$ is given by

$$
R^{\omega}(X, Y)=\left\{-a_{\omega}(\vec{n})+X\left(a_{\omega}(\vec{m})\right)-Y\left(a_{\omega}(\vec{l})\right)-\frac{c_{v}}{12} \sum_{j=1}^{N} \operatorname{Res}_{\xi_{j}=0}\left(\frac{d^{3} l_{j}}{d \xi_{j}^{3}} m_{j} d \xi_{j}\right)\right\} \otimes \mathrm{Id}
$$

where $\vec{\ell}$ and $\vec{m}$ are liftings of $X$ and $Y$ to $\mathcal{L}(\mathfrak{F})$, i.e. $\theta(\vec{\ell})=X$ and $\theta(\vec{m})=Y$, and $\vec{n}=[\vec{l}, \vec{m}]_{d}($ see (39)). Hence we see that the connection is projectively flat and the curvature is of type $(2,0)$.

Proof. It follows from the definition of the connection in the above definition that the $(1,1)$ and $(0,2)$-part of the curvature vanishes.

6.3. The definition of the connection in the bundle of vacua over Teichmüller space. Suppose we have two good families $\mathfrak{F}_{i}, i=1,2$ of pointed Riemann Surfaces with for mal neighbourhoods, with the property that they have the same image $\Psi_{\mathfrak{F}_{1}}\left(\mathcal{B}_{1}\right)=\Psi_{\mathfrak{F}_{2}}\left(\mathcal{B}_{2}\right)$ in Teichmüller space $\mathcal{T}_{(\Sigma, P)}$.

Lemma 6.2. Let $\nabla_{i}^{(\omega)}$ be the connection in $\mathcal{V}_{\vec{\lambda}}^{\dagger}\left(\mathfrak{F}_{i}\right)$. Then we have that

$$
G_{12}^{*}\left(\nabla_{2}^{(\omega)}\right)=\nabla_{1}^{(\omega)}
$$

Proof. Since the connection is descended from the $\mathcal{H}$-level and $G_{12}$ is also descended from this level, we just need to check the transformation rule on this level. Up on the $\mathcal{H}$-level it follows straight from Theorem $4.4(3)$.

Theorem 6.3. Let $(\Sigma, P, \lambda)$ be a closed oriented stable and saturated marked surface and let $(\vec{\alpha}, \vec{\beta})=$ $\left(\alpha_{1}, \ldots, \alpha_{g}, \beta_{1}, \ldots, \beta_{g}\right)$ be a symplectic basis of $H_{1}(\Sigma, \mathbb{Z})$. There is a unique connection $\nabla^{(\vec{\alpha}, \vec{\beta})}=$ $\nabla^{(\vec{\alpha}, \vec{\beta})}(\Sigma, P)$ in the bundle $\mathcal{V}_{\lambda}^{\dagger}(\Sigma, P)$ over $\mathcal{T}_{(\Sigma, P)}$ with the property that for any good family $\mathfrak{F}$ of stable pointed Riemann surfaces with formal neighbourhoods on $(\Sigma, P)$ we have that

$$
\Psi_{\mathfrak{F}}^{*}\left(\nabla^{(\vec{\alpha}, \vec{\beta})}\right)=\nabla^{(\omega)} .
$$

In particular the connection is holomorphic and projectively flat with $(2,0)$-curvature as described in Theorem 6.2. If we act on the symplectic basis $(\vec{\alpha}, \vec{\beta})$ by an element $\Lambda=\left(\begin{array}{cc}A & B \\ C & D\end{array}\right) \in S p(g, \mathbb{Z})$ by (43), then we have

$$
\nabla^{\Lambda(\vec{\alpha}, \vec{\beta})}-\nabla^{(\vec{\alpha}, \vec{\beta})}=-\frac{c_{v}}{2} \Pi^{*}(d \log \operatorname{det}(C \tau+D)),
$$

where $\Pi$ is the period mapping of holomorphic one-forms form the base space of $\mathfrak{F}$ to the Siegel upperhalf plane of degree $g$. If $f:\left(\Sigma_{1}, P_{1}, \lambda_{1}\right) \rightarrow\left(\Sigma_{2}, P_{2}, \lambda_{1}\right)$ is an orientation preserving diffeomorphism 
of labeled pointed surfaces which maps the symplectic basis $\left(\vec{\alpha}^{(1)}, \vec{\beta}^{(1)}\right)$ of $H_{1}\left(\Sigma_{1}, \mathbb{Z}\right)$ to the symplectic basis $\left(\vec{\alpha}^{(2)}, \vec{\beta}^{(2)}\right)$ of $H_{1}\left(\Sigma_{2}, \mathbb{Z}\right)$ then we have that

$$
\mathcal{V}^{\dagger}(f)^{*}\left(\nabla^{\left(\vec{\alpha}^{(2)}, \vec{\beta}^{(2)}\right)}\right)=\nabla^{\left(\vec{\alpha}^{(1)}, \vec{\beta}^{(1)}\right)} .
$$

Proof. The existence of the connection is a consequence of Lemma 6.2. The transformation law (46) is proved in section 5.2 in [19].

Proposition 6.4. For any stable and saturated family $\tilde{\mathfrak{F}}$ of pointed Riemann surfaces with formal neighbourhoods on $(\Sigma, P)$ the preferred isomorphism

$$
\Upsilon_{\tilde{\mathfrak{F}}}: \mathcal{V}_{\vec{\lambda}}^{\dagger}(\tilde{\mathfrak{F}}) \rightarrow \Psi_{\widetilde{\mathfrak{F}}}^{*} \mathcal{V}_{\lambda}^{\dagger}(\Sigma, P)
$$

given in Proposition 5.2 preserves connections.

This follows directly from Lemma 6.2.

Proposition 6.5. The $\mathbb{R}_{+}^{\mathrm{P}}$-action on $\mathcal{T}_{(\Sigma, P)}$ lifts by the use of the connection $\nabla^{(\vec{\alpha}, \vec{\beta})}$ to an action $\mathcal{V}_{\lambda}^{\dagger}$ of $\mathbb{R}_{+}^{\mathrm{P}}$ on $\mathcal{V}_{\lambda}^{\dagger}(\Sigma, P)$ which preserves the connection $\nabla^{(\vec{\alpha}, \vec{\beta})}$.

Proof. Since the $\mathbb{R}_{+}^{\mathrm{P}}$ action on the formal coordinates is just obtained by scaling the coordinates by positive scalars, we get a well defined homomorphism from $\mathbb{R}_{+}^{P}$ to the group of formal coordinates changes for any family of $N$-pointed Riemann surfaces with formal coordinates, hence by composing with the group homomorphism $G$ we get an action of $\mathbb{R}_{+}^{\mathrm{P}}$ on $\mathcal{V}_{\lambda}^{\dagger}(\Sigma, P)$. Note that we have here used Theorem 3.2.4. (2) for $p=0$ in [19], but only for these special real coordinates changes. This action preserved the connection by Theorem 3.2.4. (3) in [19].

Let now $\boldsymbol{\Sigma}_{i}, i=1,2$ be marked surface and let $f_{j}:\left(\Sigma_{1}, P_{1}\right) \rightarrow\left(\Sigma_{2}, P_{2}\right), j=1,2$ be diffeomorphisms of pointed surfaces, which induce the same morphism of marked surfaces from $\boldsymbol{\Sigma}_{1}$ to $\boldsymbol{\Sigma}_{2}$. Then there exists a unique $v \in \mathbb{R}_{+}^{\mathrm{P}}$ such that $v \cdot d_{P_{1}} f_{1}=d_{P_{2}} f_{2}$.

Lemma 6.3. We have a commutative diagram

$$
\begin{array}{ll}
\mathcal{V}_{\lambda_{1}}^{\dagger}\left(\Sigma_{1}, P_{1}\right) & \stackrel{\mathcal{V}^{\dagger}\left(f_{1}\right)}{\longrightarrow} \\
=\downarrow & \mathcal{V}_{\lambda_{2}}^{\dagger}\left(\Sigma_{2}, P_{2}\right) \\
\mathcal{V}_{\lambda_{1}}^{\dagger}\left(\Sigma_{1}, P_{1}\right) & \stackrel{\mathcal{V}^{\dagger}\left(f_{2}\right)}{\longrightarrow} \mathcal{V}_{\lambda_{2}}^{\dagger}\left(\Sigma_{2}, P_{2}\right),
\end{array}
$$


Proof. By the construction of $\mathcal{V}_{\lambda_{i}}^{\dagger}\left(\Sigma_{i}, P_{i}\right)$ we just need to check the commutativity on the $\mathcal{H}$-level, where this just amounts to $G$ being a homomorphism, which again is the content of Theorem 3.2.4. (2) in [19].

Let $(\Sigma, P, \lambda)$ be a general labeled pointed surface which might not be stable or saturated. Let now $(\tilde{P}, \tilde{\lambda})$ be obtained from $(P, \lambda)$ by labeling further points not in $P$ by $0 \in P_{\ell}$ such that $(\tilde{\Sigma}, \tilde{P}, \tilde{\lambda})$ is a stable and saturated labeled pointed surface. Let $\tilde{\pi}: \mathcal{T}_{(\Sigma, \tilde{P})} \rightarrow \mathcal{T}_{(\Sigma, P)}$ be the natural projection map.

Proposition 6.6. The connection $\nabla^{(\vec{\alpha}, \vec{\beta})}=\nabla^{(\vec{\alpha}, \vec{\beta})}(\Sigma, P)$ is flat with trivial holonomy when restricted to any of the fibers of $\tilde{\pi}: \mathcal{T}_{(\Sigma, \tilde{P})} \rightarrow \mathcal{T}_{(\Sigma, P)}$.

Proof. The propagation of vacua isomorphism applied along the fibers of $\tilde{\pi}$ is compatible with the connection. In the notation of chapter 5 of [19] this is seen by the following calculation. Let $\tilde{N}=|\tilde{P}|$. Let $\mathfrak{F}$ be a family of $\tilde{N}$-pointed curves with formal neighbourhoods on $(\Sigma, \tilde{P})$ such that $\Psi_{\mathfrak{F}}(\mathcal{B})$ is contained in a fiber of $\tilde{\pi}$. Further we can assume that when we consider $\mathfrak{F}$ over $(\Sigma, P)$, then it is simply a product family of a fixed $N$-pointed curve with formal neighbourhoods crossed with the base $\mathcal{B}$, i.e. we only vary the coordinates and the points in $\tilde{P}-P$. Then for any tangent field $X$ on $\mathcal{B}$, we choose a corresponding $\vec{\ell}=\left(\ell_{1}, \ldots, \ell_{N}, \ell_{N+1}, \ldots, \ell_{\tilde{N}}\right) \in \mathcal{L}(\mathfrak{F})$, such that $\ell_{j}=0, j=1, \ldots, N$ and $\ell_{j} \in \mathbb{C}[[\xi]], j=N+1, \ldots, \tilde{N}$. Then for a section of $\mathcal{H}_{\tilde{\lambda}}(\mathcal{B})$ of the form $F \otimes|\Phi\rangle \otimes|0\rangle \otimes \ldots \otimes|0\rangle$ we compute that

$$
\begin{aligned}
\nabla_{X}^{(\omega)}(F|\Phi\rangle \otimes|0\rangle \otimes \ldots \otimes|0\rangle)= & X(F)|\Phi\rangle \otimes|0\rangle \otimes \ldots \otimes|0\rangle \\
& -F \sum_{j=N+1}^{\tilde{N}} \rho_{j}\left(T\left[\ell_{j}\right]\right)(|\Phi\rangle \otimes|0\rangle \otimes \ldots \otimes|0\rangle) \\
& -a_{\omega}(\vec{\lambda}) F|\Phi\rangle \otimes|0\rangle \otimes \ldots \otimes|0\rangle \\
= & X(F)|\Phi\rangle) \otimes|0\rangle \otimes \ldots \otimes|0\rangle .
\end{aligned}
$$

Here we have used that $\rho_{j}\left(T\left[\ell_{j}\right]\right)(|0\rangle)=0$ because $\ell_{j} \in \mathbb{C}[[\xi]]$ and $a_{\omega}(\vec{\lambda})=0$ for the same reason. Hence we see that $\nabla^{(\omega)}$ is the trivial connection in $\mathcal{H}_{\tilde{\lambda}}(\mathcal{B})$. Hence the connection is flat with trivial holonomy on the subbundle $\mathcal{V}_{\tilde{\lambda}}^{\dagger}(\mathfrak{F}) \subset \mathcal{H}_{\tilde{\lambda}}^{\dagger}(\mathcal{B})$.

Let $(\Sigma, P, \lambda)$ be a general labeled pointed surface, i.e. $(\Sigma, P)$ might not be stable nor saturated. Let $\left(\Sigma, P^{\prime}, \lambda^{\prime}\right)$ and $\left(\Sigma, P^{\prime \prime}, \lambda^{\prime \prime}\right)$ be labeled marked surfaces obtained from $(\Sigma, P, \lambda)$ by labeling further points not in $P$ by $0 \in P_{\ell}$. Assume that $\left(\Sigma, P^{\prime}\right)$ and $\left(\Sigma, P^{\prime \prime}\right)$ are stable and saturated pointed surfaces. Let $\bar{P}=P^{\prime} \cup P^{\prime \prime}$ and $\bar{\lambda}$ be the induced labeling of $\bar{P}$. Note that $(\Sigma, \bar{P})$ is also stable and saturated. 
Proposition 6.7. Let $(\vec{\alpha}, \vec{\beta})$ be a symplectic basis of $H_{1}(\Sigma, \mathbb{Z})$. The isomorphisms given in Theorem 5.3 satisfies

$$
\nabla^{(\vec{\alpha}, \vec{\beta})}(\Sigma, \bar{P})=\left(\pi^{\prime}\right)^{*} \nabla^{(\vec{\alpha}, \vec{\beta})}\left(\Sigma, P^{\prime}\right)=\left(\pi^{\prime \prime}\right)^{*} \nabla^{(\vec{\alpha}, \vec{\beta})}\left(\Sigma, P^{\prime \prime}\right)
$$

Proof. We only have to consider the case of adding one point to a stable and saturated curve, i.e. say $\bar{P}=P^{\prime}$ and $P^{\prime}$ is obtained from $P^{\prime \prime}$ by adding one more point. Let $\bar{N}$ be the number of points in $\bar{P}$ and $\mathfrak{F}$ be a family of $\bar{N}$-pointed curves with formal neighbourhoods on $(\Sigma, \bar{P})$. For any tangent field $X$ on $\mathcal{B}$, we choose a corresponding $\vec{\ell}=\left(\ell_{1}, \ldots, \ell_{\bar{N}}, \ell_{\bar{N}+1}\right) \in \mathcal{L}(\mathfrak{F})$, such that $\ell_{\bar{N}+1} \in \mathbb{C}[[\xi]]$. Then the same computation as above shows that

$$
\nabla^{(\omega)}(F|\Phi\rangle \otimes|0\rangle)=\nabla^{(\omega)}(F|\Phi\rangle) \otimes|0\rangle
$$

The Proposition follows directly from this.

Let now $\left(\Sigma_{i}, P_{i}^{\prime}, \lambda_{i}^{\prime}\right)$ and $\left(\Sigma_{i}, P_{i}^{\prime \prime}, \lambda_{i}^{\prime \prime}\right)$ be stable and saturated labeled pointed surfaces, obtained from the labeled pointed surfaces $\left(\Sigma_{i}, P_{i}, \lambda_{i}\right)$, by labeling further points with the zero-label. Assume that $f^{\prime}:\left(\Sigma_{1}, P_{1}^{\prime}, \lambda_{1}^{\prime}\right) \rightarrow\left(\Sigma_{2}, P_{2}^{\prime}, \lambda_{2}^{\prime}\right)$ and $f^{\prime \prime}:\left(\Sigma_{1}, P_{1}^{\prime \prime}, \lambda_{1}^{\prime \prime}\right) \rightarrow\left(\Sigma_{2}, P_{2}^{\prime \prime}, \lambda_{2}^{\prime \prime}\right)$ are diffeomorphisms which induce isotopic maps from $\left(\Sigma_{1}, P_{1}\right)$ to $\left(\Sigma_{2}, P_{2}\right)$, where the isotopy is through maps which induces the same map from $P T_{P_{1}} \Sigma_{1}$ to $P T_{P_{2}} \Sigma_{2}$.

Proposition 6.8. With respect to the propagation of vacua isomorphisms, we get that

$$
\left(\pi^{\prime}\right)^{*} \mathcal{V}^{\dagger}\left(f^{\prime}\right)=\left(\pi^{\prime \prime}\right)^{*} \mathcal{V}^{\dagger}\left(f^{\prime \prime}\right)
$$

This follows directly from the way the morphisms for $f^{\prime}$ and $f^{\prime \prime}$ are defined.

7. Definition of the space of abelian vacua associated to a Riemann surface.

7.1. Fermion Fock space. Let $\mathbf{Z}_{h}$ be the set of all half integers. Namely

$$
\mathbf{Z}_{h}=\{n+1 / 2 \mid n \in \mathbf{Z}\}
$$

Let $\mathcal{W}^{\dagger}$ be an infinite-dimensional vector space over $\mathbf{C}$ with a filtration $\left\{F^{m} \mathcal{W}^{\dagger}\right\}_{m \in \mathbf{Z}}$ which satisfies the following conditions.

(1) The filtration $\left\{F^{m} \mathcal{W}^{\dagger}\right\}$ is decreasing;

(2) $\bigcup_{m \in \mathbf{Z}} F^{m} \mathcal{W}^{\dagger}=\mathcal{W}^{\dagger}, \quad \bigcap_{m \in \mathbf{Z}} F^{m} \mathcal{W}^{\dagger}=\{0\}$

(3) $\operatorname{dim}_{\mathbf{C}} F^{m} \mathcal{W}^{\dagger} / F^{m+1} \mathcal{W}^{\dagger}=1$;

(4) The vector space $\mathcal{W}^{\dagger}$ is complete with respect to the uniform topology such that $\left\{F^{m} \mathcal{W}^{\dagger}\right\}$ is a basis of open neighbourhoods of 0 . 
We introduce a basis $\left\{e^{\nu}\right\}_{\nu \in \mathbf{Z}_{h}}$ of $\mathcal{W}^{\dagger}$ in such a way that

$$
e^{m+1 / 2} \in F^{m} \mathcal{W}^{\dagger} \backslash F^{m+1} \mathcal{W}^{\dagger}
$$

Then, each element $u \in \mathcal{W}^{\dagger}$ can uniquely be expressed in the form

$$
u=\sum_{\nu>n_{0}, \nu \in \mathbf{Z}_{h}}^{\infty} a_{\nu} e^{\nu}
$$

for some $n_{0}$ and with respect to this basis the filtration is given by

$$
F^{m} \mathcal{W}^{\dagger}=\left\{u \in \mathcal{W}^{\dagger} \mid u=\sum_{\nu>m, \nu \in \mathbf{Z}_{h}}^{\infty} a_{\nu} e^{\nu}\right\} \text {. }
$$

We fix the basis $\left\{e^{\nu}\right\}_{\nu \in \mathbf{Z}_{h}}$ throughout the present paper.

Let $\mathbf{C}((\xi))$ be a field of formal Laurent series over the complex number field. Then the basis gives us a filtration preserving linear isomorphism

$$
\begin{aligned}
\mathbf{C}((\xi)) & \cong \mathcal{W}^{\dagger} \\
\xi^{n} & \mapsto e^{n+1 / 2} .
\end{aligned}
$$

By mapping $\xi^{n} d \xi$ to $e^{n+1 / 2}$ we of course also get a filtration preserving linear isomorphism between $\mathbf{C}((\xi)) d \xi$ and $\mathcal{W}^{\dagger}$.

We let $\left\{\bar{e}_{\nu}\right\}_{\nu \in \mathbf{Z}_{h}}$ be the dual basis of $\left\{e^{\nu}\right\}_{\nu \in \mathbf{Z}_{h}}$. Then, put

$$
\mathcal{W}=\bigoplus_{\nu \in \mathbf{Z}_{h}} \mathbf{C} \bar{e}_{\nu}
$$

Then $\mathcal{W}$ is the topological dual of the vector space $\mathcal{W}^{\dagger}$. There is a natural pairing $(\quad \mid): \mathcal{W}^{\dagger} \times \mathcal{W} \rightarrow$ C defined by

$$
\left(e^{\nu} \mid \bar{e}_{\mu}\right)=\delta_{\mu}^{\nu}
$$

In other word we have

$$
(u \mid v)=v(u) .
$$

Let us introduce the semi-infinite exterior product of the vector spaces $\mathcal{W}$ and $\mathcal{W}^{\dagger}$. For that purpose we first introduce the notion of a Maya diagram.

Definition 7.1. A Maya diagram $M$ of the charge $p, p \in \mathbf{Z}$ is a set

$$
M=\{\mu(p-1 / 2), \mu(p-3 / 2), \mu(p-5 / 2), \ldots\},
$$

where $\mu$ is an increasing function

$$
\mu: \mathbf{Z}_{h<p}=\left\{\nu \in \mathbf{Z}_{h} \mid \nu<p\right\} \rightarrow \mathbf{Z}_{h}
$$

such that there exists an integer $n_{0}$ such that

$$
\mu(\nu)=\nu
$$


for all $\nu<n_{0}$.

The function $\mu$ is called the characteristic function of the Maya diagram $M$. The set of Maya diagrams of charge $p$ is written as $\mathcal{M}_{p}$.

For a Maya diagram $\mathrm{M}$ we have $\mu(\nu)=\nu$ for almost all $\nu$. Therefore the set

$$
\left\{\mu(\nu)-\nu \mid \nu \in \mathbf{Z}_{h}, \mu(\nu)-\nu>0\right\}
$$

is finite and the number

$$
d(M)=\sum_{\nu \in \mathbf{Z}_{h}}(\mu(\nu)-\nu)
$$

is finite. The number $d(M)$ is also written as $d(\mu)$ and it is called the degree of the Maya diagram $M$ with characteristic function $\mu$. The finite set of Maya diagrams of degree $d$ and change $p$ is denoted $\mathcal{M}_{p}^{d}$. Clearly $\mathcal{M}_{p}=\coprod_{d} \mathcal{M}_{p}^{d}$.

For a Maya diagram $M$ of charge $p$ we define two semi-infinite products

$$
\begin{aligned}
|M\rangle & =\bar{e}_{\mu(p-1 / 2)} \wedge \bar{e}_{\mu(p-3 / 2)} \wedge \bar{e}_{\mu(p-5 / 2)} \wedge \cdots \\
\langle M| & =\cdots \wedge e^{\mu(p-5 / 2)} \wedge e^{\mu(p-3 / 2)} \wedge e^{\mu(p-1 / 2)}
\end{aligned}
$$

Formally, these semi-infinite products is just another notation for the corresponding Maya diagram. This notation is particular convenient for the following discussion. However, by using the basis $e_{\nu}$, we clear indicate the relation to the vector spaces $\mathcal{W}$ and $\mathcal{W}^{\dagger}$.

For any integer $p$ put

$$
\begin{aligned}
|p\rangle & =\bar{e}_{p-1 / 2} \wedge \bar{e}_{p-3 / 2} \wedge \bar{e}_{p-5 / 2} \wedge \cdots \\
\langle p| & =\cdots \wedge e^{p-5 / 2} \wedge e^{p-3 / 2} \wedge e^{p-1 / 2}
\end{aligned}
$$

Now the fermion Fock space $\mathcal{F}^{\dagger}(p)$ of charge $p$ and the dual fermion Fock space $\mathcal{F}(p)$ of charge $p$ are defined by

$$
\begin{aligned}
\mathcal{F}(p) & =\bigoplus_{M \in \mathcal{M}_{p}} \mathbf{C}|M\rangle \\
\mathcal{F}^{\dagger}(p) & =\prod_{M \in \mathcal{M}_{p}} \mathbf{C}\langle M|
\end{aligned}
$$

We observe that

$$
\mathcal{F}(p)=\bigoplus_{d \geq 0} \mathcal{F}_{d}(p)
$$

where

$$
\mathcal{F}_{d}(p)=\bigoplus_{M \in \mathcal{M}_{p}^{d}} \mathbf{C}|M\rangle
$$

The dual pairing

$$
\langle\cdot \mid \cdot\rangle: \mathcal{F}^{\dagger}(p) \times \mathcal{F}(p) \rightarrow \mathbf{C}
$$


is given by

$$
\langle M \mid N\rangle=\delta_{M, N}, \quad M, N \in \mathcal{M}_{p}
$$

Put also

$$
\begin{aligned}
\mathcal{F} & =\bigoplus_{p \in \mathbf{Z}} \mathcal{F}(p) \\
\mathcal{F}^{\dagger} & =\bigoplus_{p \in \mathbf{Z}} \mathcal{F}^{\dagger}(p)
\end{aligned}
$$

The vector space $\mathcal{F}^{\dagger}$ is called the fermion Fock space and $\mathcal{F}$ is called the dual fermion Fock space. These are the semi-infinite exterior products of the vector spaces $\mathcal{W}^{\dagger}$ and $\mathcal{W}$ respectively, which we shall be interested in. We only define the fermion Fock space by using the basis $e_{\nu}$, since we are fixing this basis throughout.

The above pairing can be extended to the one on $\mathcal{F}^{\dagger} \times \mathcal{F}$ by assuming that the paring is zero on $\mathcal{F}^{\dagger}(p) \times \mathcal{F}\left(p^{\prime}\right)$ if $p \neq p^{\prime}$.

Let us introduce the fermion operators $\psi_{\nu}$ and $\bar{\psi}_{\nu}$ for all half integers $\nu \in \mathbf{Z}_{h}$ which act on $\mathcal{F}$ from the left and on $\mathcal{F}^{\dagger}$ from the right.

$$
\begin{aligned}
\text { Left action on } \mathcal{F} & \psi_{\nu}=i\left(\bar{e}_{\nu}\right), \quad \bar{\psi}_{\nu}=\bar{e}_{-\nu} \wedge \\
\text { Right action on } \mathcal{F}^{\dagger} & \psi_{\nu}=\wedge e^{\nu}, \quad \bar{\psi}_{\nu}=i\left(e^{-\nu}\right)
\end{aligned}
$$

where $i(\cdot)$ is the interior product. For example we have

$$
\begin{aligned}
\psi_{-3 / 2}|0\rangle & =i\left(\bar{e}_{-3 / 2}\right) \bar{e}_{-1 / 2} \wedge \bar{e}_{-3 / 2} \wedge \cdots=-\bar{e}_{-1 / 2} \wedge \bar{e}_{-5 / 2} \wedge \bar{e}_{-7 / 2} \wedge \cdots \\
\langle 0| \bar{\psi}_{5 / 2} & =\cdots \wedge e^{-5 / 2} \wedge e^{-3 / 2} \wedge e^{-1 / 2} i\left(e^{-5 / 2}\right)=\cdots \wedge e^{-7 / 2} \wedge e^{-3 / 2} \wedge e^{-1 / 2}
\end{aligned}
$$

Note that $\psi_{\nu}$ maps $\mathcal{F}(p)$ to $\mathcal{F}(p-1)$, hence decreases the charge by one, and $\bar{\psi}_{\nu}$ maps $\mathcal{F}(p)$ to $\mathcal{F}(p+1)$, hence increase the charge by one. Similarly the right action of $\psi_{\nu}$ maps $\mathcal{F}^{\dagger}(p)$ to $\mathcal{F}^{\dagger}(p+1)$ and $\bar{\psi}_{\nu}$ maps $\mathcal{F}^{\dagger}(p)$ to $\mathcal{F}^{\dagger}(p-1)$. It is easy to show that for any $\langle u| \in \mathcal{F}$ and $|v\rangle \in \mathcal{F}^{\dagger}$ we have

$$
\left\langle u \mid \psi_{\nu} v\right\rangle=\left\langle u \psi_{\nu} \mid v\right\rangle, \quad\left\langle u \mid \bar{\psi}_{\nu} v\right\rangle=\left\langle u \bar{\psi}_{\nu} \mid v\right\rangle .
$$

The fermion operators have the following anti-commutation relations as operators on $\mathcal{F}$ and $\mathcal{F}^{\dagger}$.

$$
\begin{aligned}
& {\left[\psi_{\nu}, \psi_{\mu}\right]_{+}=0,} \\
& {\left[\bar{\psi}_{\nu}, \bar{\psi}_{\mu}\right]_{+}=0,} \\
& {\left[\psi_{\nu}, \bar{\psi}_{\mu}\right]_{+}=\delta_{\nu+\mu, 0},}
\end{aligned}
$$

where we define

$$
[A, B]_{+}=A B+B A .
$$

Note that for each Maya diagram $M$ of charge $p$ we can find non-negative half integers

$$
\mu_{1}<\mu_{2}<\cdots<\mu_{r}<0, \quad \nu_{1}<\nu_{2}<\cdots<\nu_{s}<0, \quad r \geq 0, s \geq 0
$$


with $r-s=p$ and $\mu_{i} \neq \nu_{j}$ such that

$$
|M\rangle=(-1)^{\sum_{i=1}^{s} \nu_{i}+s / 2} \bar{\psi}_{\mu_{1}} \bar{\psi}_{\mu_{2}} \cdots \bar{\psi}_{\mu_{r}} \psi_{\nu_{s}} \psi_{\nu_{s-1}} \cdots \psi_{\nu_{1}}|0\rangle .
$$

The negative half integers $\mu_{i}$ 's and $\nu_{j}$ 's are uniquely determined by the Maya diagram $M$.

The normal ordering: $:$ of the fermion operators are defined as follows.

$$
A_{\nu} B_{\mu}:= \begin{cases}-B_{\mu} A_{\nu} & \text { if } \mu<0 \text { and } \nu>0 \\ A_{\nu} B_{\mu} & \text { otherwise }\end{cases}
$$

where $A$ and $B$ is $\psi$ or $\bar{\psi}$. By (49), (50) and (51) the normal ordering is non-trivial if and only if $\mu<0$ and $A_{\nu}=\bar{B}_{-\mu}$.

The field operators $\psi(z)$ and $\bar{\psi}(z)$ are defined by

$$
\begin{aligned}
\psi(z) & =\sum_{\mu} \psi_{\mu} z^{-\mu-1 / 2}, \\
\bar{\psi}(z) & =\sum_{\mu} \bar{\psi}_{\mu} z^{-\mu-1 / 2} .
\end{aligned}
$$

The current operator $J(z)$ is defined by

$$
J(z)=: \bar{\psi}(z) \psi(z):=\sum_{n \in \mathbf{Z}} J_{n} z^{-n-1}
$$

Note that thanks to the normal ordering, the operator $J_{n}$ can operate on $\mathcal{F}$ and $\mathcal{F}^{\dagger}$ even though $J_{n}$ is an infinite sum of operators.

The energy-momentum tensor $T(z)$ is defined by

$$
T(z)=: \frac{d \psi(z)}{d z} \bar{\psi}(z):=\sum_{n \in \mathbf{Z}} L_{n} z^{-n-2} .
$$

Again due to the normal ordering, the coefficients $L_{n}$ operates on $\mathcal{F}$ and $\mathcal{F}^{\dagger}$. The set $\left\{L_{n}\right\}_{n \in \mathbf{Z}}$ forms the Virasoro algebra with central charge $c=-2$.

The field operators $\psi(z)$ and $\bar{\psi}(z)$, the current operator $J(z)$ and the energy-momentum tensor $T(z)$ form the so-called spin $j=0 b c$-system or ghost system in the physics literature.

7.2. Abelian Vacua. For a positive integer $N$ put

$$
\begin{aligned}
\mathcal{F}_{N} & =\bigoplus_{p_{1}, \ldots, p_{N} \in \mathbf{Z}} \mathcal{F}\left(p_{1}\right) \otimes \cdots \otimes \mathcal{F}\left(p_{N}\right), \\
\mathcal{F}_{N}^{\dagger} & =\bigoplus_{p_{1}, \ldots, p_{N} \in \mathbf{Z}} \mathcal{F}^{\dagger}\left(p_{1}\right) \hat{\otimes} \cdots \hat{\otimes} \mathcal{F}^{\dagger}\left(p_{N}\right),
\end{aligned}
$$

where $\hat{\otimes}$ means the complete tensor product. 
Definition 7.2. Let $\mathfrak{X}=\left(C ; q_{1}, q_{2}, \ldots, q_{N} ; \xi_{1}, \xi_{2}, \ldots, \xi_{N}\right)$ be a saturated pointed Riemann Surface with formal neighbourhoods. The abelian vacua (ghost vacua in [2]) $\mathcal{V}_{\mathrm{ab}}^{\dagger}(\mathfrak{X})$ of the spin $j=0$ ghost system associated to $\mathfrak{X}$ is a linear subspace of $\mathcal{F}_{N}^{\dagger}$ consisting of elements $\langle\Phi|$ satisfying the following conditions:

(1) For all $|v\rangle \in \mathcal{F}_{N}$, there exists a meromorphic function $f \in H^{0}\left(C, \mathcal{O}_{C}\left(* \sum_{j=1}^{N} q_{j}\right)\right)$ such that $\left\langle\Phi\left|\rho_{j}\left(\psi\left(\xi_{j}\right)\right)\right| v\right\rangle$ is the Laurent expansion of $f$ at the point $q_{j}$ with respect to the formal coordinate $\xi_{j}$;

(2) For all $|v\rangle \in \mathcal{F}_{N}$, there exists a meromorphic one-form $\omega \in H^{0}\left(C, \omega_{C}\left(* \sum_{j=1}^{N} q_{j}\right)\right)$ such that $\left\langle\Phi\left|\rho_{j}\left(\bar{\psi}\left(\xi_{j}\right)\right)\right| v\right\rangle d \xi_{j}$ is the Laurent expansion of $\omega$ at the point $q_{j}$ with respect to the coordinates $\xi_{j}$,

where $\rho_{j}(A)$ means that the operator $A$ acts on the $j$-th component of $\mathcal{F}_{N}$ as

$$
\rho_{j}(A)\left|u_{1} \otimes u_{2} \otimes \cdots \otimes u_{N}\right\rangle=(-1)^{p_{1}+\cdots+p_{j-1}}\left|u_{1} \otimes \cdots \otimes u_{j-1} \otimes A u_{j} \otimes u_{j+1} \otimes \cdots \otimes u_{N}\right\rangle .
$$

We will reformulate the above two conditions into gauge conditions. For that purpose we introduce the following notation.

For a meromorphic one-form $\omega \in H^{0}\left(C, \omega_{C}\left(* \sum_{j=1}^{N} q_{j} j\right)\right)$ we let

$$
\omega_{j}=\left(\sum_{n=-n_{0}}^{\infty} a_{n} \xi_{j}^{n}\right) d \xi_{j}
$$

be the Laurent expansion at $q_{j}$ with respect to the coordinate $\xi_{j}$. Then, for the field operator $\psi(z)$ let us define $\psi\left[\omega_{j}\right]$ by

$$
\psi\left[\omega_{j}\right]=\operatorname{Res}_{\xi_{j}=0}\left(\psi\left(\xi_{j}\right) \omega_{j}\right)=\sum_{n=-n_{0}}^{\infty} a_{n} \psi_{n+1 / 2} .
$$

Similarly we can define $\bar{\psi}\left[\omega_{j}\right]$. For a meromorphic function $f \in H^{0}\left(C, \mathcal{O}_{C}\left(* \sum_{j=1}^{N} q_{j}\right)\right)$ we let $f_{j}\left(\xi_{j}\right)$ be the Laurent expansion of $f$ at $q_{j}$ with respect to the coordinate $\xi_{j}$. For the field operator $\psi(z)$ define $\psi\left[f_{j}\right]$ by

$$
\psi\left[f_{j}\right]=\operatorname{Res}_{\xi_{j}=0}\left(\psi\left(\xi_{j}\right) f_{j}\left(\xi_{j}\right) d \xi_{j}\right)
$$

Put

$$
\begin{aligned}
\psi[\omega] & =\left(\psi\left[\omega_{1}\right], \ldots, \psi\left[\omega_{N}\right]\right), & & \bar{\psi}[\omega]=\left(\bar{\psi}\left[\omega_{1}\right], \ldots, \bar{\psi}\left[\omega_{N}\right]\right) \\
\psi[f] & =\left(\psi\left[f_{1}\right], \ldots, \psi\left[f_{N}\right]\right), & \bar{\psi}[f] & =\left(\bar{\psi}\left[f_{1}\right], \ldots, \bar{\psi}\left[f_{N}\right]\right) .
\end{aligned}
$$


Then, these operate on $\mathcal{F}_{N}$ from the left and on $\mathcal{F}_{N}^{\dagger}$ from the right. For example, $\bar{\psi}[f]$ operates on $\mathcal{F}_{N}$ from the left by

$$
\begin{aligned}
\bar{\psi}[f]\left|u_{1} \otimes \cdots \otimes u_{N}\right\rangle & =\sum_{j=1}^{N} \rho_{j}\left(\bar{\psi}\left[f_{j}\right]\right)\left|u_{1} \otimes \cdots \otimes u_{N}\right\rangle \\
& =\sum_{j=1}^{N}(-1)^{p_{1}+\cdots+p_{j-1}}\left|u_{1} \otimes \cdots \otimes u_{j-1} \otimes \bar{\psi}\left[f_{j}\right] u_{j} \otimes u_{j+1} \otimes \cdots \otimes u_{N}\right\rangle
\end{aligned}
$$

for $\left|u_{j}\right\rangle \in \mathcal{F}^{\dagger}\left(p_{j}\right)$ and operates on $\mathcal{F}_{N}^{\dagger}$ from the right by

$$
\begin{aligned}
\left\langle v_{N} \otimes \cdots v_{1}\right| \bar{\psi}[f] & =\sum_{j=1}^{N}\left\langle v_{N} \otimes \cdots v_{1}\right| \rho_{j}\left(\bar{\psi}\left[f_{j}\right]\right) \\
& =\sum_{j=1}^{N}(-1)^{p_{1}+\cdots+p_{j-1}}\left\langle v_{N} \otimes \cdots \otimes v_{j+1} \otimes v_{j} \bar{\psi}\left[f_{j}\right] \otimes v_{j-1} \otimes \cdots \otimes v_{1}\right|
\end{aligned}
$$

for $\left\langle v_{j}\right| \in \mathcal{F}^{\dagger}\left(p_{j}\right)$.

Theorem 7.1 ([2, Theorem 3.1]). The element $\langle\Phi| \in \mathcal{F}_{N}^{\dagger}$ belongs to the space of abelian vacua $\mathcal{V}_{\mathrm{ab}}^{\dagger}(\mathfrak{X})$ of the $j=0$ ghost system if and only if $\langle\Phi|$ satisfies the following two conditions.

(1) $\langle\Phi| \psi[\omega]=0$ for any meromorphic one-form $\omega \in H^{0}\left(C, \omega_{C}\left(* \sum_{j=1}^{N} q_{j}\right)\right)$.

(2) $\langle\Phi| \bar{\psi}[f]=0$ for any meromorphic function $f \in H^{0}\left(C, \mathcal{O}_{C}\left(* \sum_{j=1}^{N} q_{j}\right)\right)$.

The first (resp. second) condition in the above theorem is called the first (resp. second) gauge condition. The first and second gauge conditions can be rewritten in the following form:

(1) $\sum_{j=1}^{N}(-1)^{p_{1}+\cdots+p_{j-1}}\left\langle\Phi \mid u_{1} \otimes \cdots \otimes \cdots \otimes u_{j-1} \otimes \psi\left[\omega_{j}\right] u_{j} \otimes u_{j+1} \otimes \cdots \otimes u_{N}\right\rangle=0$ for any $\omega \in H^{0}\left(C, \omega_{C}\left(* \sum_{j=1}^{N} q_{j}\right)\right)$ and $\left|u_{j}\right\rangle \in \mathcal{F}\left(p_{j}\right), j=1,2, \ldots, N$.

(2) $\sum_{j=1}^{N}(-1)^{p_{1}+\cdots+p_{j-1}}\left\langle\Phi \mid u_{1} \otimes \cdots \otimes u_{j-1} \otimes \bar{\psi}\left[f_{j}\right] u_{j} \otimes u_{j+1} \otimes \cdots \otimes u_{N}\right\rangle=0$ for any $f \in$ $H^{0}\left(C, \mathcal{O}_{C}\left(* \sum_{j=1}^{N} q_{j}\right)\right)$ and $\left|u_{j}\right\rangle \in \mathcal{F}\left(p_{j}\right), j=1,2, \ldots, N$.

It is easy to show that the abelian vacua $\mathcal{V}_{\mathrm{ab}}^{\dagger}(\mathfrak{X})$ is a finite dimensional vector space. More strongly we can prove the following theorem.

Theorem $7.2([2$, Theorem 3.2$])$. For any pointed Riemann surface $\mathfrak{X}=\left(C ; Q_{1}, \ldots, Q_{N} ; \xi_{1}, \ldots \xi_{N}\right)$ with formal neighbourhoods we have

$$
\operatorname{dim}_{\mathbf{C}} \mathcal{V}_{\mathrm{ab}}^{\dagger}(\mathfrak{X})=1
$$

In the later application we need to consider a disconnected Riemann surface. The following proposition is an immediate consequence of the definition.

Proposition 7.1 ([2, Proposition 3.1]). Let

$$
\mathfrak{X}_{1}=\left(C_{1} ; q_{1}, \ldots, q_{M} ; \xi_{1}, \ldots, \xi_{M}\right)
$$


and

$$
\mathfrak{X}_{2}=\left(C_{2} ; q_{M+1}, \ldots, q_{M} ; \xi_{M+1}, \ldots, \xi_{N}\right)
$$

be saturated pointed Riemann surfaces with formal neighbourhoods. Let $C$ be the disjoint union $C_{1} \sqcup C_{2}$ of the Riemann surfaces $C_{1}, C_{2}$. Put

$$
\mathfrak{X}=\left(C ; q_{1}, \ldots, q_{N} ; \xi_{1}, \ldots, \xi_{N}\right) .
$$

Then we have

$$
\mathcal{V}_{\mathrm{ab}}^{\dagger}(\mathfrak{X})=\mathcal{V}_{\mathrm{ab}}^{\dagger}\left(\mathfrak{X}_{1}\right) \otimes \mathcal{V}_{\mathrm{ab}}^{\dagger}\left(\mathfrak{X}_{2}\right)
$$

Now we can introduce the dual abelian vacua.

Definition 7.3. Let $\mathcal{F}_{\mathrm{ab}}(\mathfrak{X})$ be the subspace of $\mathcal{F}_{N}$ spanned by $\psi[\omega] \mathcal{F}_{N}, \omega \in H^{0}\left(C, \omega_{C}\left(* \sum_{j=1}^{N} q_{j}\right)\right)$ and $\bar{\psi}[f] \mathcal{F}_{N}, f \in H^{0}\left(C, \mathcal{O}\left(* \sum_{j=1}^{N} q_{j}\right)\right)$. Put

$$
\mathcal{V}_{\mathrm{ab}}(\mathfrak{X})=\mathcal{F}_{N} / \mathcal{F}_{\mathrm{ab}}(\mathfrak{X}) .
$$

The quotients space $\mathcal{V}_{\mathrm{ab}}(\mathfrak{X})$ is called the space of dual abelian vacua or dual ghost vacua of the $j=0$ ghost system.

Since $\mathcal{V}_{\mathrm{ab}}^{\dagger}(\mathfrak{X})$ is finite dimensional, $\mathcal{V}_{\mathrm{ab}}(\mathfrak{X})$ is dual to $\mathcal{V}_{\mathrm{ab}}^{\dagger}(\mathfrak{X})$.

Theorem 7.3 ([2, Theorem 3.2.]). The space of abelian vacua $\mathcal{V}_{\mathrm{ab}}^{\dagger}(\mathfrak{X})$ is isomorphic to the determinant of the canonical bundle $\omega_{C}$

$$
\mathcal{V}_{\mathrm{ab}}^{\dagger}(\mathfrak{X}) \cong \operatorname{det}\left(H^{0}\left(C, \omega_{C}\right)\right) .
$$

Let $\mathfrak{X}=\left(C ; q_{1}, \ldots, q_{N} ; \xi_{1}, \ldots, \xi_{N}\right)$ be an $N$-pointed Riemann surface with formal neighbourhoods. Let $q_{N+1}$ be a non-singular point and choose a formal coordinate $\xi_{N+1}$ of $C$ with center $q_{N+1}$. Put

$$
\widetilde{\mathfrak{X}}=\left(C ; q_{1}, \ldots, q_{N}, q_{N+1} ; \xi_{1}, \ldots, \xi_{N}, \xi_{N+1}\right) .
$$

Then the canonical linear mapping

$$
\begin{aligned}
\iota: \mathcal{F}_{N} & \rightarrow \mathcal{F}_{N+1} \\
|v\rangle & \mapsto|v\rangle \otimes|0\rangle
\end{aligned}
$$

induces the canonical mapping

$$
\iota^{*}: \mathcal{F}_{N+1}^{\dagger} \rightarrow \mathcal{F}_{N}^{\dagger}
$$

Theorem 7.4 ([2, Theorem 3.4]). The canonical mapping $\iota^{*}$ induces an isomorphism

$$
\mathcal{V}_{\mathrm{ab}}^{\dagger}(\widetilde{\mathfrak{X}}) \cong \mathcal{V}_{\mathrm{ab}}^{\dagger}(\mathfrak{X})
$$


This isomorphism is denoted the "Propagation of abelian vacua" isomorphism.

Let us consider change of formal neighbourhoods. We use the same notation as in $\S 4.5$. For any automorphism $h \in \mathcal{D}_{+}^{0}$ we can define the action $G[h]$ on the fermion Fock space $\mathcal{F}$ by using the energy-momentum tensor (56) as in $\S 4.5$.

Proposition 7.2 ([2, Proposition 6.1]). For any $h_{j} \in \mathcal{D}_{+}^{0}, j=1,2, \ldots, N$ and $N$-pointed curve

$$
\mathfrak{X}=\left(C ; Q_{1}, Q_{2}, \ldots, Q_{n} ; \xi_{1}, \xi_{2}, \ldots, \xi_{N}\right)
$$

with formal neighbourhoods, put

$$
\mathfrak{X}_{(h)}=\left(C ; Q_{1}, Q_{2}, \ldots, Q_{N} ; h_{1}\left(\xi_{1}\right), h_{2}\left(\xi_{2}\right), \ldots, h_{N}\left(\xi_{N}\right)\right) .
$$

Then, the isomorphism $G\left[h_{1}\right] \widehat{\otimes} \cdots \widehat{\otimes} G\left[h_{N}\right]$

$$
\begin{aligned}
\mathcal{F}_{N}^{\dagger} & \rightarrow \mathcal{F}_{N}^{\dagger} \\
\left\langle\phi_{1} \widehat{\otimes} \cdots \widehat{\otimes} \phi_{N}\right| & \mapsto\left\langle\phi_{1} G\left[h_{1}\right] \widehat{\otimes} \cdots \widehat{\otimes} \phi_{N} G\left[h_{N}\right]\right|
\end{aligned}
$$

induces the canonical isomorphism

$$
G\left[h_{1}\right] \widehat{\otimes} \cdots \widehat{\otimes} G\left[h_{N}\right]: \mathcal{V}_{\mathrm{ab}}^{\dagger}(\mathfrak{X}) \rightarrow \mathcal{V}_{\mathrm{ab}}^{\dagger}\left(\mathfrak{X}_{(h)}\right)
$$

7.3. The space of abelian vacua associated to a Riemann surface. Let $\mathbf{C}$ be a compact Riemann surface. For a stable and saturated pointed Riemann surface with formal neighbourhoods $\mathfrak{X}$ we denote by $\tilde{c}(\mathfrak{X})$ the underlying Riemann surface. Suppose we now have two pointed Riemann surfaces with formal neighbourhoods $\mathfrak{X}_{i}$ such that $\tilde{c}\left(\mathfrak{X}_{1}\right)=\tilde{\mathrm{c}}\left(\mathfrak{X}_{2}\right)=\mathbf{C}$. Choose for each component of $\mathbf{C}$ a point with a formal neighbourhood, which is not a point with formal neighbourhoods of $\mathfrak{X}_{i}, \mathrm{i}=1,2$. Let $\mathfrak{X}_{0}$ be the resulting stable and saturated pointed Riemann surface with formal neighbourhoods (if $\mathfrak{X}_{0}$ is not stable, then add further points with formal neighbourhoods). Then iterations of the propagation of vacua isomorphism determined by the inclusion of $\mathcal{F}_{N}$ into $\mathcal{F}_{N+1}$ given by $|v\rangle \mapsto|v\rangle \otimes|0\rangle$, induces isomorphisms from $\mathcal{V}_{\mathrm{ab}}^{\dagger}\left(\mathfrak{X}_{0}\right)$ to $\mathcal{V}_{\mathrm{ab}}^{\dagger}\left(\mathfrak{X}_{i}\right), i=1,2$. It is elementary to check that the resulting isomorphism from $\mathcal{V}_{\mathrm{ab}}^{\dagger}\left(\mathfrak{X}_{1}\right)$ to $\mathcal{V}_{\mathrm{ab}}^{\dagger}\left(\mathfrak{X}_{2}\right)$ is independent of $\mathfrak{X}_{0}$.

Furthermore, we get from the commutativity of the following diagram

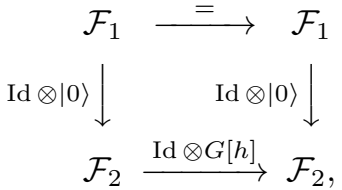

which follows from the fact that $G[h]|0\rangle=|0\rangle$, that these isomorphisms are also compatible with the change of formal coordinates isomorphism induced by $G[h]$.

Definition 7.4. The space of abelian vacua associated to the Riemann surface $\mathbf{C}$ is by definition

$$
\mathcal{V}_{\mathrm{ab}}^{\dagger}(\mathbf{C})=\coprod_{\tilde{\mathrm{c}}(\mathfrak{X})=\mathbf{C}} \mathcal{V}_{\mathrm{ab}}^{\dagger}(\mathfrak{X}) / \sim,
$$


where the disjoint union is over all Riemann surfaces with formal neighbourhoods with $\mathbf{C}$ as the underlying Riemann surface and $\sim$ is the equivalence relation generated by the isomorphisms discussed above.

It is obvious that

Proposition 7.3. The natural quotient map from $\mathcal{V}_{\mathrm{ab}}^{\dagger}(\mathfrak{X})$ to $\mathcal{V}_{\mathrm{ab}}^{\dagger}(\mathbf{C})$ is an isomorphism for all Riemann surfaces with formal neighbourhoods $\mathfrak{X}$ with $\tilde{\mathrm{c}}(\mathfrak{X})=\mathbf{C}$.

Suppose $\mathbf{C}_{i}, i=1,2$ are Riemann surfaces and $\Phi: \mathbf{C}_{1} \rightarrow \mathbf{C}_{2}$ is a morphism of labeled marked Riemann surfaces. Let $\mathfrak{X}_{2}$ be a Riemann surface with formal neighbourhoods such that $\tilde{\mathbf{c}}\left(\mathfrak{X}_{2}\right)=\mathbf{C}_{2}$. Let $\Phi^{*} \mathfrak{X}_{2}=\mathfrak{X}_{1}$. Then $\Phi$ is a morphism of Riemann surfaces with formal neighbourhoods. We clearly have that

Proposition 7.4. The identity map on $\mathcal{F}_{N}$ induces a linear isomorphism from $\mathcal{V}_{\mathrm{ab}}^{\dagger}\left(\mathfrak{X}_{1}\right)$ to $\mathcal{V}_{\mathrm{ab}}^{\dagger}\left(\mathfrak{X}_{2}\right)$, which induces a well defined linear isomorphism $\mathcal{V}_{\mathrm{ab}}^{\dagger}(\Phi)$ from $\mathcal{V}_{\mathrm{ab}}^{\dagger}\left(\mathbf{C}_{1}\right)$ to $\mathcal{V}_{\mathrm{ab}}^{\dagger}\left(\mathbf{C}_{2}\right)$. Compositions of morphisms of labeled marked Riemann surfaces go to compositions of the induced linear isomorphisms.

\section{Definition of the line Bundle of abelian vacua over Teichmüller space}

\subsection{Sheaf of abelian vacua. Let}

$$
\mathfrak{F}=\left(\pi: \mathcal{C} \rightarrow \mathcal{B} ; s_{1}, \ldots, s_{N} ; \xi_{1}, \ldots, \xi_{N}\right)
$$

be a family of $N$-pointed semi-stable curves with formal neighbourhoods. That is $\mathcal{C}$ and $\mathcal{B}$ are complex manifolds, $\pi$ is a proper holomorphic mapping, and for each point $b \in \mathcal{B}, \mathfrak{F}(b)=\left(C_{b}=\right.$ $\left.\pi^{-1}(b) ; s_{1}(b), \ldots, s_{N}(b) ; \xi_{1}, \ldots, \xi_{N}\right)$ is an $N$-pointed semi-stable curve with formal neighbourhoods. We let $\Sigma$ be the locus of double points of the fibers of $\mathfrak{F}$ and let $D$ be $\pi(\Sigma)$. Note that $\Sigma$ is a nonsingular submanifold of codimension two in $\mathcal{C}$, and $D$ is a divisor in $\mathcal{B}$ whose irreducible components $D_{i}, i=1,2, \ldots, m^{\prime}$ are non-singular.

In this section we use the following notation freely.

$$
S_{j}=s_{j}(\mathcal{B}), \quad S=\sum_{j=1}^{N} S_{j} .
$$

Put

$$
\mathcal{F}_{N}(\mathcal{B})=\mathcal{F}_{N} \otimes_{\mathbf{C}} \mathcal{O}_{\mathcal{B}}, \quad \mathcal{F}_{N}^{\dagger}(\mathcal{B})=\mathcal{O}_{\mathcal{B}} \otimes_{\mathbf{C}} \mathcal{F}_{N}^{\dagger}
$$


Definition 8.1. We define the subsheaf $\mathcal{V}_{\mathrm{ab}}^{\dagger}(\mathfrak{F})$ of $\mathcal{F}_{N}^{\dagger}(\mathcal{B})$ by the gauge conditions:

$$
\begin{aligned}
& \sum_{j=1}^{N}\langle\Phi| \psi\left[\omega_{j}\right]=0, \quad \text { for all } \omega \in \pi_{*}\left(\omega_{\mathcal{C} / \mathcal{B}}(* S)\right), \\
& \sum_{j=1}^{N}\langle\Phi| \bar{\psi}\left[f_{j}\right]=0, \quad \text { for all } f \in \pi_{*} \mathcal{O}_{\mathcal{C}}(* S) .
\end{aligned}
$$

where $\omega_{j}$ and $f_{j}$ are the Laurent expansion of $\omega$ and $f$ along $S_{j}$ with respect to the coordinate $\xi_{j}$.

The sheaf $\mathcal{V}_{\mathrm{ab}}^{\dagger}(\mathfrak{F})$ is called the sheaf of $(j=0)$ abelian vacua or the sheaf of abelian vacua of the family $\mathfrak{F}$. Similarly the sheaf $\mathcal{V}_{\mathrm{ab}}(\mathfrak{F})$ of $(j=0)$ dual abelian vacua of the family is defined by

$$
\mathcal{V}_{\mathrm{ab}}(\mathfrak{F})=\mathcal{F}_{N}(\mathcal{B}) / \mathcal{F}_{\mathrm{ab}}(\mathfrak{F}) .
$$

where $\mathcal{F}_{\text {ab }}(\mathfrak{F})$ is the $\mathcal{O}_{\mathcal{B}}$-submodule of $\mathcal{F}_{N}(\mathcal{B})$ given by $\mathcal{F}_{\text {ab }}(\mathfrak{F})=\mathcal{F}_{\text {ab }}^{0}(\mathfrak{F})+\mathcal{F}_{\text {ab }}^{1}(\mathfrak{F})$, where $\mathcal{F}_{\text {ab }}^{0}(\mathfrak{F})$ is the span of $\bar{\psi}[f] \mathcal{F}_{N}(\mathcal{B})$ for all $f \in \pi_{*} \mathcal{O}_{\mathcal{C}}(* S)$ and $\mathcal{F}_{\text {ab }}^{1}(\mathfrak{F})$ is the span of $\psi[\omega] \mathcal{F}_{N}(\mathcal{B})$ for all $\omega \in \pi_{*} \omega_{\mathcal{C} / \mathcal{B}}(* S)$.

Note that we have

$$
\mathcal{V}_{\mathrm{ab}}^{\dagger}(\mathfrak{F})=\underline{\operatorname{Hom}}_{\mathcal{O}_{\mathcal{B}}}\left(\mathcal{V}_{\mathrm{ab}}(\mathfrak{F}), \mathcal{O}_{\mathcal{B}}\right)
$$

Moreover, by the right exactness of the tensor product we have that

$$
\mathcal{V}_{\mathrm{ab}}(\mathfrak{F}) \otimes_{\mathcal{O}_{\mathcal{B}}} \mathcal{O}_{\mathcal{B}, b} / \mathfrak{m}_{b} \cong \mathcal{V}_{\mathrm{ab}}(\mathfrak{F}(b))
$$

Theorem 8.1 ([2, Theorem 5.2]). The sheaves $\mathcal{V}_{\mathrm{ab}}(\mathfrak{F})$ and $\mathcal{V}_{\mathrm{ab}}^{\dagger}(\mathfrak{F})$ are invertible $\mathcal{O}_{\mathcal{B}}$-modules. They are dual to each other.

8.2. The line bundle of abelian vacua over Teichmüller space. Let $\mathfrak{F}_{i}, i=1,2$ be two families of stable and saturated pointed Riemann surfaces with formal neighbourhoods. Assume we have a morphism of families (not necessarily preserving sections nor formal coordinates)

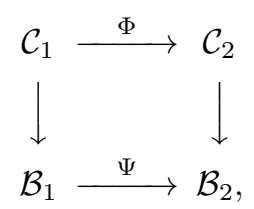

which is a fiberwise biholomorphism.

Let now $\mathfrak{F}_{0}=\left(\mathcal{C}_{1} \rightarrow \mathcal{B}_{1} ; \vec{s}_{0}, \vec{\eta}_{0}\right)$ be obtained from $\mathfrak{F}_{1}$, by replacing $\left(\vec{s}_{1}, \vec{\eta}_{1}\right)$ by $\left(\vec{s}_{0}, \vec{\eta}_{0}\right)$ such that $\vec{s}_{0}\left(\mathcal{B}_{1}\right)$ is disjoint from $\vec{s}_{1}\left(\mathcal{B}_{1}\right)$ and from $\overrightarrow{\tilde{s}}_{2}\left(\mathcal{B}_{1}\right)$, where $\Phi \overrightarrow{\tilde{s}}_{2}=\vec{s}_{2} \Psi$. The propagation of vacua isomorphism induces an isomorphism between $\mathcal{V}_{\mathrm{ab}}^{\dagger}\left(\mathfrak{F}_{0}\right)$ and $\mathcal{V}_{\mathrm{ab}}^{\dagger}\left(\mathfrak{F}_{1}\right)$. Furthermore the propagation of vacua induces an isomorphism between $\mathcal{V}_{\mathrm{ab}}^{\dagger}\left(\mathfrak{F}_{0}\right)$ and $\mathcal{V}_{\mathrm{ab}}^{\dagger}\left(\tilde{\mathfrak{F}}_{2}\right)$, where $\tilde{\mathfrak{F}}_{2}=\left(\mathcal{C}_{1} \rightarrow \mathcal{B}_{1} ; \overrightarrow{\tilde{s}}_{2}, \overrightarrow{\tilde{\eta}}_{2}\right)$ and $\Phi \overrightarrow{\tilde{\eta}}_{2}=\Psi^{*} \vec{\eta}_{2}$. The identity on $\mathcal{F}_{N}\left(\mathcal{B}_{1}\right)$ induces an isomorphism between $\mathcal{V}_{\text {ab }}^{\dagger}\left(\tilde{\mathcal{F}}_{2}\right)$ and $\mathcal{V}_{\text {ab }}^{\dagger}\left(\Psi^{*}\left(\mathcal{F}_{2}\right)\right)$. Composing these with the pull back isomorphism just as in the non-abelian case, we arrive at the following proposition. 
Proposition 8.1. We get an induced bundle morphism

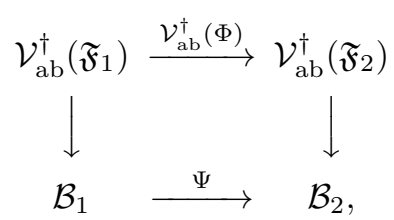

determined as above. Moreover, composition of such family morphisms goes to composition of the induced bundle morphisms.

Suppose now that we have two families $\mathfrak{F}_{i}, i=1,2$ over $\Sigma$ with the property that they have the same image $\tilde{\Psi}_{\mathfrak{F}_{1}}\left(\mathcal{B}_{1}\right)=\tilde{\Psi}_{\mathfrak{F}_{2}}\left(\mathcal{B}_{2}\right)$ in Teichmüller space $\mathcal{T}_{\Sigma}$ and that $\mathfrak{F}_{2}$ is a good family with respect to $\Sigma$. For such a pair of families there exists by Proposition 3.2 a unique fiber preserving biholomorphism $\Phi_{12}: \mathcal{C}_{1} \rightarrow \mathcal{C}_{2}$ covering $\Psi_{\mathfrak{F}_{2}}^{-1} \Psi_{\mathfrak{F}_{1}}$ such that $\Phi_{\mathfrak{F}_{2}}^{-1} \Phi_{12} \Phi_{\mathfrak{F}_{1}}: Y \rightarrow Y$ is isotopic to $\Psi_{\mathfrak{F}_{2}}^{-1} \Psi_{\mathfrak{F}_{1}} \times$ Id through such fiber preserving maps.

By Theorem 8.1 we have that $\mathcal{V}_{\mathrm{ab}}^{\dagger}\left(\mathfrak{F}_{i}\right)$ are holomorphic line bundles over $\mathcal{B}_{i}$. By Proposition 8.1 we get induced a glueing isomorphism

$$
\mathcal{V}_{\mathrm{ab}}^{\dagger}\left(\Phi_{12}\right): \mathcal{V}_{\mathrm{ab}}^{\dagger}\left(\mathfrak{F}_{1}\right) \rightarrow \mathcal{V}_{\mathrm{ab}}^{\dagger}\left(\mathfrak{F}_{2}\right)
$$

Definition 8.2. Let $\Sigma$ be a closed oriented smooth surface. We now define a line bundle $\mathcal{V}_{\mathrm{ab}}^{\dagger}=$ $\mathcal{V}_{\text {ab }}^{\dagger}(\Sigma, P)$ over Teichmüller space $\mathcal{T}_{\Sigma}$ using the cover $\left\{\tilde{\Psi}_{\mathfrak{F}}(\mathcal{B})\right\}$, where $\mathfrak{F}$ runs over the stable and saturated good families of pointed Riemann surfaces with formal neighbourhoods over $\Sigma$. Over $\tilde{\Psi}_{\mathfrak{F}}(\mathcal{B})$ we specify the line bundle as $\left(\tilde{\Psi}_{\mathfrak{F}}^{-1}\right)^{*} \mathcal{V}_{\text {ab }}^{\dagger}(\mathfrak{F})$. On overlaps of the image of two good families, we use the glueing isomorphism $\mathcal{V}_{\mathrm{ab}}^{\dagger}\left(\Phi_{12}\right)$ to glue the corresponding bundles together.

We obviously have the following

Proposition 8.2. For any stable and saturated family $\tilde{\mathfrak{F}}$ of pointed Riemann surfaces with formal neighbourhoods over $\Sigma$ we have a preferred isomorphism

$$
\tilde{\Upsilon}_{\tilde{\mathfrak{F}}}: \mathcal{V}_{\mathrm{ab}}^{\dagger}(\tilde{\mathfrak{F}}) \rightarrow \tilde{\Psi}_{\tilde{\mathfrak{F}}}^{*} \mathcal{V}_{\mathrm{ab}}^{\dagger}(\Sigma)
$$

induced by the transformation isomorphism between $\mathcal{V}_{\mathrm{ab}}^{\dagger}(\tilde{\mathfrak{F}})$ and $\mathcal{V}_{\mathrm{ab}}^{\dagger}(\mathfrak{F})$, for good families $\mathfrak{F}$ of pointed Riemann surfaces with formal neighbourhoods over $\Sigma$ such that $\tilde{\Psi}_{\mathfrak{F}}(\mathcal{B})$ intersect $\tilde{\Psi}_{\tilde{\mathfrak{F}}}\left(\mathcal{B}^{\prime}\right)$ nonempty.

Suppose now $f: \Sigma_{1} \rightarrow \Sigma_{2}$ is a morphism of surfaces. Then of course $f$ induces a biholomorphism $f^{*}$ from $\mathcal{T}_{\Sigma_{1}}$ to $\mathcal{T}_{\Sigma_{2}}$. Let now $\mathfrak{F}_{1}$ be a good family of stable pointed Riemann surfaces with formal neighbourhoods over $\Sigma_{1}$. Then by composing with $f^{-1} \times$ Id we get a good family $\mathfrak{F}_{2}$ of stable pointed Riemann surfaces with formal neighbourhoods over $\Sigma_{2}$ over the same base $\mathcal{B}_{1}$. The identity morphism on $\mathcal{F}_{N}\left(\mathcal{B}_{1}\right)$ then induces a morphism $\mathcal{V}_{\mathrm{ab}}^{\dagger}(f): \mathcal{V}_{\mathrm{ab}}^{\dagger}\left(\mathfrak{F}_{1}\right) \rightarrow \mathcal{V}_{\mathrm{ab}}^{\dagger}\left(\mathfrak{F}_{2}\right)$ which covers the identity on the base. This is precisely the morphism induced from the morphism of families $\Phi_{f}=f \times \operatorname{Id}: \mathfrak{F}_{1} \rightarrow \mathfrak{F}_{2}$ by Proposition 8.1. This in turn induces a morphism $\mathcal{V}_{\mathrm{ab}}^{\dagger}(f):\left(\tilde{\Psi}_{\mathfrak{F}_{1}}^{-1}\right)^{*}\left(\mathcal{V}_{\mathrm{ab}}^{\dagger}\left(\mathfrak{F}_{1}\right)\right) \rightarrow\left(\tilde{\Psi}_{\mathfrak{F}_{2}}^{-1}\right)^{*}\left(\mathcal{V}_{\mathrm{ab}}^{\dagger}\left(\mathfrak{F}_{2}\right)\right)$ which covers $f^{*}: \tilde{\Psi}_{\mathfrak{F}_{1}}\left(B_{1}\right) \rightarrow \tilde{\Psi}_{\mathfrak{F}_{2}}\left(B_{1}\right)$. 
Proposition 8.3. The above construction provides a well defined lift of $f^{*}: \mathcal{T}_{\Sigma_{2}} \rightarrow \mathcal{T}_{\Sigma_{1}}$ to a morphism $\mathcal{V}_{\mathrm{ab}}^{\dagger}(f): \mathcal{V}_{\mathrm{ab}}^{\dagger}\left(\Sigma_{1}\right) \rightarrow \mathcal{V}_{\mathrm{ab}}^{\dagger}\left(\Sigma_{2}\right)$ which behaves well under compositions.

The proof is exactly the same as the proof of Proposition 5.4 .

9. The CONnection in the line Bundle of Abelian VACUA OVER Teichmüller SPACE.

Let us use the same notation as in $\S 6.1$. Let $\mathfrak{F}$ be a family of stable and saturated pointed Riemann surfaces with formal neighbourhoods on $(\Sigma, P)$. For an element $\vec{\ell}=\left(\ell_{1}, \ldots, \ell_{N}\right)$ in

$$
\mathcal{L}(\mathfrak{F}):=\bigoplus_{j=1}^{N} \mathcal{O}_{\mathcal{B}}\left[\xi_{j}^{-1}\right] \frac{d}{d \xi_{j}}
$$

the action $D(\vec{\ell})$ on $\mathfrak{F}$ is defined by

$$
D(\vec{\ell})(F \otimes|u\rangle)=\theta(\vec{\ell})(F) \otimes|u\rangle-F \cdot\left(\sum_{j=1}^{N} \rho_{j}\left(T\left[\ell_{j}\right]\right)\right)|u\rangle
$$

where

$$
F \in \mathcal{O}_{\mathcal{B}}, \quad|u\rangle \in \mathcal{F}_{N}
$$

and

$$
T[\ell]=\operatorname{Res}_{z=0}(T(z) \ell(z) d z), .
$$

Here $T(z)$ is the energy-momentum tensor $\mathrm{T}$ of spin $j=0$ bc ghost system. Then the action has the similar properties as those of Proposition 6.1. We define the dual action of $\mathcal{L}(\mathfrak{F})$ on $\mathcal{F}_{N}^{\dagger}(\mathcal{B})$ by

$$
D(\vec{\ell})(F \otimes\langle\Phi|)=\theta(\vec{\ell})(F) \otimes\langle\Phi|+\sum_{j=1}^{N} F \cdot\langle\Phi| \rho_{j}\left(T\left[\ell_{j}\right]\right) .
$$

where

$$
F \in \mathcal{O}_{\mathcal{B}}, \quad\langle\Phi| \in \mathcal{F}_{N}^{\dagger}(\mathcal{B}) .
$$

Then, for any $|u\rangle \in \mathcal{F}_{N}(\mathcal{B})$ and $\langle\Phi| \in \mathcal{F}_{N}^{\dagger}(\mathcal{B})$, we have

$$
\{D(\vec{\ell})\langle\Phi|\}|\widetilde{\Phi}\rangle+\langle\Phi|\{D(\vec{\ell})|\widetilde{\Phi}\rangle\}=\theta(\vec{\ell})\langle\Phi \mid \widetilde{\Phi}\rangle .
$$

Now the operator $D(\vec{\ell})$ acts on $\mathcal{V}_{\mathrm{ab}}(\mathfrak{F})$.

Proposition 9.1 ([2, Proposition 4.2]). For any $\vec{\ell} \in \mathcal{L}(\mathfrak{F})$ we have

$$
D(\vec{\ell})\left(\mathcal{F}_{\mathrm{ab}}(\mathfrak{F})\right) \subset \mathcal{F}_{\mathrm{ab}}(\mathfrak{F}) .
$$

Hence, $D(\vec{\ell})$ operates on $\mathcal{V}_{\mathrm{ab}}(\mathfrak{F})$. Moreover, it is a first order differential operator, if $\theta(\vec{\ell}) \neq 0$. 
Now choose a meromorphic bidifferential

$$
\omega \in H^{0}\left(\mathcal{C} \times{ }_{\mathcal{B}} \mathcal{C}, \omega_{\mathcal{C} \times{ }_{\mathcal{B}} \mathcal{C}}(2 \Delta)\right)
$$

defined by (42). Put

$$
b_{\omega}(\vec{\ell})=\sum_{j=1}^{N} \operatorname{Res}_{\xi_{j}=0}\left(\ell_{j}\left(\xi_{j}\right) S_{\omega}\left(\xi_{j}\right) d \xi_{j}\right)
$$

where $S_{\omega}$ is the projective connection defined by (45). Then this defines an $\mathcal{O}_{\mathcal{B}}$-module homomorphism

$$
b_{\omega}: \mathcal{L}(\mathfrak{F}) \rightarrow \mathcal{O}_{\mathcal{B}}
$$

and if $\theta(\vec{\ell})=0$ then we have that

$$
\langle\Phi|\{D(\vec{\ell})|u\rangle\}=\frac{1}{6} b_{\omega}(\vec{\ell})\langle\Phi \mid u\rangle .
$$

For a vector field $X$ on $\mathcal{B}$ choose $\vec{\ell} \in \mathcal{L}(\mathfrak{F})$ such that $\theta(\vec{\ell})=X$. Then the connection on $\mathcal{V}_{\text {ab }}^{\dagger}(\mathfrak{F})$ is defined by

$$
\nabla_{X}^{(\omega)}(\langle\Phi|)=D(\vec{\ell})(\langle\Phi|)+\frac{1}{6} b_{\omega}(\vec{\ell})(\langle\Phi|),
$$

for $\langle\Phi| \in \mathcal{V}_{\mathrm{ab}}^{\dagger}(\mathfrak{F})$. This is well-defined and (64) is independent of the choice of $\vec{\ell} \in \mathcal{L}(\mathfrak{F})$ with $\theta(\vec{\ell})=X$. Just like for the non-abelian conformal field theory (see for example [19], section 5) we can prove the following theorem.

Theorem 9.1 ([2, Theorem 4.2]). The operator $\nabla^{(\omega)}$ defines a projectively flat holomorphic connection of the sheaves $\mathcal{V}_{\mathrm{ab}}(\mathfrak{F})$ and $\mathcal{V}_{\mathrm{ab}}^{\dagger}(\mathfrak{F})$. Moreover, the connection has a regular singularity along the locus $D \subset \mathcal{B}$ which is the locus of the singular curves. The connection $\nabla^{(\omega)}$ depends on the choice of bidifferential $\omega$ and if we choose another bidifferential $\omega^{\prime}$ then there exists a holomorphic one-form $\phi_{\omega, \omega}$ on $\mathcal{B}$ such that

$$
\nabla_{X}^{(\omega)}-\nabla_{X}^{\left(\omega^{\prime}\right)}=\frac{1}{6}\left\langle\phi_{\omega, \omega^{\prime}}, X\right\rangle
$$

Moreover, the curvature form $R$ of $\nabla_{X}^{(\omega)}$ is given by

$$
R(X, Y)=\frac{1}{6}\left\{b_{\omega}(\vec{n})-X\left(b_{\omega}(\vec{m})\right)+Y\left(b_{\omega}(\vec{\ell})\right)-\sum_{j=1}^{N} \operatorname{Res}_{\xi_{j}=0}\left(\frac{d^{3} \ell_{j}}{d \xi_{j}} m_{j} d \xi_{j}\right)\right\}
$$

where $\left.X, Y \in \Theta_{\mathcal{C} / \mathcal{B}}(* S)\right), \vec{\ell}, \vec{m} \in \mathcal{L}(\mathfrak{F})$ with $X=\theta(\vec{\ell}), Y=\theta(\vec{m})$, and $\vec{n} \in \mathcal{L}(\mathfrak{F})$ is defined by $\vec{n}=[\vec{\ell}, \vec{m}]_{d}($ see $(39))$. 
Corollary 9.1. Let $\mathfrak{F}$ be a family of stable and saturated pointed Riemann surfaces with formal neighbourhoods on $(\Sigma, P)$. If we use the same bidifferential $\omega$ to define the connections on the bundle of vacua and the line bundle of abelian vacua on $\mathfrak{F}$, then we have

$$
R^{\omega}(X, Y)=\frac{c_{v}}{2} R(X, Y) \otimes \operatorname{Id}
$$

Proposition 9.2. Let $\mathfrak{F}$ be a family of stable and saturated pointed Riemann surfaces with formal neighbourhoods on $(\Sigma, P)$ and choose a symplectic basis $\left(\alpha_{1}, \ldots, \alpha_{g}, \beta_{1}, \ldots, \beta_{g}\right)$ of $H_{1}(\Sigma, \mathbb{Z})$. Let $\omega \in H^{0}\left(\mathcal{C} \times{ }_{\mathcal{B}} \mathcal{C}, \omega_{\mathcal{C} \times{ }_{\mathcal{B}} \mathcal{B} B}(2 \Delta)\right)$ be the normalized symmetric bidifferential determined by this data. Then there is the connection $\tilde{\nabla}^{(\omega)}$ in the bundle $\mathcal{V}_{\mathrm{ab}}^{\dagger}(\mathfrak{F})$ whose $(1,0)$-part is given by formula (4.23) in [2] and whose $(0,1)$-part is just the $\bar{\partial}$-operator in this holomorphic line-bundle. The curvature of this connection is given by the formula (66).

Proof. By the definition of $\tilde{\nabla}^{(\omega)}$ and the definition (63) of $b_{\omega}$, we see that the $(1,1)$ and $(0,2)$-part of the curvature is zero.

Suppose now that we have two good families $\mathfrak{F}_{i}, i=1,2$ with the property that they have the same image $\tilde{\Psi}_{\mathfrak{F}_{1}}\left(\mathcal{B}_{1}\right)=\tilde{\Psi}_{\mathfrak{F}_{2}}\left(\mathcal{B}_{2}\right)$ in Teichmüller space $\mathcal{T}_{\Sigma}$. For such a pair of families there exists by Proposition 3.2 a unique fiber preserving biholomorphism $\Phi_{12}: \mathcal{C}_{1} \rightarrow \mathcal{C}_{2}$ covering $\Psi_{\mathfrak{F}_{2}}^{-1} \Psi_{\mathfrak{F}_{1}}$ such that $\Phi_{\mathfrak{F}_{2}}^{-1} \Phi_{12} \Phi_{\mathfrak{F}_{1}}:(Y, P) \rightarrow(Y, P)$ is isotopic to $\Psi_{\mathfrak{F}_{2}}^{-1} \Psi_{\mathfrak{F}_{1}} \times$ Id.

Lemma 9.1. Let $\tilde{\nabla}_{i}^{(\omega)}$ be the connection in $\mathcal{V}_{\mathrm{ab}}^{\dagger}\left(\mathfrak{F}_{i}\right)$ described in Proposition 9.2. Then we have that

$$
\mathcal{V}_{\mathrm{ab}}^{\dagger}\left(\Phi_{12}\right)^{*}\left(\tilde{\nabla}_{2}^{(\omega)}\right)=\tilde{\nabla}_{1}^{(\omega)}
$$

This follows from Theorem 4.4 above, by the same argument as in the non-abelian case.

Theorem 9.2. Let $\Sigma$ be a closed oriented surface and let $(\vec{\alpha}, \vec{\beta})=\left(\alpha_{1}, \ldots, \alpha_{g}, \beta_{1}, \ldots, \beta_{g}\right)$ be a symplectic basis of $H_{1}(\Sigma, \mathbb{Z})$. There is a unique connection $\tilde{\nabla}^{(\vec{\alpha}, \vec{\beta})}=\tilde{\nabla}^{(\vec{\alpha}, \vec{\beta})}(\Sigma, P)$ in the bundle $\mathcal{V}_{\mathrm{ab}}^{\dagger}(\Sigma, P)$ over $\mathcal{T}_{\Sigma}$ with the property that for any good family $\mathfrak{F}$ of stable pointed Riemann surfaces with formal neighbourhoods over $\Sigma$ we have that

$$
\tilde{\Psi}_{\mathfrak{F}}^{*}\left(\tilde{\nabla}^{(\vec{\alpha}, \vec{\beta})}\right)=\tilde{\nabla}^{(\omega)} .
$$

In particular the connection is compatible with the holomorphic line-bundle structure on $\mathcal{V}_{\mathrm{ab}}^{\dagger}(\Sigma, P)$. The curvature is of type $(2,0)$ as stated in Proposition 9.2.

If we act on the symplectic basis $(\vec{\alpha}, \vec{\beta})$ by an element $\Lambda=\left(\begin{array}{cc}A & B \\ C & D\end{array}\right) \in S p(g, \mathbb{Z})$ so as to obtain $\Lambda(\vec{\alpha}, \vec{\beta})$, as defined by (43), then

$$
\tilde{\nabla}^{\Lambda(\vec{\alpha}, \vec{\beta})}-\tilde{\nabla}^{(\vec{\alpha}, \vec{\beta})}=\frac{1}{2} \Pi^{*}(d \log \operatorname{det}(C \tau+D)),
$$


where $\Pi$ is the period mapping of holomorphic one-forms form the base space of $\mathfrak{F}$ to the Siegel upperhalf plane of degree $g$. If $f: \Sigma_{1} \rightarrow \Sigma_{2}$ is an orientation preserving diffeomorphism of surfaces which maps the symplectic basis $\left(\vec{\alpha}^{(1)}, \vec{\beta}^{(1)}\right)$ of $H_{1}\left(\Sigma_{1}, \mathbb{Z}\right)$ to the symplectic basis $\left(\vec{\alpha}^{(2)}, \vec{\beta}^{(2)}\right)$ of $H_{1}\left(\Sigma_{2}, \mathbb{Z}\right)$ then we have that

$$
\mathcal{V}_{\mathrm{ab}}^{\dagger}(f)^{*}\left(\tilde{\nabla}^{\left(\vec{\alpha}^{(2)}, \vec{\beta}^{(2)}\right)}\right)=\tilde{\nabla}^{\left(\vec{\alpha}^{(1)}, \vec{\beta}^{(1)}\right)} .
$$

Proof. The existence is a consequence of Lemma 9.1. The transformation law (68) follows from the above Theorem 9.1 .

Proposition 9.3. For any stable and saturated family $\tilde{\mathfrak{F}}$ of pointed Riemann surfaces with formal neighbourhoods over $\Sigma$ the preferred isomorphism

$$
\tilde{\Upsilon}_{\tilde{\mathfrak{F}}}: \mathcal{V}_{\mathrm{ab}}^{\dagger}(\tilde{\mathfrak{F}}) \rightarrow \tilde{\Psi}_{\tilde{\mathfrak{F}}}^{*} \mathcal{V}_{\mathrm{ab}}^{\dagger}(\Sigma)
$$

given by Proposition 8 .2 preserves connections and is compatible with the lift $\mathcal{V}_{\mathrm{ab}}^{\dagger}(f)$.

This follows directly from Lemma 9.1 and Theorem 9.2 .

10. The PREFERRED NON-VANishing SECTION OF THE BUNDLE OF ABELIAN VACUA.

Let $\mathfrak{X}=(C ; Q ; \xi)$ be a one-pointed smooth curve of genus $g$ with a formal neighbourhood. We shall show that if we fix a symplectic basis $(\vec{\alpha}, \vec{\beta})=\left(\alpha_{1}, \ldots, \alpha_{g}, \beta_{1}, \ldots, \beta_{g}\right)$ of $H_{1}(C, \mathbf{Z})$, then there is a canonical preferred non-zero vector $\left\langle\omega\left(\mathfrak{X},(\{\alpha, \beta\}) \mid \in \mathcal{V}_{\mathrm{ab}}^{\dagger}(\mathfrak{X})\right.\right.$. Let us choose a normalized basis $\left\{\omega_{1}, \ldots, \omega_{g}\right\}$ of holomorphic one-forms on $C$ which is characterized by

$$
\int_{\beta_{i}} \omega_{j}=\delta_{i j}, \quad 1 \leq i, j \leq g
$$

The period matrix is given by

$$
\tau=\left(\tau_{i j}\right), \quad \tau_{i j}=\int_{\alpha_{i}} \omega_{j} .
$$

Now the numbers $I_{n}^{i}, n=1,2, \ldots, i=1, \ldots g$ are defined by

$$
\omega_{i}=\left(\sum_{n=1}^{\infty} I_{n}^{i} \xi^{n-1}\right) d \xi
$$

Note that the numbers $I_{n}^{i}$ depend on the symplectic basis $(\vec{\alpha}, \vec{\beta})$ and the formal neighbourhood $\xi$.

For a positive integer $n \geq 1$ let $\omega_{Q}^{(n)}$ be a meromorphic one-form on $C$ which has a pole of order $n+1$ at $Q$ and holomorphic elsewhere such that

$$
\begin{aligned}
\int_{\alpha_{i}} \omega_{Q}^{(n)} & =-\frac{2 \pi \sqrt{-1} I_{n}^{i}}{n}, \quad \int_{\beta_{i}} \omega_{Q}^{(n)}=0, \quad 1 \leq i \leq g \\
\omega_{Q}^{(n)} & =\left(\frac{1}{\xi^{n+1}}+\sum_{m=1}^{\infty} q_{n, m} \xi^{m-1}\right) d \xi .
\end{aligned}
$$


These conditions uniquely determine $\omega_{Q}^{(n)}$. Note that the second equality of (170) and (71) imply the first equality of (70). The preferred element $\langle\omega(\mathfrak{X},\{\alpha, \beta\})| \in \mathcal{V}_{\mathrm{ab}}^{\dagger}(\mathfrak{X})$ is defined by

$$
\langle\omega(\mathfrak{X},\{\alpha, \beta\})|=\cdots e\left(\omega_{g+2}\right) \wedge e\left(\omega_{g+1}\right) \wedge e\left(\omega_{g}\right) \wedge \cdots \wedge e\left(\omega_{1}\right),
$$

where

$$
\omega_{g+n}=\omega_{Q}^{(n)}
$$

For details see Lemma 3.1 and its proof of [2]. We call $\left\{\omega_{n}\right\}, n=1,2, \ldots$ a normalized basis for $\mathfrak{X}$. Note that the normalized basis depends on the choice of a symplectic basis of $H_{1}(C, \mathbf{Z})$ and the coordinate $\xi$.

Theorem 10.1 ([2, Theorem 6.2]). For $h(\xi) \in \mathcal{D}_{+}^{0}$ put $\mathfrak{X}_{h}=\{C ; Q ; \eta=h(\xi)\}$. Then

$$
\langle\omega(\mathfrak{X},\{\alpha, \beta\})| G[h]=\left\langle\omega\left(\mathfrak{X}_{h},\{\alpha, \beta\}\right)\right|,
$$

where $G[h]: \mathcal{V}_{\mathrm{ab}}^{\dagger}(\mathfrak{X}) \rightarrow \mathcal{V}_{\mathrm{ab}}^{\dagger}\left(\mathfrak{X}_{h}\right)$ is the canonical isomorphism given in Proposition 7.2

Theorem $10.2\left(\left[2\right.\right.$, Theorem 6.3]). Let $\left(\alpha_{1}, \ldots \alpha_{g}, \beta_{1}, \ldots, \beta_{g}\right)$ and $\left(\widetilde{\alpha}_{1}, \ldots \widetilde{\alpha}_{g}, \widetilde{\beta}_{1}, \ldots, \widetilde{\beta}_{g}\right)$ be symplectic bases of $H^{1}(C, \mathbf{Z})$ of the non-singular curve $C$. Assume that $\left\{\beta_{1}, \ldots, \beta_{g}\right\}$ and $\left\{\widetilde{\beta}_{1}, \ldots, \widetilde{\beta}_{g}\right\}$ span the same Lagrangian sublattice in $H^{1}(C, \mathbf{Z})$. Then

$$
\langle\omega(\mathfrak{X},\{\alpha, \beta\})|=\operatorname{det} U\langle\omega(\mathfrak{X},\{\widetilde{\alpha}, \widetilde{\beta}\})|,
$$

where $U \in G L(g, \mathbf{Z})$ is defined by

$$
\left(\begin{array}{c}
\widetilde{\beta}_{1} \\
\vdots \\
\widetilde{\beta}_{g}
\end{array}\right)=U\left(\begin{array}{c}
\beta_{1} \\
\vdots \\
\beta_{g}
\end{array}\right)
$$

Let $\{p, q\}$ be two smooth points on the curve $C$ with formal neighbourhoods $\xi$, $\eta$, respectively. Put $\mathfrak{X}_{0}=(C ; p, q ; \xi, \eta), \mathfrak{X}_{1}=(C ; p ; \xi), \mathfrak{X}_{2}=(C ; q ; \eta)$. Then the natural imbeddings

$$
\begin{aligned}
& \iota_{1}: \mathcal{F} \hookrightarrow \mathcal{F}_{2} \\
& |u\rangle \mapsto|u\rangle \otimes|0\rangle \\
& \iota_{2}: \mathcal{F} \hookrightarrow \mathcal{F}_{2} \\
& |u\rangle \mapsto|0\rangle \otimes|u\rangle
\end{aligned}
$$


induce canonical isomorphisms

$$
\begin{array}{cc} 
& \mathcal{V}_{\mathrm{ab}}^{\dagger}\left(\mathfrak{X}_{0}\right) \\
\iota_{1}^{*} \swarrow & \searrow^{\iota_{2}^{*}} \\
\mathcal{V}_{\mathrm{ab}}^{\dagger}\left(\mathfrak{X}_{1}\right) & \mathcal{V}_{\mathrm{ab}}^{\dagger}\left(\mathfrak{X}_{2}\right)
\end{array}
$$

by Theorem 7.4

Theorem 10.3 ([2, Theorem 6.4]). Under the above notation we have

$$
\iota_{2}^{*} \circ\left(\iota_{1}^{*}\right)^{-1}\left(\left\langle\omega\left(\mathfrak{X}_{1},\{\alpha, \beta\}\right)\right|\right)=\left\langle\omega\left(\mathfrak{X}_{2},\{\alpha, \beta\}\right)\right| .
$$

Let $\Sigma$ be a closed oriented surface. Assume first that $\Sigma$ is connected. As described above, the choice of a symplectic basis gives a preferred section in the line bundle of abelian vacua associated to any family of stable and saturated pointed Riemann surfaces with formal neighbourhoods over $\Sigma$. We have that

Theorem 10.4. Let $\mathfrak{F}$ be a family of stable and saturated pointed Riemann surfaces with formal neighbourhoods over $\Sigma$ and choose a symplectic basis $(\vec{\alpha}, \vec{\beta})$ of $H_{1}(\Sigma, \mathbb{Z})$. Then there is a preferred non-vanishing holomorphic section $s_{\mathfrak{F}}^{(\vec{\alpha}, \vec{\beta})}$ in the bundle $\mathcal{V}_{\mathrm{ab}}^{\dagger}(\mathfrak{F})^{\otimes 2}$ given by

$$
s_{\mathfrak{F}}^{(\vec{\alpha}, \vec{\beta})}(t)=\left(\left\langle\omega\left(\mathfrak{X}_{t},\{\alpha(t), \beta(t)\} \mid\right)^{\otimes 2} .\right.\right.
$$

If we act on the symplectic basis $(\vec{\alpha}, \vec{\beta})$ by an element $\Lambda=\left(\begin{array}{cc}\left(U^{t}\right)^{-1} & B \\ 0 & U\end{array}\right) \in S p(g, \mathbb{Z})$ in order to obtain $\Lambda(\vec{\alpha}, \vec{\beta})$ as described in (43), then

$$
s^{\Lambda(\vec{\alpha}, \vec{\beta})}=s^{(\vec{\alpha}, \vec{\beta})} .
$$

Proof. This is clear from Theorem 10.2, since we have $\operatorname{det} U= \pm 1$.

Thus the section $s_{\widetilde{F}}^{(\vec{\alpha}, \vec{\beta})}$ only really depends on the Lagrangian subspace $L=\operatorname{Span}\left\{\beta_{i}\right\}$ and we therefore denote it $s_{\mathfrak{F}}(L)$.

Suppose now that $\Sigma$ is not connected and that $\Sigma=\coprod_{i} \Sigma_{i}$ is the decomposition of $\Sigma$ into its connected components $\Sigma_{i}$. Let $\mathfrak{F}$ be a family of stable pointed Riemann surfaces with formal neighbourhoods over $\Sigma$. Let $\mathfrak{F}_{i}$ be the restriction of $\mathfrak{F}$ to $\Sigma_{i}$. Let $N_{i}$ be the number of sections of $\mathfrak{F}_{i}$ and $N=\sum_{i} N_{i}$ the number of sections of $\mathfrak{F}$. We obviously have the following lemma.

Lemma 10.1. The isomorphism $\mathcal{F}_{N} \cong \otimes_{i} \mathcal{F}_{N_{i}}$ induces an isomorphism of holomorphic line bundles

$$
\mathcal{V}_{\mathrm{ab}}^{\dagger}(\mathfrak{F}) \cong \otimes_{i} \mathcal{V}_{\mathrm{ab}}^{\dagger}\left(\mathfrak{F}_{i}\right)
$$

which is compatible with the connections. 
Suppose now that $(\vec{\alpha}, \vec{\beta})=\left(\left(\vec{\alpha}_{i}, \vec{\beta}_{i}\right)\right)$ is a symplectic basis of $H_{1}(\Sigma, \mathbb{Z})$. We then define the preferred section to be

$$
s_{\mathfrak{F}}^{(\vec{\alpha}, \vec{\beta})}=\otimes_{i} s_{\widetilde{F}_{i}}^{\left(\vec{\alpha}_{i}, \vec{\beta}_{i}\right)} .
$$

For the rest of this section $\Sigma$ is just any closed oriented surface.

Suppose now that we have two good families $\mathfrak{F}_{i}, i=1,2$ with the property that they have the same image $\tilde{\Psi}_{\widetilde{F}_{1}}\left(\mathcal{B}_{1}\right)=\tilde{\Psi}_{\widetilde{F}_{2}}\left(\mathcal{B}_{2}\right)$ in Teichmüller space $\mathcal{T}_{\Sigma}$. For such a pair of families there exists by Theorem 3.2 a unique fiber preserving biholomorphism $\Phi_{12}: \mathcal{C}_{1} \rightarrow \mathcal{C}_{2}$ covering $\Psi_{\mathfrak{F}_{2}}^{-1} \Psi_{\mathfrak{F}_{1}}$ such that $\Phi_{\mathfrak{F}_{2}}^{-1} \Phi_{12} \Phi_{\mathfrak{F}_{1}}: Y \rightarrow Y$ is isotopic to $\Psi_{\mathfrak{F}_{2}}^{-1} \Psi_{\mathfrak{F}_{1}} \times$ Id through fiber preserving diffeomorphisms.

Lemma 10.2. Let $s_{\mathfrak{F}_{i}}^{(\vec{\alpha}, \vec{\beta})}$ be the preferred sections of $\mathcal{V}_{\mathrm{ab}}^{\dagger}\left(\mathfrak{F}_{i}\right)^{\otimes 2}$ described in Theorem 10.4. Then we have that

$$
\mathcal{V}_{\mathrm{ab}}^{\dagger}\left(\Phi_{12}\right)^{\otimes 2}\left(s_{\mathfrak{F}_{1}}^{(\vec{\alpha}, \vec{\beta})}\right)=s_{\mathfrak{F}_{2}}^{(\vec{\alpha}, \vec{\beta})} .
$$

Proof. Since $\mathcal{V}_{\mathrm{ab}}^{\dagger}\left(\Phi_{12}\right)$ is induced by the propagation of vacua isomorphism and the coordinate change isomorphism, this lemma follows from Theorem 10.1 and 10.3

Theorem 10.5. Let $\Sigma$ be a closed oriented surface and let $(\vec{\alpha}, \vec{\beta})=\left(\alpha_{1}, \ldots, \alpha_{g}, \beta_{1}, \ldots, \beta_{g}\right)$ be a symplectic basis of $H_{1}(\Sigma, \mathbb{Z})$. Then there is a unique non-vanishing holomorphic section $s^{(\vec{\alpha}, \vec{\beta})}=$ $s_{\Sigma}^{(\vec{\alpha}, \vec{\beta})}$ in the bundle $\mathcal{V}_{\mathrm{ab}}^{\dagger}(\Sigma)^{\otimes 2}$ over $\mathcal{T}_{\Sigma}$ with the property that for any good family $\mathfrak{F}$ of stable pointed Riemann surfaces with formal neighbourhoods over $\Sigma$ we have that

$$
\left(\tilde{\Psi}_{\mathfrak{F}}^{*}\right)^{\otimes 2}\left(s^{(\vec{\alpha}, \vec{\beta})}\right)=s_{\mathfrak{F}}^{(\vec{\alpha}, \vec{\beta})} .
$$

The sections transforms according to the transformation rule 772. If $f: \Sigma_{1} \rightarrow \Sigma_{2}$ is an orientation preserving diffeomorphism of surfaces which maps the symplectic basis $\left(\vec{\alpha}^{(1)}, \vec{\beta}^{(1)}\right)$ of $H_{1}\left(\Sigma_{1}, \mathbb{Z}\right)$ to the symplectic basis $\left(\vec{\alpha}^{(2)}, \vec{\beta}^{(2)}\right)$ of $H_{1}\left(\Sigma_{2}, \mathbb{Z}\right)$ then we have that

$$
\left(\mathcal{V}_{\mathrm{ab}}^{\dagger}(f)^{*}\right)^{\otimes 2}\left(s_{\Sigma_{2}}^{\left(\vec{\alpha}^{(2)}, \vec{\beta}^{(2)}\right)}\right)=s_{\Sigma_{1}}^{\left(\vec{\alpha}^{(1)}, \vec{\beta}^{(1)}\right)} .
$$

Proof. The first part of this theorem follows from the above. Since the choice of basis of meromorphic 1 -forms with the relevant properties is natural w.r.t. morphisms of Riemann surfaces, we get that the preferred section transforms just like the bases does.

Likewise, we see that the section only depends on the Lagrangian subspace and we denote it therefore $s(L)=s_{\Sigma}(L)$.

Proposition 10.1. For any stable and saturated family $\tilde{\mathfrak{F}}$ of pointed Riemann surfaces with formal neighbourhoods over $\Sigma$ the preferred isomorphism

$$
\tilde{\Upsilon}_{\tilde{\mathfrak{F}}}^{\otimes 2}: \mathcal{V}_{\mathrm{ab}}^{\dagger}(\tilde{\widetilde{F}})^{\otimes 2} \rightarrow \tilde{\Psi}_{\tilde{\mathfrak{F}}}^{*} \mathcal{V}_{\mathrm{ab}}^{\dagger}(\Sigma)^{\otimes 2}
$$


given by Proposition 8.2 preserves the preferred sections.

This Follows from Lemma 10.2

\section{The GeOMEtric CONSTRUCTION OF the MOdUlar FUnCTOR.}

For the convenience of the reader, let us summarize the results of the sheaf of vacua constructions over Teichmüller spaces of pointed surfaces obtained in non-abelian case in sections 4.6 to 6 and in the abelian case in sections 7 to 10

Theorem 11.1. Let $(\Sigma, P, \lambda)$ be a stable and saturated labeled pointed surface.

- The sheaf of vacua construction (see Definition [5.2) yields a vector bundle $\mathcal{V}_{\lambda}^{\dagger}=\mathcal{V}_{\lambda}^{\dagger}(\Sigma, P)$ over the Teichmüller space $\mathcal{T}_{(\Sigma, P)}$ of $(\Sigma, P)$ whose fiber at a complex structure $\mathbf{C}$ on $(\Sigma, P)$ is identified (via the isomorphism given in Proposition [5.2) with the space of vacua $\mathcal{V}_{\lambda}^{\dagger}(\mathbf{C})$ as defined in Definition 4.5 .

- For each symplectic basis of $H_{1}(\Sigma, \mathbb{Z})$, we get induced a connection in $\mathcal{V}_{\lambda}^{\dagger}$ over $\mathcal{T}_{(\Sigma, P)}$. Any two of these connections differ by a global scalar-value 1-form on $\mathcal{T}_{(\Sigma, P)}$. See Theorem 6.3 .

- Each of these connections is projectively flat and their curvature are described in details in Theorem 6.2 and 9.1 .

- There is a natural lift of morphisms of pointed surfaces to these bundles covering induced biholomorphisms between Teichmüller spaces, which preserves compositions. See Proposition 5.4 .

- A morphism of pointed surfaces transforms these connections according to the way it transforms symplectic bases of the first homology. See Theorem 6.2.

Remark 11.1. If we choose a Lagrangian subspace $L$ of $H_{1}(\Sigma, \mathbb{Z})$ and constrain the symplectic basis $\left(\alpha_{i}, \beta_{i}\right)$ of $H_{1}(\Sigma, \mathbb{Z})$ such that $L=\operatorname{Span}\left\{\beta_{i}\right\}$ then we see from the transformation laws in Theorem 6.2. that we get a connection in $\mathcal{V}_{\lambda}^{\dagger}$ which depends only on $L$.

Since the connections in the vector bundle $\mathcal{V}_{\lambda}^{\dagger}$ are only projectively flat, we need a 1-dimensional theory with connections, whose curvature after taking tensor products, can cancel this curvature and result in a bundle with a flat connection. There are obstructions to doing this mapping class group equivariantly, so we expect to see central extension of the mapping class groups occurring. As we shall see below, this is exactly what happens, when one extracts the necessary root of the abelian theory treated in 2, so as to get the right scaling of the curvature. Again, the following theorem summarizes the results and constructions, now in the abelian case treated in section 7 to 10 .

Theorem 11.2. Let $\Sigma$ be a closed oriented surface.

- The sheaf of abelian vacua construction (see Definition 8.2) yields a line bundle $\mathcal{V}_{\mathrm{ab}}^{\dagger}=\mathcal{V}_{\mathrm{ab}}^{\dagger}(\Sigma$ ) over the Teichmüller space $\mathcal{T}_{\Sigma}$ of $\Sigma$, whose fiber at a complex structure $\mathbf{C}$ on $\Sigma$ is identified 
(via the isomorphism given in Proposition 8.2) with the space of abelian vacua $\mathcal{V}_{\mathrm{ab}}^{\dagger}(\mathbf{C})$ as defined in Definition 7.4.

- For each symplectic basis of $H_{1}(\Sigma, \mathbb{Z})$, we get induced a holomorphic connection in $\mathcal{V}_{\mathrm{ab}}^{\dagger}$ over $\mathcal{T}_{\Sigma}$ (see Theorem 9.2). The difference between the connections associated to two different basis's is the global scalar-value 1-form on $\mathcal{T}_{\Sigma}$ given in (68).

- The curvature of each of these connections are described in Proposition 9.2.

- For each symplectic basis of $H_{1}(\Sigma, \mathbb{Z})$, we also get a preferred non-vanishing section of $\left(\mathcal{V}_{\mathrm{ab}}^{\dagger}\right)^{\otimes 2}$ as specified in Theorem 10.5. The transformation formula (72) states how the preferred sections transforms under change of the symplectic basis of $H_{1}(\Sigma, \mathbb{Z})$.

- There is a natural lift of morphisms of surfaces to these bundles covering induced biholomorphisms between Teichmüller space, which preserves compositions. See Proposition 8.3.

- A morphism of surfaces transforms these connections and the preferred sections according to the way it transforms symplectic bases of the first homology. See Theorem 9.1 and 10.5 .

Remark 11.2. If we choose a Lagrangian subspace $L$ of $H_{1}(\Sigma, \mathbb{Z})$ and constrain the symplectic basis $\left(\alpha_{i}, \beta_{i}\right)$ of $H_{1}(\Sigma, \mathbb{Z})$ such that $L=\operatorname{Span}\left\{\beta_{i}\right\}$ then we see from the transformation laws in Theorem 10.5, that we get a preferred non-vanishing section $s=s(L)$ and a connection in $\left(\mathcal{V}_{\mathrm{ab}}^{\dagger}\right)^{\otimes 2}$ which only depends on $L$.

From the discussion of the curvatures of the connections in $\mathcal{V}_{\lambda}^{\dagger}$ and $\mathcal{V}_{\mathrm{ab}}^{\dagger}$, i.e. by comparing the curvature formula (67), it is clear that the root of $\left(\mathcal{V}_{\mathrm{ab}}^{\dagger}\right)^{\otimes 2}$ we are seeking is $c_{v}$. The following theorem provided us with such a root.

Theorem 11.3. For any marked surface $\boldsymbol{\Sigma}=(\Sigma, L)$ there exists a line bundle, which we denoted $\left(\mathcal{V}_{\mathrm{ab}}^{\dagger}\right)^{-\frac{1}{2} c_{v}}(L)=\left(\mathcal{V}_{\mathrm{ab}}^{\dagger}\right)^{-\frac{1}{2} c_{v}}(\boldsymbol{\Sigma})$, over $\mathcal{T}_{\Sigma}$ that satisfies the following:

- $\left(\mathcal{V}_{\mathrm{ab}}^{\dagger}\right)^{-\frac{1}{2} c_{v}}$ is a functor from the category of marked surfaces to the category of line bundles over Teichmüller spaces of closed oriented surfaces.

- If we choose a symplectic basis of $H_{1}(\Sigma, \mathbb{Z})$ for a marked surface $\boldsymbol{\Sigma}$ then we get induced a connection in $\left(\mathcal{V}_{\mathrm{ab}}^{\dagger}\right)^{-\frac{1}{2} c_{v}}(L)$, whose curvature is $-\frac{1}{2} c_{v}$ times the curvature of the corresponding connection in $\mathcal{V}_{\mathrm{ab}}^{\dagger}$. The difference between the connections associated to two different bases is $-\frac{1}{2} c_{v}$ times the global scalar-value 1-form on $\mathcal{T}_{\Sigma}$ given in (68).

Proof. Let $\left(\left(\mathcal{V}_{\mathrm{ab}}^{\dagger}\right)^{\otimes 2}\right)^{*}$ be the complement of the zero section of $\left(\mathcal{V}_{\mathrm{ab}}^{\dagger}\right)^{\otimes 2}$. Let $\widetilde{\left(\mathcal{V}_{\mathrm{ab}}^{\dagger}\right)^{\otimes 2}}$ be the fiberwise universal cover of $\left(\left(\mathcal{V}_{\mathrm{ab}}^{\dagger}\right)^{\otimes 2}\right)^{*}$ based at the section $s(L)$. This is a completely functorial construction on pairs of line bundles and non-vanishing sections. There is a unique lift of the $\mathbb{C}^{*}$-action on $\left(\left(\mathcal{V}_{\mathrm{ab}}^{\dagger}\right)^{\otimes 2}\right)^{*}$ to a $\mathbb{C}$-action on $\left(\widehat{\mathcal{V}_{\mathrm{ab}}^{\dagger}}\right)^{\otimes 2}$ with respect to the covering map exp from $\mathbb{C}$ to $\mathbb{C}^{*}$. For any $\alpha \in \mathbb{C}^{*}$ we can now functorially define a line bundle $\left(\left(\mathcal{V}_{\mathrm{ab}}^{\dagger}\right)^{\otimes 2}\right)^{\alpha}(L)$ as follows:

$$
\left(\left(\mathcal{V}_{\mathrm{ab}}^{\dagger}\right)^{\otimes 2}\right)^{\alpha}(L)=\widetilde{\left(\mathcal{V}_{\mathrm{ab}}^{\dagger}\right)^{\otimes 2}} \times_{\rho_{\alpha}} \mathbb{C}
$$


where $\rho_{\alpha}(z): \mathbb{C} \rightarrow \mathbb{C}$ is the linear map given by multiplication by $\exp (\alpha z)$ for all $z \in \mathbb{C}$. We emphasis the dependence of this bundle on the section $s(L)$ and hence on $L$ in the notation for this bundle. Here we choose $\alpha=-c_{v} / 4$ to define $\left(\mathcal{V}_{\mathrm{ab}}^{\dagger}\right)^{-\frac{1}{2} c_{v}}$.

It is clear from the construction of $\left(\left(\mathcal{V}_{\mathrm{ab}}^{\dagger}\right)^{\otimes 2}\right)^{\alpha}(L)$, that a connection in $\mathcal{V}_{\mathrm{ab}}^{\dagger}$ will induce a connection in $\left(\left(\mathcal{V}_{\mathrm{ab}}^{\dagger}\right)^{\otimes 2}\right)^{\alpha}(L)$, whose curvature two-form is $\alpha / 2$ times the curvature two-form of that connection in $\mathcal{V}_{\mathrm{ab}}^{\dagger}$. For the construction of the functor on the morphisms of marked surfaces, we refer to [20] and [1].

By pulling $\left(\mathcal{V}_{\mathrm{ab}}^{\dagger}\right)^{-\frac{1}{2} c_{v}}(L)$ with its connection back to $\mathcal{T}_{(\Sigma, P)}$ from $\mathcal{T}_{\Sigma}$, we get a line bundle with with a connection on $\mathcal{T}_{(\Sigma, P)}$, which we also denote $\left(\mathcal{V}_{\mathrm{ab}}^{\dagger}\right)^{-\frac{1}{2} c_{v}}(L)$.

Let now $(\boldsymbol{\Sigma}, \lambda)=(\Sigma, P, V, L, \lambda)$ be a stable and saturated labeled marked surface. From the above Theorems 11.1 and 11.3. we see that there is a well defined flat connection in the vector bundle $\mathcal{V}_{\lambda}^{\dagger} \otimes\left(\mathcal{V}_{\mathrm{ab}}^{\dagger}\right)^{-\frac{1}{2} c_{v}}(L)$ over $\mathcal{T}_{(\Sigma, P)}$ gotten by taking the tensor product connection of the two connections induced by any symplectic basis $\left(\alpha_{i}, \beta_{i}\right)$ of $H_{1}(\Sigma, \mathbb{Z})$. Now $\mathcal{T}_{(\Sigma, P)}$ forms a $\mathbb{R}_{+}^{\mathrm{P}}$-principal bundle over the reduced Teichmüller space $\mathcal{T}^{(\mathrm{r})}{ }_{(\Sigma, P)}$. Hence we can use the flat connection to push forward this bundle to obtain a bundle with a flat connection over the reduced Teichmüller space.

Definition 11.1. For the stable and saturated labeled marked surface $(\boldsymbol{\Sigma}, \lambda)$ we define the vector bundle $\mathcal{V}_{\lambda}^{\dagger}(\boldsymbol{\Sigma})$ with its flat connection $\nabla(\boldsymbol{\Sigma}, \lambda)$ as the push forward of the bundle $\mathcal{V}_{\lambda}^{\dagger} \otimes\left(\mathcal{V}_{\mathrm{ab}}^{\dagger}\right)^{-\frac{1}{2} c_{v}}(L)$ to the reduced Teichmüller space $\mathcal{T}^{(\mathrm{r})}{ }_{(\Sigma, P)}$ followed by restriction to the fiber $\mathcal{T}_{\boldsymbol{\Sigma}}$.

Remark 11.3. By Theorem 11.1 and 11.2 and Lemma 6.3 we see that morphism of stable and saturated marked surfaces induces isomorphisms of flat vector bundles covering corresponding diffeomorphisms of Teichmüller spaces of the corresponding marked surfaces.

However, for a labeled marked surface $(\Sigma, \lambda)=(\Sigma, P, V, L, \lambda)$, which is not stable or not saturated we need to say a little more. Namely, let $\left(\boldsymbol{\Sigma}^{\prime}, \lambda^{\prime}\right)$ be obtained from $(\boldsymbol{\Sigma}, \lambda)$ by further labeling points not in $P$ by the zero label $0 \in P_{\ell}$ and choose projective tangent vectors at these new labeled points, such that $\boldsymbol{\Sigma}^{\prime}$ is both stable and saturated. . Let $\pi^{\prime}$ be the projection map from $\mathcal{T}_{\boldsymbol{\Sigma}^{\prime}}$ to $\mathcal{T}_{\boldsymbol{\Sigma}}$.

Proposition 11.1. The connection $\nabla\left(\boldsymbol{\Sigma}^{\prime}, \lambda^{\prime}\right)$ has trivial holonomy along the fibers of the projection map $\pi^{\prime}$. The connection $\nabla\left(\boldsymbol{\Sigma}^{\prime}, \lambda^{\prime}\right)$ induces a flat connection in the bundle over $\mathcal{T}_{\boldsymbol{\Sigma}}$ obtained by push forward $\mathcal{V}_{\lambda^{\prime}}^{\dagger}\left(\boldsymbol{\Sigma}^{\prime}\right)$ along $\pi^{\prime}$ using $\nabla\left(\boldsymbol{\Sigma}^{\prime}, \lambda^{\prime}\right)$. If $\left(\boldsymbol{\Sigma}^{\prime \prime}, \lambda^{\prime \prime}\right)$ is another stable and saturated labeled marked surface obtained from $(\boldsymbol{\Sigma}, \lambda)$ in the same way by adding zero-labeled point to $P$, then iterations of the propagation of vacua isomorphisms given in Proposition 5.3 induces a connection preserving isomorphism between the corresponding pair of bundles over $\mathcal{T}_{\boldsymbol{\Sigma}}$.

This proposition follows directly from Proposition 6.7 and the definition of the flat vector bundle $\mathcal{V}_{\lambda^{\prime}}^{\dagger}\left(\boldsymbol{\Sigma}^{\prime}, P^{\prime}\right)$. 
Definition 11.2. We define the vector bundle with its flat connection $\left(\mathcal{V}_{\lambda}^{\dagger}(\boldsymbol{\Sigma}), \nabla(\boldsymbol{\Sigma}, \lambda)\right)$ over $\mathcal{T}_{\boldsymbol{\Sigma}}$ to be $\pi_{*}^{\prime}\left(\mathcal{V}_{\lambda^{\prime}}^{\dagger}\left(\boldsymbol{\Sigma}^{\prime}\right), \nabla\left(\boldsymbol{\Sigma}^{\prime}, \lambda^{\prime}\right)\right)$ for any stable and saturated labeled marked surface $\left(\boldsymbol{\Sigma}^{\prime}, \lambda^{\prime}\right)$ obtained from $(\boldsymbol{\Sigma}, \lambda)$ by adding zero-labeled marked points to $P$.

Suppose now $f:\left(\boldsymbol{\Sigma}_{1}, \lambda_{1}\right) \rightarrow\left(\boldsymbol{\Sigma}_{2}, \lambda_{2}\right)$ is a morphism of labeled marked surfaces and that $\left(\boldsymbol{\Sigma}_{i}^{\prime}, \lambda_{i}^{\prime}\right)$ is obtained as above from $\left(\boldsymbol{\Sigma}_{i}, \lambda_{i}\right)$ by adding zero-labeled points and further that $f^{\prime}:\left(\boldsymbol{\Sigma}_{1}^{\prime}, \lambda_{1}^{\prime}\right) \rightarrow\left(\boldsymbol{\Sigma}_{2}^{\prime}, \lambda_{2}^{\prime}\right)$ is any morphism of labeled marked surfaces, which induces $f$ when restricted to $\left(\boldsymbol{\Sigma}_{1}, \lambda_{1}\right)$. We then have the following result as a direct consequence of Proposition 6.8 .

Proposition 11.2. The induced morphism of flat vector bundles $\mathcal{V}^{\dagger}\left(f^{\prime}\right): \mathcal{V}_{\lambda_{1}^{\prime}}^{\dagger}\left(\boldsymbol{\Sigma}_{1}^{\prime}\right) \rightarrow \mathcal{V}_{\lambda_{2}^{\prime}}^{\dagger}\left(\boldsymbol{\Sigma}_{2}^{\prime}\right)$ induces a morphism of flat vector bundles from $\mathcal{V}_{\lambda_{1}}^{\dagger}\left(\boldsymbol{\Sigma}_{1}\right)$ to $\mathcal{V}_{\lambda_{2}}^{\dagger}\left(\boldsymbol{\Sigma}_{2}\right)$ which only depends on $f$ and which behaves well under compositions of morphism of labeled marked surface.

Let us now collect the thus fare obtained in the following theorem.

Theorem 11.4. The construction given above gives a functor from the category of labeled marked surfaces to the category of vector bundles with flat connections over Teichmüller spaces of marked surfaces.

The modular functor we seek is now simply just obtained by composing with the functor which takes covariant constant sections of vector bundles with connections.

Definition 11.3 (The functor $V_{\ell}^{\mathfrak{g}}$ ). Let $\ell$ be a positive integer. Let $P_{\ell}$ be the finite set defined in (6) with the involution $\dagger$ as defined by (3). Let $(\boldsymbol{\Sigma}, \lambda)=(\Sigma, P, V, L, \lambda)$ be a labeled marked surface using the label set $P_{\ell}$. The functor $V_{\ell}^{\mathfrak{g}}$ is by definition the composite of the functor, which assigns to $(\boldsymbol{\Sigma}, \lambda)$ the flat vector bundle $\mathcal{V}_{\lambda}^{\dagger}(\boldsymbol{\Sigma})$ over $\mathcal{T}_{\boldsymbol{\Sigma}}$, and the functor, which takes covariant constant sections.

Remark 11.4. For a labeled marked surface $(\boldsymbol{\Sigma}, \lambda)$ and a complex structure $\mathbf{C}$ on it, we see that Proposition 5.2 and 8.2 give an isomorphism

$$
V_{\ell}^{\mathfrak{g}}(\boldsymbol{\Sigma}, \lambda) \cong \mathcal{V}_{\lambda}^{\dagger}(\mathbf{C}) \otimes\left(\mathcal{V}_{\mathrm{ab}}^{\dagger}\right)^{-\frac{1}{2} c_{v}}(L)(\mathbf{C})
$$

since $\mathcal{T}_{\boldsymbol{\Sigma}}$ is contractible. Moreover, if $\mathbf{f}:\left(\boldsymbol{\Sigma}_{1}, \lambda_{1}\right) \rightarrow\left(\boldsymbol{\Sigma}_{2}, \lambda_{2}\right)$ is a morphism of labeled marked Riemann surfaces, which is realized by a morphism of labeled marked Riemann surfaces $\Phi: \mathbf{C}_{1} \rightarrow \mathbf{C}_{2}$, such that $\Phi^{*}\left(L_{2}\right)=L_{1}$, then we have the following commutative diagram

$$
\begin{aligned}
& V_{\ell}^{\mathfrak{g}}\left(\boldsymbol{\Sigma}_{1}, \lambda_{1}\right) \stackrel{\cong}{\longrightarrow} \mathcal{V}_{\lambda}^{\dagger}\left(\mathbf{C}_{1}\right) \otimes\left(\mathcal{V}_{\mathrm{ab}}^{\dagger}\right)^{-\frac{1}{2} c_{v}}\left(L_{1}\right)\left(\mathbf{C}_{1}\right) \\
& V_{\ell}^{\mathfrak{g}}(\mathbf{f}) \downarrow \\
& V_{\ell}^{\mathfrak{g}}\left(\boldsymbol{\Sigma}_{2}, \lambda_{2}\right) \stackrel{\cong}{\longrightarrow} \mathcal{V}_{\lambda}^{\dagger}\left(\mathbf{C}_{2}\right) \otimes\left(\mathcal{V}_{\mathrm{ab}}^{\dagger}(\Phi)^{-\frac{1}{2} c_{v}} \downarrow\right. \\
&
\end{aligned}
$$


Remark 11.5. Let $(\boldsymbol{\Sigma}, \lambda)$ be a labeled marked surface and suppose that $\left(\boldsymbol{\Sigma}^{\prime}, \lambda^{\prime}\right)$ is obtained from $(\boldsymbol{\Sigma}, \lambda)$ by labeling further points by $0 \in P_{\ell}$, then by Proposition 11.1 we get induced an isomorphism

$$
V_{\ell}^{\mathfrak{g}}(\boldsymbol{\Sigma}, \lambda) \cong V_{\ell}^{\mathfrak{g}}\left(\boldsymbol{\Sigma}^{\prime}, \lambda^{\prime}\right) .
$$

Let $(\boldsymbol{\Sigma}, \lambda)$ be a labeled marked surface. Let $\mathfrak{F}=(\pi: \mathcal{C} \rightarrow \mathcal{B}, \vec{s}, \vec{\eta})$ be a family of stable and saturated Riemann surfaces with formal neighbourhoods over $\boldsymbol{\Sigma}$.

Definition 11.4. We define $V_{\ell}^{\mathfrak{g}}(\mathfrak{F}, \lambda)$ to be the covariant constant sections of the flat bundles $\mathcal{V}_{\lambda}^{\dagger}(\mathfrak{F}) \otimes\left(\mathcal{V}_{\mathrm{ab}}^{\dagger}\right)^{-\frac{1}{2} c_{v}}(L)(\mathfrak{F})$ over $\mathcal{B}$.

Form this definition it is clear that we get an isomorphism

$$
I_{\mathfrak{F}}: V_{\ell}^{\mathfrak{g}}(\mathfrak{F}, \lambda) \rightarrow V_{\ell}^{\mathfrak{g}}(\boldsymbol{\Sigma}, \lambda) .
$$

In order for the functor $V_{\ell}^{\mathfrak{g}}$ to be modular, we need to further construct the disjoint union isomorphism and the glueing isomorphism and to check that the axioms of a modular functor is satisfied. First we construct the disjoint union isomorphism. The glueing isomorphism will be constructed in the following section.

Let $\left(\boldsymbol{\Sigma}_{i}, \lambda_{i}\right)=\left(\Sigma_{i}, P_{i}, V_{i}, L_{i}, \lambda_{i}\right), i=1,2$, be two stable and saturated labeled marked surfaces and let $(\boldsymbol{\Sigma}, \lambda)=\left(\boldsymbol{\Sigma}_{1}, \lambda_{1}\right) \sqcup\left(\boldsymbol{\Sigma}_{2}, \lambda_{2}\right)$. Let $\lambda=\lambda_{1} \sqcup \lambda_{2}$. We have that $\mathcal{T}_{\boldsymbol{\Sigma}}=\mathcal{T}_{\boldsymbol{\Sigma}_{1}} \times \mathcal{T}_{\boldsymbol{\Sigma}_{2}}$. Let $\pi_{i}: \mathcal{T}_{\boldsymbol{\Sigma}} \rightarrow \mathcal{T}_{\boldsymbol{\Sigma}_{i}}$ be the projection onto the $i$ 'th factor. We clearly have that

Proposition 11.3. The natural isomorphism $\mathcal{H}_{\vec{\lambda}} \cong \mathcal{H}_{\vec{\lambda}_{1}} \otimes \mathcal{H}_{\vec{\lambda}_{2}}$ (for any ordering $\vec{\lambda}, \vec{\lambda}_{1}$ and $\vec{\lambda}_{2}$ of $\lambda, \lambda_{1}$ and $\lambda_{2}$ respectively) induces an isomorphism of vector bundles with connections

$$
\mathcal{V}_{\lambda}^{\dagger}(\Sigma, P) \cong \pi_{1}^{*} \mathcal{V}_{\lambda_{1}}^{\dagger}\left(\Sigma_{1}, P_{1}\right) \otimes \pi_{2}^{*} \mathcal{V}_{\lambda_{2}}^{\dagger}\left(\Sigma_{2}, P_{2}\right)
$$

where we use the Lagrangian subspaces to fix the connections in all three bundles. The isomorphism is compatible with isomorphism induced by disjoint union of morphism of corresponding labeled marked surfaces.

Remark 11.6. These disjoint union isomorphisms are clearly compatible with the propagation of vacua isomorphisms given in Proposition 11.1.

Further it is easy to see that

Proposition 11.4. The isomorphism given in Lemma 10.1 induces an isomorphism of line bundles with connections

$$
\mathcal{V}_{\mathrm{ab}}^{\dagger}(\Sigma) \cong \pi_{1}^{*} \mathcal{V}_{\mathrm{ab}}^{\dagger}\left(\Sigma_{1}\right) \otimes \pi_{2}^{*} \mathcal{V}_{\mathrm{ab}}^{\dagger}\left(\Sigma_{2}\right)
$$

where we use the Lagrangian subspaces to fix the connections in all three bundles. The isomorphism is compatible with isomorphism induced by disjoint union of morphism of corresponding labeled marked surfaces. Moreover the preferred sections of the squares of these bundles specified by the given Lagrangian subspaces are compatible with this isomorphism. 
From this proposition it then follows that we get the corresponding isomorphism of $-\frac{1}{2} c_{v}$-power of these bundles. Combining this with (73) we now get induced a preferred isomorphism of flat vector bundles

$$
\mathcal{V}_{\lambda}^{\dagger}(\boldsymbol{\Sigma}) \cong \pi_{1}^{*} \mathcal{V}_{\lambda_{1}}^{\dagger}\left(\boldsymbol{\Sigma}_{1}\right) \otimes \pi_{2}^{*} \mathcal{V}_{\lambda_{2}}^{\dagger}\left(\boldsymbol{\Sigma}_{2}\right)
$$

which intern induces the required isomorphism of the corresponding vector spaces of covariant constant sections:

$$
V_{\ell}^{\mathfrak{g}}(\boldsymbol{\Sigma}, \lambda) \cong V_{\ell}^{\mathfrak{g}}\left(\boldsymbol{\Sigma}_{1}, \lambda_{1}\right) \otimes V_{\ell}^{\mathfrak{g}}\left(\boldsymbol{\Sigma}_{2}, \lambda_{2}\right)
$$

which is natural with respect to disjoint union of morphisms.

\section{Sheaf of VACUA AND GLUiNG.}

Let $\boldsymbol{\Sigma}=\left(\Sigma,\left\{p_{-}, p_{+}\right\} \sqcup P,\left\{v_{-}, v_{+}\right\} \sqcup V, L\right)$ be a marked surface. Let

$$
c: P\left(T_{p_{-}} \Sigma\right) \rightarrow P\left(T_{p_{+}} \Sigma\right)
$$

be a glueing map and $\Sigma_{c}$ the glueing of $\Sigma$ at the ordered pair $\left(\left(p_{-}, v_{-}\right),\left(p_{+}, v_{+}\right)\right)$with respect to $c$ as described in section 2, We shall first assume that $\left(\Sigma_{c}, P\right)$ is stable and saturated.

Let $\mathfrak{F}=\left(\pi: \mathcal{C} \rightarrow \mathcal{B} ; s_{-}, s_{+}, \vec{s} ; \eta_{-}, \eta_{+}, \vec{\eta}\right)$ be a family of pointed Riemann surfaces with formal neighbourhoods on $\boldsymbol{\Sigma}$ over a simply-connected base $\mathcal{B}$. Let $D$ be the unit disk in the complex plane. Assume we have holomorphic functions $x_{ \pm}: U_{ \pm} \subset \mathcal{C} \rightarrow D$ such that for each $b \in \mathcal{B}$ we have that $\left.x_{ \pm}\right|_{\pi^{-1}(b)}: U_{ \pm} \cap \pi^{-1}(b) \rightarrow D$ are local coordinates for $\pi^{-1}(b)$ centered at $p_{ \pm}$and further that $x_{ \pm}=\eta_{ \pm}$ as formal neighbourhoods. Further we assume that $d_{p_{ \pm}}\left(\left.x_{ \pm}\right|_{\pi^{-1}(b)}\right)\left(v_{ \pm}\right)=1$ and that

$$
c=P\left(d_{p_{+}}\left(\left.x_{+}\right|_{\pi^{-1}(b)}\right)\right)^{-1} \circ P(\bar{*}) \circ P\left(d_{p_{-}}\left(\left.x_{-}\right|_{\pi^{-1}(b)}\right)\right): P\left(T_{p_{-}} \Sigma\right) \rightarrow P\left(T_{p_{+}} \Sigma\right)
$$

where $P(\bar{*}): P\left(T_{0} D\right) \rightarrow P\left(T_{0} D\right)$ is induced by the the real linear map $z \mapsto \bar{z}$. Assume that $P \subset$ $\Sigma-\left(U_{-} \cup U_{+}\right)$.

Set $\mathcal{B}_{c}=\mathcal{B} \times D$ and $\pi_{D}: \mathcal{B}_{c} \rightarrow D$ be the projection onto the second factor. Let us now construct a stable and saturated family of pointed curves with formal neighbourhoods $\mathfrak{F}_{c}=\left(\pi_{c}: \mathcal{C}_{c} \rightarrow \mathcal{B}_{c}, \vec{s}, \vec{\eta}\right)$, in the sense of Definition 14.2 in the Appendix below, by applying the glueing construction pointwise over $\mathcal{B}$ to $\mathfrak{F}$ :

Let

$$
\begin{gathered}
\mathcal{C}^{1}=\left\{(z, w, \tau) \in D^{\times 3} \mid z w=\tau\right\} \\
\mathcal{C}_{c}^{1}=C^{1} \times \mathcal{B}
\end{gathered}
$$

and

$$
\mathcal{C}_{c}^{2}=\left\{(y, \tau) \in \mathcal{C} \times D\left|y \in U_{ \pm} \Rightarrow\right| x_{ \pm}(y)|>| \tau \mid\right\}
$$

Let then

$$
\mathcal{C}_{c}=\mathcal{C}_{c}^{1} \cup_{\phi} \mathcal{C}_{c}^{2}
$$

where

$$
\phi:\left(\left(U_{-}-p_{-}\right) \times D \cup\left(U_{+}-p_{+}\right) \times D\right) \cap \mathcal{C}_{c}^{2} \rightarrow \mathcal{C}_{c}^{1}
$$


is given by

$$
\phi(y, \tau)=\left\{\begin{array}{ll}
\left(x_{-}(y), \tau / x_{-}(y), \tau, \pi(y)\right), & y \in U_{-}-p_{-} \\
\left(\tau / x_{+}(y), x_{+}(y), \tau, \pi(y)\right), & y \in U_{+}-p_{+}
\end{array} .\right.
$$

One easily checks that $\mathcal{C}_{c}$ is a smooth complex manifold of $\operatorname{dimension} \operatorname{dim}(\mathcal{B})+2$ and that we have an obvious holomorphic projection map $\pi_{c}: \mathcal{C}_{c} \rightarrow \mathcal{B}_{c}$. Let $\mathfrak{F}_{c}=\left(\pi_{c}: \mathcal{C}_{c} \rightarrow \mathcal{B}_{c}, \vec{s}, \vec{\eta}\right)$.

The fibers over $\mathcal{B} \times\{0\}$ are nodal curves, hence $\mathfrak{F}_{c}$ is not a family of pointed Riemann surfaces with formal neighbourhoods, however it is a family of pointed stable curves with formal neighbourhoods in the sense of Definition 14.2 in the Appendix below. If $D^{*}=D \backslash\{0\}$, then the restricted family $\left.\mathfrak{F}_{c}\right|_{\mathcal{B} \times D^{*}}$ is however a family of pointed Riemann surfaces with formal neighbourhoods.

Set $\tilde{D}=\{\zeta \in \mathbb{C} \mid \operatorname{Im}(\zeta)>0\}$. On $\tilde{D}$ we now consider the real coordinates $(r, \theta)$ given by $r(\zeta)=\exp (-2 \pi \operatorname{Im}(\zeta))$ and $\theta(\zeta)=\operatorname{Re}(\zeta)$. Let $\left(r_{ \pm}, \theta_{ \pm}\right)$be $x_{ \pm}$composed with polar coordinates.

Let $\tilde{\mathcal{B}}_{c}=\mathcal{B} \times \tilde{D}$ and $p_{c}: \tilde{\mathcal{B}}_{c} \rightarrow \mathcal{B}_{c}^{*}=\mathcal{B} \times D^{*}$ be given by $p_{c}(b, \zeta)=(b, \exp (2 \pi i \zeta))$. Then $\tilde{\mathcal{B}}_{c}$ is the universal cover of $\mathcal{B}_{c}$. Let $\tilde{\mathcal{C}}_{c}=p_{c}^{*} \mathcal{C}_{c}, \mathcal{C}_{c}^{\prime}=\left.\mathcal{C}_{c}\right|_{\mathcal{B}_{c}^{*}}, \tilde{\mathfrak{F}}_{c}=p_{c}^{*} \mathfrak{F}_{c}, \tilde{\pi}_{c}: \tilde{\mathcal{C}}_{c} \rightarrow \tilde{\mathcal{B}}_{c}, \tilde{\pi}_{\tilde{D}}: \tilde{\mathcal{B}}_{c} \rightarrow \tilde{D}$ and $\tilde{\pi}_{\mathcal{B}}: \tilde{\mathcal{B}}_{c} \rightarrow \mathcal{B}$.

Let

$$
V_{ \pm}=\Phi_{\mathfrak{F}}^{-1}\left(U_{ \pm}\right)
$$

and

$$
\tilde{x}_{ \pm}: V_{ \pm} \rightarrow \mathcal{B} \times D
$$

be given by

$$
\tilde{x}_{ \pm}=\left(\pi_{\mathcal{B}}, x_{ \pm} \circ \Phi_{\mathfrak{F}}\right) .
$$

Let us now define a fiber preserving diffeomorphism

$$
f: \tilde{\mathcal{C}}_{c} \rightarrow \Sigma_{c} \times \tilde{B}_{c}
$$

by

$$
f(y, r, \theta)= \begin{cases}\left(\tilde{x}_{-}^{-1}\left(\pi(y), \chi_{r}\left(r_{-}(y)\right), \theta_{-}(y)+\frac{1}{2} \frac{1-r_{-}(y)}{1-r^{1 / 2}} \theta\right), r, \theta\right) & \text { if } y \in U_{-}, 1 \geq r_{-}(y) \geq r^{1 / 2} \\ \left(\tilde{x}_{+}^{-1}\left(\pi(y),-\chi_{r}\left(r_{-}(y)\right),-\theta_{-}(y)-\frac{1}{2} \frac{r_{-}(y)-r}{r^{1 / 2}-r} \theta\right), r, \theta\right) & \text { if } y \in U_{-}, r^{1 / 2} \geq r_{-}(y) \geq r\end{cases}
$$

and extend $f$ to all of $\tilde{\mathcal{C}}_{c}$ by the map $\Phi_{\mathfrak{F}}^{-1} \times \operatorname{Id}$ on $\left(\mathcal{C}-\left(U_{+} \cup U_{-}\right)\right) \times \tilde{D}$.

Here $\chi_{r}$ is a smooth family of diffeomorphisms

$$
\chi_{r}:[1, r] \rightarrow[1,-1], r \in(0,1)
$$

with the properties that $\chi_{r}$ is the identity near $1, \chi_{r}$ maps $\rho \mapsto-r / \rho$ near $r$ and $\chi_{r}\left(r^{1 / 2}\right)=0$ for each $r \in(0,1)$. We will furthermore assume that for all $\rho \in(0,1)$ we have that

$$
\lim _{r \rightarrow 0} \chi_{r}(\rho)=\rho \text { and } \lim _{r \rightarrow 0} \chi_{r}(r / \rho)=-\rho
$$

for all $\rho \in(0,1)$. 
The extra conditions on $\chi_{r}$ implies that the $\operatorname{limit}_{\lim _{r} \rightarrow 0} q \circ f(\cdot, r, 0): \Sigma^{\prime} \rightarrow \Sigma^{\prime}$ exists and is equal to Id : $\Sigma^{\prime} \rightarrow \Sigma^{\prime}$. We observe that the monodromy $\left(\left.f\right|_{\{b\} \times \pi^{-1}(\zeta+1)}\right) \circ\left(\left.f\right|_{\{b\} \times \pi^{-1}(\zeta)}\right)^{-1}$ is a Dehn twist in $P\left(T_{p_{-}} \Sigma\right)$.

Using $f^{-1}: \Sigma_{c} \times \tilde{\mathcal{B}}_{c} \rightarrow \tilde{\mathcal{C}}_{c}$ we see that $\tilde{\mathfrak{F}}_{c}$ is a family of stable and saturated curves with formal neighbourhoods on $\Sigma_{c}$.

The inclusion

$$
\mathcal{H}_{\vec{\lambda}}(\mathcal{B}) \hookrightarrow \bigoplus_{\mu \in P_{\ell}} \mathcal{H}_{\mu, \mu^{\dagger}, \vec{\lambda}}(\mathcal{B})
$$

given by

$$
|\phi\rangle \mapsto \bigoplus_{\mu \in P_{\ell}}\left|0_{\mu, \mu^{\dagger}} \otimes \phi\right\rangle
$$

induces an isomorphism of vector bundles

$$
\mathcal{V}_{\vec{\lambda}}^{\dagger}\left(\left.\mathfrak{F}_{c}\right|_{\mathcal{B} \times\{0\}}\right) \cong \bigoplus_{\mu \in P_{\ell}} \mathcal{V}_{\mu, \mu^{\dagger}, \vec{\lambda}}^{\dagger}(\mathfrak{F})
$$

This is the content of Theorem 4.4.9 in [19.

The abelian sheaf of vacua construction applied to the family $\mathfrak{F}_{c}$ gives a holomorphic line bundle $\mathcal{V}_{\mathrm{ab}}^{\dagger}\left(\mathfrak{F}_{c}\right)$ over $\mathcal{B}_{c}$. This follows from Theorem 14.2 below. We get an isomorphism of vector bundles

$$
\left.\mathcal{V}_{\mathrm{ab}}^{\dagger}\left(\mathfrak{F}_{c}\right)\right|_{\mathcal{B} \times\{0\}} \cong \mathcal{V}_{\mathrm{ab}}^{\dagger}(\mathfrak{F})
$$

induced by the isomorphism given in Theorem 14.3 below. The preferred section $s_{\mathfrak{F}_{c}}\left(L_{c}\right)$ is continuous over $\mathcal{B}_{c}$. Over $\pi_{D}^{-1}(0)$ it is mapped via the above isomorphism to the preferred section $s_{\mathfrak{F}}(L)$ of $\mathcal{V}_{\mathrm{ab}}^{\dagger}(\mathfrak{F})$. This follows by Theorem 14.4 and 14.8. As discussed before the Lagrangian subspace $L$ determines connections in the bundles $\oplus_{\mu} \mathcal{V}_{\mu, \mu^{\dagger}, \vec{\lambda}}^{\dagger}(\mathfrak{F})$ and $\mathcal{V}_{\mathrm{ab}}^{\dagger}(\mathfrak{F})$.

Proposition 12.1. The Lagrangian subspace $L_{c} \subset H_{1}\left(\Sigma_{c}, \mathbb{Z}\right)$ determines a unique normalized symmetric bidifferential $\omega_{c} \in H^{0}\left(\mathcal{C}_{c} \times{ }_{\mathcal{B}_{c}} \mathcal{C}_{c}, \omega_{\mathcal{C}_{c} \times \mathcal{B}_{c} \mathcal{C}_{c}}(2 \Delta)\right)$ specified by formula (42) for any symplectic basis $(\vec{\alpha}, \vec{\beta})$ of $H_{1}\left(\Sigma_{c}, \mathbb{Z}\right)$ such that $L_{c}=\operatorname{Span}\left\{\beta_{i}\right\}$.

Proof. Given any symplectic basis $(\vec{\alpha}, \vec{\beta})$ of $H_{1}\left(\Sigma_{c}, \mathbb{Z}\right)$ such that $L_{c}=\operatorname{Span}\left\{\beta_{i}\right\}$, formula (42) defines a normalized symmetric bidifferential $\tilde{\omega}_{c} \in H^{0}\left(\tilde{\mathcal{C}}_{c} \times_{\tilde{\mathcal{B}}_{c}} \tilde{\mathcal{C}}_{c}, \omega_{\tilde{\mathcal{C}}_{c}} \times_{\tilde{\mathcal{B}}_{c}} \tilde{\mathcal{C}}_{c}(2 \Delta)\right)$. The monodromy of the fibration $\pi:\left.\mathcal{C}_{c}\right|_{\tilde{\pi}_{\mathcal{B}}^{-1}(b)} \rightarrow D$ is the Dehn twist in the Riemann surface $P\left(T_{p_{-}} \Sigma\right)$, hence it preserves $L_{c}$. But then by applying the transformation law given in (5.2.7) and (5.2.8) [19], we see that $\tilde{\omega}_{c}$ is invariant under this monodromy and therefore $\tilde{\omega}_{c}$ is the pull back of a unique

$$
\omega_{c}^{\prime} \in H^{0}\left(\mathcal{C}_{c}^{\prime} \times{ }_{\mathcal{B}_{c}} \mathcal{C}_{c}^{\prime}, \omega_{\mathcal{C}_{c}^{\prime} \times{ }_{\mathcal{B}}} \mathcal{C}_{c}^{\prime}(2 \Delta)\right)
$$

By the general theory for bidifferentials on such families, see e.g. 9] chapter III, we have that there is a unique $\omega_{c} \in H^{0}\left(\mathcal{C}_{c} \times \mathcal{B}_{c} \mathcal{C}_{c}, \omega_{\mathcal{C}_{c} \times \mathcal{B}_{c}} \mathcal{C}_{c}(2 \Delta)\right)$ such that $\left.\omega_{c}\right|_{\mathcal{C}_{c}^{\prime} \times \mathcal{B}_{c}^{*} \mathcal{C}_{c}^{\prime}}=\omega_{c}^{\prime}$. 
By Definition 6.2 and Theorem 6.2 we get that $\omega_{c}$ determines a projectively flat connection in $\left.\mathcal{V}_{\vec{\lambda}}^{\dagger}\left(\mathfrak{F}_{c}\right)\right|_{\mathcal{B}_{c}^{*}}$ and by Theorem 9.1 a connection in $\left.\mathcal{V}_{\mathrm{ab}}^{\dagger}\left(\mathfrak{F}_{c}\right)\right|_{\mathcal{B}_{c}^{*}}$.

Let us now recall the conclusion of the glueing constructions on the sheaf of vacua both in the non-abelian and abelian case applied to the family $\mathfrak{F}_{c}$ :

The explicit formula (80) and Theorem 14.6 below give an isomorphism between sections of $\left.\mathcal{V}_{\vec{\lambda}}^{\dagger}\left(\mathfrak{F}_{c}\right)\right|_{\mathcal{B} \times\{0\}}$ and sections of $\mathcal{V}_{\vec{\lambda}}^{\dagger}\left(\tilde{\mathfrak{F}}_{c}\right)$, which are covariant constant along the fibers of $\tilde{\pi}_{\tilde{D}}$.

The connection in $\left.\mathcal{V}_{\mathrm{ab}}^{\dagger}\left(\mathfrak{F}_{c}\right)\right|_{\mathcal{B}_{c}^{*}}$ determined by $\omega_{c}$ extends to a connection on all of $\mathcal{V}_{\mathrm{ab}}^{\dagger}\left(\mathfrak{F}_{c}\right)$, hence we get by parallel transport along the fibers of $\pi_{D}$ an isomorphism between sections of $\left.\mathcal{V}_{\text {ab }}^{\dagger}\left(\mathfrak{F}_{c}\right)\right|_{\mathcal{B} \times\{0\}}$ and sections of $\mathcal{V}_{\mathrm{ab}}^{\dagger}\left(\mathfrak{F}_{c}\right)$, which are covariant constant along the fibers of $\pi_{D}$. This follows from Theorem 14.7 below and formula (82) gives an explicit formula for this isomorphism.

By applying the fractional power construction to the line bundle $\mathcal{V}_{\mathrm{ab}}^{\dagger}\left(\mathfrak{F}_{c}\right)^{\otimes 2}$ with the preferred section $s_{\mathfrak{F}_{c}}\left(L_{c}\right)$, we get a line bundle over $\mathcal{B}_{c}$, which we denote $\mathcal{V}_{\mathrm{ab}}^{\dagger}\left(\mathfrak{F}_{c}\right)^{-\frac{1}{2} c_{v}}\left(L_{c}\right)$. By the very construction of this bundle we see that $\left.\mathcal{V}_{\mathrm{ab}}^{\dagger}\left(\mathfrak{F}_{c}\right)^{-\frac{1}{2} c_{v}}\left(L_{c}\right)\right|_{\mathcal{B} \times\{0\}}$ is identified with $\left(\mathcal{V}_{\mathrm{ab}}^{\dagger}\right)(\mathfrak{F})^{-\frac{1}{2} c_{v}}(L)$. We get a connection in this bundle from its construction and an isomorphism from sections of $\left(\mathcal{V}_{\mathrm{ab}}^{\dagger}\right)(\mathfrak{F})^{-\frac{1}{2} c_{v}}(L)$ to sections of $\mathcal{V}_{\mathrm{ab}}^{\dagger}\left(\mathfrak{F}_{c}\right)^{-\frac{1}{2} c_{v}}\left(L_{c}\right)$ over $\mathcal{B}_{c}$, which are covariant constant along the fibers of $\pi_{D}$.

Theorem 12.1. The tensor product of these two glueing constructions gives an isomorphism $I_{c}\left(\mathfrak{F}, x_{ \pm}\right)$ from covariant constant sections of $\bigoplus_{\mu \in P_{\ell}} \mathcal{V}_{\mu, \mu^{\dagger}, \vec{\lambda}}^{\dagger}(\mathfrak{F}) \otimes \mathcal{V}_{\mathrm{ab}}^{\dagger}(\mathfrak{F})^{-\frac{1}{2} c_{v}}(L)$ over $\mathcal{B}$ to covariant constant sections of $\mathcal{V}_{\vec{\lambda}}^{\dagger}\left(\tilde{\mathfrak{F}}_{c}\right) \otimes \mathcal{V}_{\mathrm{ab}}^{\dagger}\left(\tilde{\mathfrak{F}}_{c}\right)^{-\frac{1}{2} c_{v}}\left(L_{c}\right)$ over $\tilde{\mathcal{B}}_{c}$ :

$$
I_{c}\left(\mathfrak{F}, x_{ \pm}\right): \bigoplus_{\mu \in P_{\ell}} V_{\ell}^{\mathfrak{g}}\left(\mathfrak{F}, \mu, \mu^{\dagger}, \lambda\right) \rightarrow V_{\ell}^{\mathfrak{g}}\left(\tilde{\mathfrak{F}}_{c}, \lambda\right) .
$$

Proof. From an element of $\bigoplus_{\mu \in P_{\ell}} V_{\ell}^{\mathfrak{g}}\left(\mathfrak{F}, \mu, \mu^{\dagger}, \lambda\right)$, we get a section of $\left.\mathcal{V}_{\vec{\lambda}}^{\dagger}\left(\mathfrak{F}_{c}\right) \otimes \mathcal{V}_{\mathrm{ab}}^{\dagger}\left(\mathfrak{F}_{c}\right)^{-\frac{1}{2} c_{v}}\left(L_{c}\right)\right|_{\mathcal{B} \times\{0\}}$. By Theorem 5.3 and Remark 5.1 in 2] we see that the covariant derivative of the section of $\mathcal{V}_{\vec{\lambda}}^{\dagger}\left(\tilde{\mathfrak{F}}_{c}\right) \otimes \mathcal{V}_{\text {ab }}^{\dagger}\left(\tilde{\mathfrak{F}}_{c}\right)^{-\frac{1}{2} c_{v}}\left(L_{c}\right)$ obtained by glueing vanishes, since the contributions from the non-abelian factor exactly cancels the contribution form the $-\frac{1}{2} c_{v}$-power of the abelian factor. Hence glueing results in a covariant constant section of $\mathcal{V}_{\vec{\lambda}}^{\dagger}\left(\tilde{\mathfrak{F}}_{c}\right) \otimes \mathcal{V}_{\mathrm{ab}}^{\dagger}\left(\tilde{\mathfrak{F}}_{c}\right)^{-\frac{1}{2} c_{v}}\left(L_{c}\right)$ over $\tilde{\mathcal{B}}_{c}$. Since the two glueing constructions give isomorphisms, it is clear that $I_{c}$ is an isomorphism.

Let $\mathbf{C}^{(i)}, i=1,2$, be two complex structures on $\Sigma$ and let $x_{ \pm}^{(i)}: U_{ \pm}^{(i)} \rightarrow D$ be coordinates around $p_{ \pm}$with $d_{p_{ \pm}} x_{ \pm}^{(i)}\left(v_{ \pm}\right)=1$ such that $c=P\left(\left(d_{p_{+}} x_{+}^{(i)}\right)^{-1} \circ P(\bar{*}) \circ P\left(d_{p_{-}} x_{-}^{(i)}\right): P\left(T_{p_{-}} \Sigma\right) \rightarrow P\left(T_{p_{+}} \Sigma\right)\right.$. Let $\eta_{j}^{(i)}$ be formal coordinates around $p_{j} \in C^{(i)}$.

Theorem 12.2. For such two pairs $\left(\mathbf{C}^{(i)}, x_{ \pm}^{(i)}\right), i=1,2$, of complex structures and holomorphic coordinates on $\left(\Sigma,\left\{p_{+}, p_{-}\right\} \cup P\right)$ we have that

$$
I_{c}\left(\mathbf{C}^{(1)}, x_{ \pm}^{(1)}\right)=I_{c}\left(\mathbf{C}^{(2)}, x_{ \pm}^{(2)}\right) .
$$


This follows straight from Theorem 12.1, since we clearly have the following

Lemma 12.1. There exists a family of pointed Riemann surfaces with formal neighbourhoods $\mathfrak{F}=$ $(\pi: \mathcal{C} \rightarrow \mathcal{B}, \vec{s}, \vec{\eta})$ on $(\Sigma, P)$, holomorphic functions $x_{ \pm}: U_{ \pm} \subset \mathcal{C} \rightarrow D$ and $b_{i} \in \mathcal{B} i=1,2$ such that the following holds

- The base $\mathcal{B}$ is simply-connected.

- Restriction to the fiber

$$
\left(\pi^{-1}\left(b_{i}\right),\left.\vec{\eta}\right|_{\pi^{-1}\left(b_{i}\right)},\left.x_{ \pm}\right|_{\pi^{-1}\left(b_{i}\right)}: U_{ \pm} \cap \pi^{-1}\left(b_{i}\right) \rightarrow D\right)
$$

over $b_{i}, i=1,2$, is the same complex structure on $(\Sigma, P)$ as

$$
\left(\mathbf{C}^{(i)}, \vec{\eta}^{(i)}, x_{ \pm}^{(i)}: U_{ \pm}^{(i)} \rightarrow D\right)
$$

with the same formal coordinates and the same coordinates around $p_{ \pm}$.

- For each $b \in \mathcal{B}$ we have that $\left.x_{ \pm}\right|_{U_{ \pm} \cap \pi^{-1}(b)}: U_{ \pm} \cap \pi^{-1}(b) \rightarrow D$ are holomorphic coordinates around $p_{ \pm} \in \pi^{-1}(b)$.

Definition 12.1. We define the glueing isomorphism

$$
I_{c}=I_{c}(\boldsymbol{\Sigma}, \lambda): \bigoplus_{\mu \in P_{\ell}} V_{\ell}^{\mathfrak{g}}\left(\boldsymbol{\Sigma}, \mu, \mu^{\dagger}, \lambda\right) \rightarrow V_{\ell}^{\mathfrak{g}}\left(\boldsymbol{\Sigma}_{c}, \lambda\right)
$$

to be equal to $I_{c}\left(\mathbf{C}, x_{ \pm}\right)$for any pair $\left(\mathbf{C}, x_{ \pm}\right)$of a complex structure and holomorphic coordinates on $\left(\Sigma,\left\{p_{+}, p_{-}\right\} \cup P\right)$.

Recall that it is assumed that $\left(\Sigma_{c}, P\right)$ is stable and saturated. Let now $\left(\boldsymbol{\Sigma}^{\prime}, \lambda^{\prime}\right)$ be a labeled marked surface obtained from $(\boldsymbol{\Sigma}, \lambda)$ by labeling further points by $0 \in P_{\ell}$.

Proposition 12.2. We get the following commutative diagram of isomorphisms:

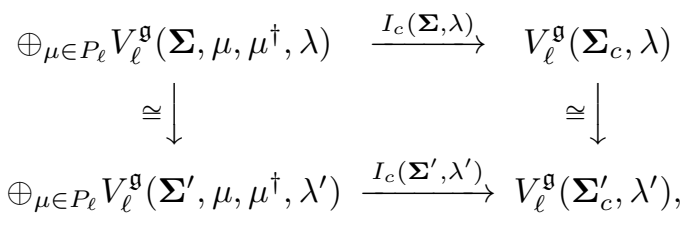

where the vertical isomorphisms are the ones gotten from Theorem 11.1.

Proof. This follows since the glueing constructions both in the non-abelian case and the in the abelian case commutes with the propagation of vacua isomorphism. This is clear from (800) and (82). 
Definition 12.2. In the cases where $(\boldsymbol{\Sigma}, \lambda)$ is not stable or not saturated, we define the glueing isomorphism, to be the unique isomorphism

$$
I_{c}=I_{c}(\boldsymbol{\Sigma}, \lambda): \oplus_{\mu \in P_{\ell}} V_{\ell}^{\mathfrak{g}}\left(\boldsymbol{\Sigma}, \mu, \mu^{\dagger}, \lambda\right) \rightarrow V_{\ell}^{\mathfrak{g}}\left(\boldsymbol{\Sigma}_{c}, \lambda\right)
$$

which makes the diagram (77) commutative for any stable and saturated labeled marked surface $\left(\boldsymbol{\Sigma}_{c}^{\prime}, \lambda^{\prime}\right)$ obtained from $(\boldsymbol{\Sigma}, \lambda)$ by labeling further points by $0 \in P_{\ell}$.

By the naturality of the glueing construction we have that

Proposition 12.3. The glueing isomorphism are compatible with the isomorphisms induced by glueing morphisms of marked surfaces. That is suppose

$$
\mathbf{f}:\left(\Sigma^{1},\left\{p_{-}^{1}, p_{+}^{1}\right\} \sqcup P^{1},\left\{v_{-}^{1}, v_{+}^{1}\right\} \sqcup V^{1}, L^{1}\right) \rightarrow\left(\Sigma^{2},\left\{p_{-}^{2}, p_{+}^{2}\right\} \sqcup P^{2},\left\{v_{-}^{2}, v_{+}^{2}\right\} \sqcup V^{2}, L^{2}\right)
$$

is a morphism of marked surfaces and that there are glueing maps

$$
c_{i}: P\left(T_{p_{-}^{i}} \Sigma^{i}\right) \rightarrow P\left(T_{p_{+}^{i}} \Sigma^{i}\right)
$$

such that $\left(d_{p_{+}^{1}} f\right)^{-1} c_{2} d_{p_{-}^{1}} f=c_{1}$, then we get the following commutative diagram

$$
\begin{array}{cc}
\oplus_{\mu \in P_{\ell}} V_{\ell}^{\mathfrak{g}}\left(\boldsymbol{\Sigma}^{1}, \mu, \mu^{\dagger}, \lambda^{1}\right) \stackrel{I_{c_{1}}\left(\boldsymbol{\Sigma}^{1}, \lambda^{1}\right)}{\longrightarrow} & V_{\ell}^{\mathfrak{g}}\left(\boldsymbol{\Sigma}_{c_{1}}^{1}, \lambda^{1}\right) \\
V_{\ell}^{\mathfrak{g}}(\mathbf{f}) \downarrow & V_{\ell}^{\mathfrak{g}}(\mathbf{f}) \downarrow \\
\oplus_{\mu \in P_{\ell}} V_{\ell}^{\mathfrak{g}}\left(\boldsymbol{\Sigma}^{2}, \mu, \mu^{\dagger}, \lambda^{2}\right) \stackrel{I_{c_{2}}\left(\boldsymbol{\Sigma}^{2}, \lambda^{2}\right)}{\longrightarrow} & V_{\ell}^{\mathfrak{g}}\left(\boldsymbol{\Sigma}_{c_{2}}^{2}, \lambda^{2}\right),
\end{array}
$$

for all labelings $\lambda^{i}$ of $P^{i}$ compatible with $\mathbf{f}$.

We summarize the results on the glueing construction.

Theorem 12.3. There is an isomorphism $I_{c}$ from $\oplus_{\mu \in P_{\ell}} V_{\ell}^{\mathfrak{g}}\left(\boldsymbol{\Sigma}, \mu, \mu^{\dagger}, \lambda\right)$ to $V_{\ell}^{\mathfrak{g}}\left(\boldsymbol{\Sigma}_{c}, \lambda\right)$ as specified in Definition 12.1] and 12.2, which is independent of the glueing map $c$ in the following sense:

If $c_{i}: P\left(T_{p_{-}} \Sigma\right) \rightarrow P\left(T_{p_{+}} \Sigma\right), i=1,2$, are glueing maps and $f: \boldsymbol{\Sigma}_{c_{1}} \rightarrow \boldsymbol{\Sigma}_{c_{2}}$ is a diffeomorphism as described in Remark [2.3, then we have that

$$
I_{c_{2}}=V_{\ell}^{\mathfrak{g}}(f) I_{c_{1}}
$$

as isomorphisms from $\oplus_{\mu \in P_{\ell}} V_{\ell}^{\mathfrak{g}}\left(\boldsymbol{\Sigma}, \mu, \mu^{\dagger}, \lambda\right)$ to $V_{\ell}^{\mathfrak{g}}\left(\boldsymbol{\Sigma}_{c_{2}}, \lambda\right)$. Moreover the isomorphisms $I_{c}$ are compatible with glueing of morphisms of labeled marked surfaces.

Let $\left(\boldsymbol{\Sigma}^{\prime}, \lambda^{\prime}\right)$ be another labeled marked surface, which is stable and saturated. Let $\boldsymbol{\Sigma}^{\prime \prime}$ be the disjoint union of $\boldsymbol{\Sigma}$ and $\boldsymbol{\Sigma}^{\prime}$. Let $\boldsymbol{\Sigma}_{c}^{\prime \prime}$ be the glueing of $\boldsymbol{\Sigma}^{\prime \prime}$ using the glueing map $c$. Then we clearly have that $\boldsymbol{\Sigma}_{c}^{\prime \prime}=\boldsymbol{\Sigma}_{c} \sqcup \boldsymbol{\Sigma}^{\prime}$. It is trivial to check that 
Proposition 12.4. The glueing isomorphism is compatible with the disjoint union isomorphism, namely the following diagram is commutative

$$
\begin{array}{ccc}
\bigoplus_{\mu \in P_{\ell}} V_{\ell}^{\mathfrak{g}}\left(\boldsymbol{\Sigma}^{\prime \prime}, \mu, \mu^{\dagger}, \lambda, \lambda^{\prime}\right) & \longrightarrow & \bigoplus_{\mu \in P_{\ell}} V_{\ell}^{\mathfrak{g}}\left(\boldsymbol{\Sigma}, \mu, \mu^{\dagger}, \lambda,\right) \otimes V_{\ell}^{\mathfrak{g}}\left(\boldsymbol{\Sigma}^{\prime} \lambda^{\prime}\right) \\
I_{c}\left(\boldsymbol{\Sigma}^{\prime \prime}, \lambda, \lambda^{\prime}\right) \downarrow & I_{c}(\boldsymbol{\Sigma}, \lambda) \otimes \operatorname{Id} \downarrow \\
V_{\ell}^{\mathfrak{g}}\left(\boldsymbol{\Sigma}_{c}^{\prime \prime}, \lambda, \lambda^{\prime}\right) & \longrightarrow & V_{\ell}^{\mathfrak{g}}\left(\boldsymbol{\Sigma}_{c}, \lambda\right) \otimes V_{\ell}^{\mathfrak{g}}\left(\boldsymbol{\Sigma}^{\prime}, \lambda^{\prime}\right) .
\end{array}
$$

Let now $\boldsymbol{\Sigma}=\left(\Sigma,\left\{p_{-}^{(1)}, p_{+}^{(1)}, p_{-}^{(2)}, p_{+}^{(2)}\right\} \sqcup P,\left\{v_{-}^{(1)}, v_{+}^{(1)}, v_{-}^{(2)}, v_{+}^{(2)}\right\} \sqcup V, L\right)$ be a marked surface. Let

$$
c^{(i)}: P\left(T_{p_{-}^{(i)}} \Sigma\right) \rightarrow P\left(T_{p_{+}^{(i)}} \Sigma\right)
$$

be glueing maps and $\Sigma_{c^{(i)}}$ the glueing of $\Sigma$ at the ordered pair $\left(\left(p_{-}^{(i)}, v_{-}^{(i)}\right),\left(p_{+}^{(i)}, v_{+}^{(i)}\right)\right)$ with respect to $c^{(i)}$. Let $\Sigma_{c^{(12)}}$ be the glueing with respect to $c^{(12)}=c^{(1)} \sqcup c^{(2)}$.

Theorem 12.4. The glueing isomorphisms commute, meaning the following diagram is commutative

$$
\begin{array}{ccc}
\bigoplus_{\mu_{1}, \mu_{2} \in P_{\ell}} V_{\ell}^{\mathfrak{g}}\left(\boldsymbol{\Sigma}, \mu_{1}, \mu_{1}^{\dagger}, \mu_{2}, \mu_{2}^{\dagger}, \lambda\right) & \stackrel{\oplus_{\mu_{2} \in P_{\ell} I_{c}(1)}\left(\boldsymbol{\Sigma}, \mu_{2}, \mu_{2}^{\dagger}, \lambda\right)}{\longrightarrow} & \bigoplus_{\mu_{2} \in P_{\ell}} V_{\ell}^{\mathfrak{g}}\left(\boldsymbol{\Sigma}_{c(1)}, \mu_{2}, \mu_{2}^{\dagger}, \lambda\right) \\
\bigoplus_{\mu_{1} \in P_{\ell}} I_{c}(2) & \left(\boldsymbol{\Sigma}, \mu_{1}, \mu_{1}^{\dagger}, \lambda\right) \downarrow & I_{c^{(2)}}\left(\boldsymbol{\Sigma}_{c^{(1)}}, \lambda\right) \downarrow \\
\bigoplus_{\mu_{1} \in P_{\ell}} V_{\ell}^{\mathfrak{g}}\left(\boldsymbol{\Sigma}_{c^{(2)}}, \mu_{1}, \mu_{1}^{\dagger}, \lambda\right) & \stackrel{I_{c^{(1)}}\left(\boldsymbol{\Sigma}_{c^{(2)}}, \lambda\right)}{\longrightarrow} & V_{\ell}^{\mathfrak{g}}\left(\boldsymbol{\Sigma}_{c^{(12)}}, \lambda\right) .
\end{array}
$$

Proof. Choose a complex structure on $\Sigma$ and let $\mathbf{C}$ denote the resulting marked Riemann surface. The complex structure $\mathbf{C}$ gives a point in the Teichmüller space $\mathcal{T}_{\left(\Sigma,\left\{p_{-}^{(1)}, p_{+}^{(1)}, p_{-}^{(2)}, p_{+}^{(2)}\right\} \sqcup P\right)}$. Choose centered coordinates $x_{ \pm}^{(i)}: U_{ \pm} \rightarrow D$ around $p_{ \pm}^{(i)}$ with $d_{p_{ \pm}^{(i)}} x_{ \pm}^{(i)}\left(v_{ \pm}^{(i)}\right)=1$ and such that $c^{(i)}=P\left(d_{p_{+}^{(i)}} x_{+}^{(i)}\right)^{-1} \circ P(\bar{*}) \circ P\left(d_{p_{-}^{(i)}} x_{-}^{(i)}\right): P\left(T_{p_{-}^{(i)}} \Sigma\right) \rightarrow P\left(T_{p_{+}^{(i)}} \Sigma\right)$. The following construction of a smooth 3-dimensional complex manifold $\mathcal{C}$ with a holomorphic map $\pi: \mathcal{C} \rightarrow D \times D$ is the main ingredient in this proof :

Let

$\mathcal{C}_{1}=\left\{\left(z_{1}^{(i)}, w_{2}^{(i)}, \tau^{(1)}, \tau^{(2)}\right) \in\left(\mathbb{C}^{2} \sqcup \mathbb{C}^{2}\right) \times D \times D\left|z^{(i)} w^{(i)}=\tau^{(i)},\right| z^{(i)}|<1,| w^{(i)}|<1,| \tau^{(i)} \mid<1, i=1,2\right\}$ and

$$
\mathcal{C}_{2}=\left\{(y, \tau) \in \Sigma \times D \times D\left|y \in U_{ \pm}^{(i)} \Rightarrow\right| x_{ \pm}^{(i)}(y)|>| \tau^{(i)} \mid\right\}
$$

Let then

$$
\mathcal{C}=\mathcal{C}_{1} \cup_{\phi} \mathcal{C}_{2}
$$

where

$$
\phi:\left(\left(U_{-}^{(i)}-p_{-}^{(i)}\right) \times D \times D \cup\left(U_{+}^{(i)}-p_{+}^{(i)}\right) \times D \times D\right) \cap \mathcal{C}_{2} \rightarrow \mathcal{C}_{1}
$$

is given by

$$
\phi(y, \tau)=\left\{\begin{array}{ll}
\left(x_{-}^{(i)}(y), \tau^{(i)} / x_{-}^{(i)}(y), \tau^{(1)}, \tau^{(2)}\right), & y \in U_{-}^{(i)}-p_{-}^{(i)} \\
\left(\tau^{(i)} / x_{+}^{(i)}(y), x_{+}^{(i)}(y), \tau^{(1)}, \tau^{(2)}\right), & y \in U_{+}^{(i)}-p_{+}^{(i)}
\end{array} .\right.
$$


One easily checks that $\mathcal{C}$ is a smooth complex manifold of dimension 3 and that we have an obvious holomorphic projection map $\pi: \mathcal{C} \rightarrow D \times D$. Choose formal neighbourhoods $\vec{\eta}$ for the points $P$. We thus get a family of stable pointed curves with formal neighbourhoods $\mathfrak{F}_{c^{(12)}}$ over $D \times D$ obtained by applying the glueing construction at the two pairs $\left(p_{-}^{(1)}, p_{+}^{(1)}\right)$ and $\left(p_{-}^{(2)}, p_{+}^{(2)}\right)$. Let $p=p_{1} \times p_{2}$ : $\tilde{D} \times \tilde{D} \rightarrow D^{*} \times D^{*}$, be the projection and let $\tilde{\mathfrak{F}}_{c^{(12)}}=p^{*}\left(\left.\mathfrak{F}_{c^{(12)}}\right|_{D^{*} \times D^{*}}\right)$. We denote the two projections onto the first factor by $p^{(1)}: \tilde{D} \times \tilde{D} \rightarrow \tilde{D}$ and onto the second by $p^{(2)}: \tilde{D} \times \tilde{D} \rightarrow \tilde{D}$.

Further let $\tilde{\mathfrak{F}}_{c^{(1)}}$ be the pull back under $p_{1}$ of the normalization of $\left.\mathfrak{F}_{c^{(12)}}\right|_{D^{*} \times\{0\}}$ at $\left[p_{-}^{(2)}\right]=\left[p_{+}^{(2)}\right]$ and $\tilde{\mathfrak{F}}_{c^{(2)}}$ be the pull back under $p_{2}$ of the normalization of $\left.\mathfrak{F}_{c^{(12)}}\right|_{\{0\} \times D^{*}}$ at $\left[p_{-}^{(1)}\right]=\left[p_{+}^{(1)}\right]$. Let $\mathfrak{X}=\left(\mathbf{C},\left\{p_{-}^{(1)}, p_{+}^{(1)}, p_{-}^{(2)}, p_{+}^{(2)}\right\} \sqcup P,\left\{x_{-}^{(1)}, x_{+}^{(1)}, x_{-}^{(2)}, x_{+}^{(2)}\right\} \sqcup \vec{\eta}\right)$.

The glueing construction in the non-abelian case applied to $\mathfrak{F}_{c^{(1)}}$ (respectively to $\left.\mathfrak{F}_{c^{(2)}}\right)$ and then to $\mathfrak{F}_{c^{(12)}}$ results in two two-variable versions of (80) for any element of $\mathcal{V}_{\mu_{1}, \mu_{1}^{\dagger}, \mu_{2}, \mu_{2}^{\dagger}, \nu}(\mathfrak{X})$. Explicitly for an element $\langle\Psi| \in \mathcal{V}_{\mu_{1}, \mu_{1}^{\dagger}, \mu_{2}, \mu_{2}^{\dagger}, \nu}^{\dagger}(\mathfrak{X})$ we get a section $\left\langle\widehat{\Psi}^{(1)}\right|$ of $_{\mathcal{V}_{\mu_{2}, \mu_{2}, \nu}^{\dagger}}^{\dagger}\left(\mathfrak{F}_{c^{(1)}}\right)$ given by

$$
\left\langle\widehat{\Psi}^{(1)} \mid \Phi^{(1)}\right\rangle=\sum_{d=0}^{\infty}\left\{\sum_{i=1}^{m_{d}}\left\langle\Psi \mid v_{i}^{(1)}(d) \otimes v_{(1)}^{i}(d) \otimes \Phi^{(1)}\right\rangle\right\}\left(\tau^{(1)}\right)^{\Delta_{\mu_{1}}+d},
$$

where $\left\{v_{1}^{(1)}(d), \ldots, v_{m_{d}}^{(1)}(d)\right\}$ is a basis of $\mathcal{H}_{\mu_{1}}(d)$ and $\left\{v_{(1)}^{1}(d), \ldots, v_{(1)}^{m_{d}}(d)\right\}$ is the dual basis of $\mathcal{H}_{\mu_{1}^{\dagger}}(d)$ and $\left|\Phi^{(1)}\right\rangle$ is any section of $\mathcal{H}_{\mu_{2}, \mu_{2}^{\dagger}, \nu}$. The holomorphic section $\left\langle\widehat{\Psi}^{(1)}\right|$ is covariant constant along the fibers of $p_{1}$. Next we construct a holomophic section $\left\langle\widehat{\Psi}^{(12)}\right|$ of $\mathcal{V}_{\nu}^{\dagger}\left(\mathfrak{F}_{c^{(12)}}\right)$ determined by

$$
\left\langle\widehat{\Psi}^{(12)} \mid \Phi\right\rangle=\sum_{e=0}^{\infty}\left\{\sum_{j=1}^{m_{e}}\left\langle\widehat{\Psi}^{(1)} \mid v_{j}^{(2)}(e) \otimes v_{(2)}^{j}(e) \otimes \Phi\right\rangle\right\}\left(\tau^{(2)}\right)^{\Delta_{\mu_{2}}+e}
$$

where $\left\{v_{1}^{(2)}(e), \ldots, v_{m_{e}}^{(2)}(e)\right\}$ is a basis of $\mathcal{H}_{\mu_{2}}(e)$ and $\left\{v_{(2)}^{1}(e), \ldots, v_{(2)}^{m_{e}}(e)\right\}$ is the dual basis of $\mathcal{H}_{\mu_{2}^{\dagger}}(e)$ and $|\Phi\rangle$ is any element in $\mathcal{H}_{\nu}$. This section is covariant constant along the fibers of $p^{(1)}$.

Similarly, starting form $\langle\Psi| \in \mathcal{V}_{\mu_{1}, \mu_{1}^{\dagger}, \mu_{2}, \mu_{2}^{\dagger}, \nu}^{\dagger}(\mathfrak{X})$ we can construct a holomorphic section of $\mathcal{V}_{\mu_{1}, \mu_{1}^{\dagger}, \nu}\left(\mathfrak{F}_{c^{(2)}}\right)$, covariant constant along the fibers of $p_{2}$, which is determined by

$$
\left\langle\widehat{\Psi}^{(2)} \mid \Phi\right\rangle=\sum_{e=0}^{\infty}\left\{\sum_{j=1}^{m_{e}}\left\langle\Psi^{(2)} \mid v_{j}^{(2)}(e) \otimes v_{(2)}^{j}(e) \otimes \Phi\right\rangle\right\}\left(\tau^{(2)}\right)^{\Delta_{\mu_{2}}+e} .
$$

Then, starting form $\left\langle\widehat{\Psi}^{(2)}\right|$ we can construct a holomorphic section $\left\langle\widehat{\Psi}^{(21)}\right|$ of $\mathcal{V}_{\nu}^{\dagger}\left(\mathfrak{F}_{c^{(12)}}\right)$ which is covariant constant along the fibers of $p^{(2)}$, and given by

$$
\left\langle\widehat{\Psi}^{(21)} \mid \Phi\right\rangle=\sum_{d=0}^{\infty}\left\{\sum_{i=1}^{m_{d}}\left\langle\widehat{\Psi}^{(2)} \mid v_{i}^{(1)}(d) \otimes v_{(1)}^{i}(d) \otimes \Phi\right\rangle\right\}\left(\tau^{(1)}\right)^{\Delta_{\mu_{1}}+d}
$$

Now $\left\langle\widehat{\Psi}^{(12)}\right|$ and $\left\langle\widehat{\Psi}^{(21)}\right|$ is given by the same power series

$$
\sum_{d=0}^{\infty} \sum_{i=1}^{m_{d}} \sum_{j=1}^{m_{e}}\left\langle\Psi \mid v_{i}^{(1)}(d) \otimes v_{(1)}^{i}(d) \otimes v_{j}^{(2)}(e) \otimes v_{(2)}^{j}(e) \otimes \Phi\right\rangle\left(\tau^{(1)}\right)^{\Delta_{\mu_{1}}+d}\left(\tau^{(2)}\right)^{\Delta_{\mu_{2}}+e} .
$$


Hence, the two sections $\left\langle\widehat{\Psi}^{(12)}\right|$ and $\left\langle\widehat{\Psi}^{(21)}\right|$ of $\mathcal{V}_{\nu}^{\dagger}\left(\mathfrak{F}_{c^{(12)}}\right)$ coincide, and in fact they are covariant constant.

From this we in particular see that the connection in $\mathcal{V}_{\nu}^{\dagger}\left(\tilde{\mathfrak{F}}_{c^{(12)}}\right)$ is flat. By applying the same argument to the abelian theory, we get that the glueing construction is also independent of the order of the glueing in the abelian case and the connection in $\mathcal{V}_{\mathrm{ab}}^{\dagger}\left(\mathfrak{F}_{c^{(12)}}\right)$ is flat over $D \times D$. The theorem now follows.

\section{VERIFICATION OF THE AXIOMS}

It is now straight forward to check the axioms of a modular functor given the results obtained in the previous sections.

Theorem 13.1. The functor $V_{\ell}^{\mathfrak{g}}$ from the category of labeled marked surfaces to the category finite dimensional vector spaces is a modular functor.

Proof. In order to check axiom $M F 1$, we only need to check that the disjoint union isomorphisms satisfies associativity, but this follows from associativity of the isomorphisms between the corresponding $\mathcal{H}^{\dagger}$ 's and $\mathcal{F}$ 's.

We have that the glueing isomorphism $I_{c}$ from Theorem 12.3 is compatible with

- The disjoint union isomorphisms: Proposition 12.4

- The glueing isomorphisms them self, i.e. the glueing isomorphisms should commute: Theorem 12.4

Hence axiom MF2 is checked.

Axiom MF3 is trivial, since we define $V_{\ell}^{\mathfrak{g}}(\emptyset)=\mathbb{C}$. Axiom MF4 and MF5 follows from Corollary $3.5 .2(1)$ and (2) in [19].

\section{Appendix. Families of Stable Curves And GLUeing}

In order to define the functor $V_{\ell}^{\mathfrak{g}}$, we only need to refer to Riemann Surface and families of such which form smooth complex manifolds. However, in defining the glueing morphisms we also need to consider the so called stable nodal curves. These are one dimensional algebraic sub-varieties (algebraic curves) of complex projective space, such that the singularities are locally analytically isomorphic to a neighbourhood of the origin of $x y=0$. A neighbourhood of a node is obtained by patching together at the origins of two small disks $D_{1}=\left\{x ;|| x \mid<\varepsilon_{1}\right\}$ and $D_{2}=\left\{y ;|| y \mid<\varepsilon_{2}\right\}$. By reversing the process, from a neighbourhood of a node we obtain two disconnected small disks. This process is called normalization or desingularization of the node. Thus, by a normalization of a nodal curve $C$ we obtain a compact Riemann surface $\widetilde{C}$ and holomorphic map $\nu: \widetilde{C} \rightarrow C$ such that 
for a node $P$, the inverse image $\nu^{-1}(P)$ consists of two distinct points $P_{+}$and $P_{-}$. A curve which has only nodes as singularities is called a nodal curve.

14.1. Stable curves. We begin by introducing the notion of stable curves.

Definition 14.1. The data $\mathfrak{X}=\left(C ; Q_{1}, Q_{2}, \ldots, Q_{N}\right)$ consisting of an algebraic curve $C$ and points $Q_{1}, \ldots, Q_{N}$ on $C$ are called an $(N$-)pointed stable curve, if the following conditions are satisfied.

(1) The curve $C$ is a nodal curve.

(2) $Q_{1}, Q_{2}, \ldots, Q_{N}$ are non-singular points of the curve $C$.

(3) If an irreducible component $C_{i}$ is a Riemann sphere $\mathbf{P}^{1}$ (resp. a rational curve with one double point, resp. an elliptic curve), the sum of the number of intersection points of $C_{i}$ and other components and the number of $Q_{j}$ 's on $C_{i}$ is at least three (resp. one, resp. one).

(4) $\operatorname{dim}_{\mathbb{C}} H^{1}\left(C, \mathcal{O}_{C}\right)=g$.

Note that the condition (3) is equivalent to saying that the Euler characteristic of each component of the complement of the points $Q_{j}$ on it and the nodes is negative.

A pointed stable curve with formal neighbourhoods is defined in an analogous way as a pointed Riemann surface with formal neighbourhoods (see Definition 3.7). Also we can define a family of pointed stable curves with formal neighbourhoods.

Definition 14.2. The data $\mathfrak{F}=\left(\pi: \mathcal{C} \rightarrow \mathcal{B} ; s_{1}, s_{2}, \ldots, s_{N} ; \eta_{1}, \eta_{2}, \ldots, \eta_{N}\right)$ is called a family of $(\mathrm{N}$-)pointed stable curves of genus $g$ with formal neighbourhoods, if the following conditions are satisfied.

(1) Both $\mathcal{C}$ and $\mathcal{B}$ are connected complex manifolds, $\pi: \mathcal{C} \rightarrow \mathcal{B}$ is a proper flat holomorphic map and $s_{1}, s_{2}, \ldots, s_{N}$ are holomorphic sections of $\pi$.

(2) For each point $b \in \mathcal{B}$ the data $\left(\mathcal{C}_{b}:=\pi^{-1}(b) ; s_{1}(b), s_{2}(b), \ldots, s_{N}(b)\right)$ is an $N$-pointed stable curve of genus $g$.

(3) For each $j, \eta_{j}$ is an $\mathcal{O}_{\mathcal{B}}$-algebra isomorphism

$$
\eta_{j}: \widehat{\mathcal{O}}_{\mathcal{C} / s_{j}}=\lim _{n \rightarrow \infty} \mathcal{O}_{\mathcal{C}} / I_{j}^{n} \simeq \mathcal{O}_{\mathcal{B}}[[\xi]],
$$

where $I_{j}$ is the defining ideal of $s_{j}(\mathcal{B})$ in $Y$.

The only families of pointed stable curves we need in this paper are all constructed explicitly from families of pointed Riemann surfaces via the glueing process discussed in the begining of section 12 .

14.2. Sheaf of vacua for a family of pointed stable curves. For a family of stable curves with formal neighbourhoods $\mathfrak{F}=(\pi: \mathcal{C} \rightarrow \mathcal{B} ; \vec{s} ; \vec{\eta})$ we can define the sheaf of vacua $\mathcal{V}_{\vec{\lambda}}^{\dagger}(\mathfrak{F})$ and the sheaf of covacua $\mathcal{V}_{\vec{\lambda}}(\mathfrak{F})$ just as in Definition 5.1. If the family contains smooth curves (Riemann surfaces), then we have the connection given by Definition 6.2 on the complement of the locus of nodal curves and the connection has a regular singularity along this locus (see $\S 5.3$ of [19]). 
Using this connection with regular singularities it is shown in [19, that Theorem 5.1 is also valid for a family of stable curves.

Theorem 14.1. For a stable family of stable curves with formal neighbourhoods $\mathfrak{F}=(\pi: \mathcal{C} \rightarrow$ $\mathcal{B} ; \vec{s} ; \vec{\eta})$, the sheaves $\mathcal{V}_{\vec{\lambda}}^{\dagger}(\mathfrak{F})$ and $\mathcal{V}_{\vec{\lambda}}(\mathfrak{F})$ are locally free sheaves of $\mathcal{O}_{\mathcal{B}}$-modules of finite rank over $\mathcal{B}$. They are duals to of each other.

We include this theorem here for completeness. We do not need this result for the constructions in this paper.

14.3. The sheaf of abelian vacua associated to families of pointed stable curves. For a family of stable curves with formal neighbourhoods $\mathfrak{F}=(\pi: \mathcal{C} \rightarrow \mathcal{B} ; \vec{s} ; \vec{\eta})$ we can define the sheaf of abelian vacua $\mathcal{V}_{\mathrm{ab}}^{\dagger}(\mathfrak{F})$ just as in Definition 8.1. We have following result.

Theorem 14.2 ([2, Theorem 5.2]). The abelian vacua construction applied to $\mathfrak{F}$ gives a holomorphic line bundle $\mathcal{V}_{\mathrm{ab}}^{\dagger}(\mathfrak{F})$ over $\mathcal{B}$.

Next let us consider a nodal curve $C$ with node $P$. Let $\widetilde{C}$ be the Riemann surface obtained by resolving the singularity at $P$ and let $\pi: \widetilde{C} \rightarrow C$ be the natural holomorphic mapping. Then $\pi^{-1}(P)$ consists of two points $P_{+}$and $P_{-}$. Let

$$
\mathfrak{X}=\left(C ; q_{1}, \ldots, q_{N} ; \xi_{1}, \ldots, \xi_{N}\right)
$$

be a pointed nodal curve with formal neighbourhoods and we let

$$
\widetilde{\mathfrak{X}}=\left(\widetilde{C} ; P_{+}, P_{-}, q_{1}, \ldots, q_{N} ; z, w, \xi_{1}, \ldots, \xi_{N}\right)
$$

be the associated pointed Riemann surface with formal neighbourhoods. Define an element $\left|0_{+,-}\right\rangle \in$ $\mathcal{F} \otimes \mathcal{F}$ by

$$
\left|0_{+,-}\right\rangle=|0\rangle \otimes|-1\rangle-|-1\rangle \otimes|0\rangle
$$

The natural inclusion

$$
\begin{aligned}
\mathcal{F}_{N} & \hookrightarrow \mathcal{F}_{N+2} \\
|u\rangle & \mapsto\left|0_{+,-}\right\rangle \otimes|u\rangle
\end{aligned}
$$

defines a natural linear mapping

$$
\iota_{+,-}^{*}: \mathcal{F}_{N+2}^{\dagger} \rightarrow \mathcal{F}_{N}^{\dagger}
$$

Theorem 14.3 ([2, Theorem 3.5]). The natural mapping $\iota_{+,-}^{*}$ induces a natural isomorphism

$$
\mathcal{V}_{\mathrm{ab}}^{\dagger}(\widetilde{\mathfrak{X}}) \cong \mathcal{V}_{\mathrm{ab}}^{\dagger}(\mathfrak{X})
$$


Let us now consider the case where we have one marked point on $C$

$$
\mathfrak{X}=(C ; Q ; \xi)
$$

and we let

$$
\widetilde{\mathfrak{X}}=\left(\widetilde{C} ; P_{+}, P_{-}, Q ; z, w, \xi\right)
$$

be the associated 3 -pointed curve with formal neighbourhoods. We can define $\langle\omega(\mathfrak{X},\{\alpha, \beta\})|$ similar to the non-singular case, by choosing a basis

$$
(\vec{\alpha}, \vec{\beta})=\left(\alpha_{1}, \ldots, \alpha_{g-1}, \alpha_{g}, \beta_{1}, \ldots, \beta_{g-1}\right)
$$

of $H_{1}(C, \mathbf{Z})$, in such a way that $\alpha_{1}, \alpha_{2}, \ldots, \alpha_{g-1}$ and $\beta_{1}, \beta_{2}, \ldots, \beta_{g-1}$ is the image of a symplectic basis of $H_{1}(\widetilde{C}, \mathbf{Z})$ under natural map to $H_{1}(C, \mathbf{Z})$ and $\alpha_{g}$ corresponds to the invariant cycle of a flat deformation of the curve $C$. Then we can choose a basis $\left\{\omega_{1}, \ldots, \omega_{g-1}, \omega_{g}, \omega_{g+1}, \omega_{g+2}, \ldots\right\}$ of $H^{0}(C, \omega(* Q))$ such that $\left\{\pi^{*} \omega_{1}, \ldots, \pi^{*} \omega_{g-1}, \pi^{*} \omega_{g+1}, \pi^{*} \omega_{g+2}, \ldots\right\}$ is a normalized basis of $H^{0}\left(\widetilde{C}, \omega_{\widetilde{C}}(* Q)\right)$ as in (69), (170) and (71) where we put

$$
\pi^{*} \omega_{g+n}=\omega_{Q}^{(n)}, \quad n=1,2, \ldots,
$$

and $\pi^{*} \omega_{g}$ is a meromorphic one-form on $\widetilde{C}$ which has poles of order one at $P_{+}$and $P_{-}$with residue -1 and 1 , respectively, is holomorphic outside $P_{ \pm}$and

$$
\int_{P_{+}}^{P_{-}} \pi^{*} \omega_{g}=1 .
$$

Then put

$$
\langle\omega(\mathfrak{X},\{\alpha, \beta\})|=\left\langle\cdots \wedge e\left(\omega_{m}\right) \wedge \cdots \wedge e\left(\omega_{2}\right) \wedge e\left(\omega_{1}\right)\right| .
$$

The proof of Lemma 3.1 of [2] applies also in this case and shows that $\langle\omega(\mathfrak{X},\{\alpha, \beta\})|$ is an element of $\mathcal{V}_{\mathrm{ab}}^{\dagger}(\mathfrak{X})$. Let

$$
\widehat{\mathfrak{X}}=(\widetilde{C}, Q ; \xi) .
$$

Then, by applying Theorem 7.4 at the points $P_{ \pm}$we have a canonical isomorphism

$$
\iota^{*}: \mathcal{V}_{\mathrm{ab}}^{\dagger}(\widetilde{\mathfrak{X}}) \cong \mathcal{V}_{\mathrm{ab}}^{\dagger}(\widehat{\mathfrak{X}})
$$

Theorem 14.4 ([2, Theorem 6.5]). Under the above assumptions and notation we have that

$$
\iota^{*} \circ\left(\iota_{+,-}^{*}\right)^{-1}(\langle\omega(\mathfrak{X},\{\alpha, \beta\})|)=(-1)^{g}\langle\omega(\widehat{\mathfrak{X}},\{\widehat{\alpha}, \widehat{\beta}\})|
$$

where $\{\widehat{\alpha}, \widehat{\beta}\}=\left\{\alpha_{1}, \ldots, \alpha_{g-1}, \beta_{1}, \ldots, \beta_{g-1}\right\}$.

Let $\mathfrak{F}=\left(\pi: \mathcal{C} \rightarrow \mathcal{B}, s_{1}, \ldots, s_{N}, \xi_{1}, \ldots, \xi_{N}\right)$ be a family of $N$-pointed Riemann surfaces. For any point $b \in \mathcal{B}$ there exists an open neighbourhood $U_{b}$ such that $\pi^{-1}\left(U_{b}\right)$ is topologically trivial so that we can choose smoothly varying symplectic bases

$$
\left(\alpha_{1}(t), \ldots, \alpha_{g}(t), \beta_{1}(t), \ldots, \beta_{g}(t)\right), \quad t \in \mathcal{B} .
$$


Then we can define, using propagation of vacua,

$$
\left\langle\omega\left(\mathfrak{X}_{t},\{\alpha(t), \beta(t)\}\right)\right| \in \mathcal{V}_{\mathrm{ab}}^{\dagger}(\mathfrak{F})_{t}
$$

where $\mathfrak{X}_{t}=\left(\pi^{-1}(t), s_{1}(t), \xi_{1}\right)$.

Theorem 14.5 ([2, Theorem 6.6]). The section $\left\langle\omega\left(\mathfrak{X}_{t},\{\alpha(t), \beta(t)\}\right)\right|$ is a holomorphic section of $\mathcal{V}_{\mathrm{ab}}^{\dagger}(\mathfrak{F})$ over $U_{b}$.

14.4. The glueing of vacua construction. Let us briefly recall the glueing of vacua. First let us consider the non-abelian case. We use freely the notation in $₫ 12$. Let $\mathfrak{F}=\left(\pi: \mathcal{C} \rightarrow \mathcal{B} ; s_{-}, s_{+}, \vec{s} ; \eta_{-}, \eta_{+}, \vec{\eta}\right)$ be a family of pointed Riemann surfaces with formal neighbourhoods on $\boldsymbol{\Sigma}$ over a simply-connected base $\mathcal{B}$ and we let $\mathfrak{F}_{c}=\left(\pi_{c}: \mathcal{C}_{c} \rightarrow \mathcal{B}_{c}, \vec{s}, \vec{\eta}\right)$ be a stable and saturated family of $N$-pointed curves with formal neighbourhoods obtained by applying the glueing construction pointwise over $\mathcal{B}$ to $\mathfrak{F}$ where $\mathcal{B}_{c}=\mathcal{B} \times D$ with the unit disk $D$.

By the isomorphism (75) holomorphic sections of $\oplus_{\mu} \mathcal{V}_{\mu, \mu^{\dagger}, \vec{\lambda}}^{\dagger}(\mathfrak{F})$ over $\mathcal{B}$ may be regarded as holomorphic sections of $\mathcal{V}_{\vec{\lambda}}^{\dagger}\left(\left.\mathfrak{F}_{c}\right|_{\mathcal{B} \times\{0\}}\right)$ over $\mathcal{B} \times\{0\}$. The glueing of vacua is an isomorphism from holomorphic section of $\oplus_{\mu} \mathcal{V}_{\mu, \mu^{\dagger}, \vec{\lambda}}^{\dagger}(\mathfrak{F})$ over $\mathcal{B}$ to a holomorphic sections of $\mathcal{V}_{\vec{\lambda}}^{\dagger}\left(\tilde{\mathfrak{F}}_{c}\right)$ over $\tilde{\mathcal{B}}_{c}$, which is covariant constant along the direction of $\tilde{D}$. The construction is as follows.

By Lemma 4.1 we can choose a basis $\left\{v_{1}(d), \ldots, v_{m_{d}}(d)\right\}$ of $\mathcal{H}_{\mu}(d)$ and the dual basis $\left\{v^{1}(d), \ldots, v^{m_{d}}(d)\right\}$ of $\mathcal{H}_{\mu^{\dagger}}(d)$ such that

$$
\left(v^{j}(d) \mid v_{k}(d)\right)=\delta_{k}^{j} .
$$

For a holomorphic section $\langle\Psi| \in \mathcal{V}_{\mu, \mu^{\dagger}, \vec{\lambda}}^{\dagger}(\mathfrak{F})$ over $\mathcal{B}$ we define a formal series $\langle\widehat{\Psi}|$ by

$$
\langle\widehat{\Psi} \mid \Phi\rangle=\sum_{d=0}^{\infty}\left\{\sum_{i=1}^{m_{d}}\left\langle\Psi \mid v_{i}(d) \otimes v^{i}(d) \otimes \Phi\right\rangle\right\} \tau^{\Delta_{\mu}+d},
$$

for all $|\Phi\rangle \in \mathcal{H}_{\vec{\lambda}}$. Here the fractional power $\tau^{\Delta_{\mu}+d}$ is clearly well defined on $\tilde{D}$. This formal power series converges and defines in fact a holomorphic section of $\mathcal{V}_{\vec{\lambda}}^{\dagger}\left(\mathfrak{F}_{c}\right)$. Namely, we have the following theorem.

Theorem 14.6 ([19, Theorem 5.3.4]). The formal power series $\langle\widehat{\Psi}|$ is a formal solution of the differential equation

$$
\left(\tau \frac{d}{d \tau}-T[\vec{l}]+a(\vec{l})\right)\langle\widehat{\Psi}|=0
$$

of Fuchsian type where

$$
\vec{l}=\left(l_{1}\left(\xi_{1}\right) \frac{d}{d \xi_{1}}, \ldots, l_{N}\left(\xi_{N}\right) \frac{d}{d \xi_{N}}\right)
$$

is an $N$-tuple of formal vector fields such that $\theta(l)=\tau \frac{d}{d \tau}$. Moreover, $\langle\widehat{\Psi}|$ converges and defines a holomorphic section of $\mathcal{V}_{\vec{\lambda}}^{\dagger}\left(\tilde{\mathfrak{F}}_{c}\right)$ over $\tilde{\mathcal{B}}_{c}$. 
The differential equation (81) gives the holomorphic connection along the direction of $\tilde{D}$. Hence, the above formal power series is a holomorphic section of $\mathcal{V}_{\vec{\lambda}}^{\dagger}\left(\mathfrak{F}_{c}\right)$ which is covariant constant along the direction of $\tilde{D}$.

Let us now discuss the glueing in the abelain case.

Recall we have a perfect pairing

$$
\{\mid\}_{+}: \mathcal{F}_{d}(p) \times \mathcal{F}_{d}(-p-1) .
$$

Let $\left\{v_{i}(d, p)\right\}_{i=1, \ldots, m_{d}}$ be a basis of $\mathcal{F}_{d}(p)$ for any $p \in \mathbf{Z}$ and $\left\{v^{i}(d, p)\right\}_{i=1, \ldots, m_{d}}$ be the dual basis of $\mathcal{F}_{d}(-p-1)$ with respect to the pairing $\{\mid\}_{+}$.

For a holomorphic section $\langle\psi|$ of $\mathcal{V}_{\mathrm{ab}}^{\dagger}(\mathfrak{F})$ define $\langle\widetilde{\psi}|$ by

$$
\langle\widetilde{\psi} \mid u\rangle=\sum_{p \in \mathbf{Z}}\left\{\sum_{d=0}^{\infty} \sum_{i=1}^{m_{d}}(-1)^{p+d}\left\langle\psi \mid v_{i}(d, p) \otimes v^{i}(d,-p-1) \otimes u\right\rangle\right\} \tau^{d+p(p+1) / 2} .
$$

Then the formal power series converges and defines a holomorphic section of $\langle\widetilde{\psi}| \in \mathcal{V}_{\mathrm{ab}}^{\dagger}\left(\mathfrak{F}_{c}\right)$. Moreover this section is covariant constant along the directions of $D$.

Theorem 14.7. The glueing construction gives an isomorphism between sections of $\mathcal{V}_{\mathrm{ab}}^{\dagger}(\mathfrak{F})$ and sections of $\mathcal{V}_{\mathrm{ab}}^{\dagger}\left(\mathfrak{F}_{c}\right)$ which are covariant constant along the directions of $D$.

This theorem follows directly form Theorem 5.3 in [2]. Finally, we analyze the preferred section for the families. Suppose we have a continuous basis $\left(\alpha_{i}(t), \beta_{i}(t)\right)$ of $H_{1}\left(\pi^{-1}(t), \mathbf{Z}\right), t \in(0,1) \subset$ $D$, such that we get a well defined limit as $t$ goes to zero, which gives a symplectic basis, say $\left(\alpha_{1}(0), \ldots, \alpha_{g-1}(0), \alpha_{g}(0), \beta_{1}(0), \ldots, \beta_{g-1}(0)\right)$ of $H_{1}\left(C_{0}, \mathbf{Z}\right)$ as described above for nodal curves and $\beta_{g}(0)=0$. Let $\mathfrak{X}_{t}=\left(\pi^{-1}(t), s_{1}(t), \ldots s_{N}(t), \xi_{1}, \ldots \xi_{N}\right)$.

Theorem 14.8 ([2, Theorem 6.7]). We have that

$$
\left\langle\omega\left(\mathfrak{X}_{0},\{\alpha(0), \beta(0)\}\right)\right|=\lim _{t \rightarrow 0}\left\langle\omega\left(\mathfrak{X}_{t},\{\alpha(t), \beta(t)\}\right)\right| .
$$

\section{REFERENCES}

[1] J. E. Andersen, The Witten invariant of finite oder mapping tori I, To appear in Journal für Reine und Angewandte Matematik.

[2] J. E. Andersen \& K. Ueno, Abelian Conformal Field Theory and Determinant Bundles, MPS-Preprint 2003 - 5, math.QA/0304135

[3] J. E. Andersen \& K. Ueno, Construction of the Reshetikhin-Turaev TQFT from conformal field theory, Preprint in preparation.

[4] M.F. Atiyah, On Framings of 3-manifolds, Topology 29 (1990) 1-7

[5] B. Bakalov; A. Jr. Kirillov, Lectures on tensor categories and modular functors, University Lecture Series, 21. American Mathematical Society, Providence, RI, 2001.

[6] C. Blanchet, Hecke algebras, modular categories and 3-manifolds quantum invariants, Topology 39 no. 1, 193-223, 2000. 
[7] C. Blanchet; N. Habegger; G. Masbaum; P. Vogel, Three-manifold invariants derived from the Kauffman bracket, Topology 31 no. 4, 685-699, 1992.

[8] C. Blanchet; N. Habegger; G. Masbaum; P. Vogel, Topological quantum field theories derived from the Kauffman bracket, Topology 34 no. 4, 883-927, 1995.

[9] J. D. Fay, Theta functions on Riemann surfaces, Lecture Notes in Math., 352, Springer-Verlag, 1973.

[10] G. Felder, Brst approach to minimal models, Nucl. Phys. B, 317, 215-236, 1989.

[11] J. Grove, Constructing TQFTs from modular functors, J. Knot Theory Ramifications 10 no. 8, 1085-1131, 2001.

[12] V. Kac, Infinite dimensional Lie algebras, third edition, Cambridge University Press, 1990.

[13] N. Kawamoto, Y. Namikawa, A. Tsuchiya and Y. Yamada, Geometric realization of conformal field theory on Riemann surfaces, Comm. Math. Phys., 116, 247-308, 1988.

[14] M. Kontsevich, Rational conformal field theory and invariants of 3-manifolds, Preprint of Centre de Physique Theorique Marseille, CPT-88/p2189, 1988.

[15] G. Segal, The definition of conformal field theory, preprint 1992.

[16] A. Tsuchiya, K. Ueno and Y. Yamada", Conformal Field Theory on Universal Family of Stable Curves with Gauge Symmetries, Advanced Studies in Pure Mathematics 19, 459-566, 1989.

[17] V. Turaev, Quantum invariants of knots and 3-manifolds, W. de Gruyter, Berlin, 1994..

[18] K. Ueno, On conformal field theory, London Math. Soc. Lecture Note 208, 283-345, 1995.

[19] K. Ueno, Introduction to conformal field theory with gauge symmetries, Geometry and physics (Aarhus, 1995), Lecture Notes in Pure and Appl. Math. 184, 603-745, Dekker, New York, 1997.

[20] K. Walker, On Witten's 3-manifold invariants, Preliminary version \# 2, Preprint 1991.

[21] C.T.C. Wall, Non-additivity of the signature, Invent. Math. 7 (1969) 269-274

Department of Mathematics, University of Aarhus, DK-8000, Denmark

E-mail address: andersen@imf.au.dk

Department of Mathematics, Faculty of Science, Kyoto University, Kyoto, 606-01 Japan

E-mail address: ueno@math.kyoto-u.ac.jp 